Supporting Information

\title{
"Clickphine": a novel and highly versatile P,N ligand class via click chemistry.
}

\author{
Remko J. Detz, Silvia Arévalo Heras, R. de Gelder, ${ }^{\dagger}$ Piet W. N. M. van Leeuwen, \\ Henk Hiemstra, Joost N. H. Reek* and Jan H. van Maarseveen*
}

Van 't Hoff Institute for Molecular Sciences, Faculty of Science, University of Amsterdam, Nieuwe Achtergracht 129, 1018 WS Amsterdam, The Netherlands; ${ }^{\dagger}$ Molecular Materials, Institute for Molecules and Materials, Radboud University Nijmegen, Toernooiveld 1, 6525 ED Nijmegen, The Netherlands.

E-mail: jvm@science.uva.nl; reek@science.uva.nl

General Methods. The following general procedures were used in all reactions unless noted otherwise. Oxygen- and moisture-sensitive reactions were carried out using standard Schlenk techniques under a nitrogen or argon atmosphere. Air sensitive liquids and solutions were transferred via a gas-tight syringe or cannula. Reactions were stirred with a Teflon-covered magnetic stirring bar. Removal of solvents was accomplished by evaporation on a Buchi rotary evaporator (water bath $40{ }^{\circ} \mathrm{C}$ ) or directly from the schlenk flask using an oil pump. Tetrahydrofuran and diethyl ether were freshly distilled from sodium/benzophenone. Toluene was distilled from sodium. Dry $\mathrm{CH}_{2} \mathrm{Cl}_{2}$ was freshly distilled from $\mathrm{CaH}_{2}$. All commercially available reagents were used as received, unless indicated otherwise.

Chromatography. TLC was performed using $250 \mu \mathrm{m}$ silica gel 60 plates with 254 $\mathrm{nm}$ fluorescent indicator. Chromatographic purification refers to flash chromatography ${ }^{1}$ using the indicated solvent (mixture) and Biosolve silica gel (0.035$0.070 \mathrm{~mm}$ ). Analytical HPLC was carried out on reversed phase $\mathrm{C}_{18}$ columns using gradients between $95: 5: 0.01$ (water / acetonitrile / formic acid) and $5: 95: 0.01$.

Physical and Spectroscopic Measurements. NMR spectra were recorded in Fourier Transform mode on a Varian Mercury VX $\left({ }^{1} \mathrm{H}\right.$ at $300 \mathrm{MHz},{ }^{13} \mathrm{C}$ at $75 \mathrm{MHz},{ }^{31} \mathrm{P}$ at $121 \mathrm{MHz})$ or a Bruker AV $400\left({ }^{1} \mathrm{H}\right.$ at $400 \mathrm{MHz},{ }^{13} \mathrm{C}$ at $101 \mathrm{MHz},{ }^{31} \mathrm{P}$ at $\left.162 \mathrm{MHz}\right)$ or a Varian Unity Inova $\left({ }^{1} \mathrm{H}\right.$ at $500 \mathrm{MHz},{ }^{13} \mathrm{C}$ at $126 \mathrm{MHz},{ }^{31} \mathrm{P}$ at $\left.203 \mathrm{MHz}\right)$ magnetic resonance spectrometer at $25{ }^{\circ} \mathrm{C}$. ${ }^{31} \mathrm{P}$ NMR spectra of resins were recorded on the Varian Unity Inova using a Nano-probe $(1 \mathrm{H}\{31 \mathrm{P}\} 4 \mathrm{~mm}$ PFG Indirect Detection Nano 
Probe) and Magic angle spinning (MAS) techniques with a spin rate of $3000 \mathrm{~Hz}$. The Nano-probe contains a suspension of the resin beads in $d_{2}$-dichloromethane $(40 \mu l)$. NMR spectra are reported as chemical shifts in parts per million (ppm) relative to the solvent signal and converted to tetramethylsilane scale $\left(\mathrm{CDCl}_{3}:{ }^{1} \mathrm{H}, 7.26 \mathrm{ppm} ;{ }^{13} \mathrm{C}\right.$, $77.16 \mathrm{ppm}) .{ }^{31} \mathrm{P}$ NMR spectra were calibrated using $85 \% \mathrm{H}_{3} \mathrm{PO}_{4}$ as an external chemical shift reference. Spin multiplicity is described by the following abbreviations: $\mathrm{s}=$ singlet, $\mathrm{d}=$ doublet, $\mathrm{t}=$ triplet, $\mathrm{q}=$ quartet, $\mathrm{m}=$ multiplet, $\mathrm{dd}=$ doublet of doublets, and $\mathrm{br}=$ broad. Coupling constants $(\mathcal{J})$ are reported in Hertz $(\mathrm{Hz}) .{ }^{13} \mathrm{C}$ NMR spectra were recorded with protondecoupling as APT (attached proton test) spectra. Infrared spectra were obtained from $\mathrm{CDCl}_{3}$ solutions or from solid in $\mathrm{KBr}$ on a Bruker IFS 28 Fourier Transform spectrometer (FTIR) and are reported in wavenumbers $\left(\mathrm{cm}^{-1}\right)$. Fast Atom Bombardment (FAB) mass spectrometry was carried out using a JEOL JMS SX/SX 102A four-sector mass spectrometer, coupled to a JEOL MSMP9021D/UPD system program. Samples were loaded in a matrix solution (3nitrobenzyl alcohol) on a stainless steel probe and bombarded with xenon atoms with an energy of $3 \mathrm{keV}$. During the high resolution FAB-MS measurements a resolving power of 10,000 (10\% valley definition) was used.

Abbreviations. BuLi, butyllithium; $\mathrm{BuOH}$, butanol; DABCO, 1,4diazabicyclo[2.2.2]octane; DCM, dichloromethane; DIPEA, diisopropylethylamine; DMSO, dimethylsulfoxide; EtOAc, ethylacetate; $\mathrm{MeOH}$, methanol; PE, petroleum ether; PS, polystyrene; TBTA, tris-(benzyltriazolylmethyl)amine; Tf, trifluoromethylsulfonyl; THF, tetrahydrofuran.<smiles>[B]P(CC#C)c1ccccc1</smiles>

Propargyl(diphenyl)phosphine borane complex (2). To a solution of the commercially available (diphenyl)phosphine borane complex 1 (1.00 g, 5.00 $\mathrm{mmol})$ in THF (19 mL) $n$-BuLi (1.6 M in hexane, $3.13 \mathrm{~mL}, 5.00 \mathrm{mmol})$ was added at $72{ }^{\circ} \mathrm{C}$ under nitrogen atmosphere. The solution was stirred for $15 \mathrm{~min}$ and propargylbromide ( $80 \%$ in toluene, $0.61 \mathrm{~mL}, 5.5 \mathrm{mmol}$ ) was added, quenching the phosphine anion at $-72{ }^{\circ} \mathrm{C}$. The reaction was finishe d almost directly and after 15 minutes water was added and the solution was warmed to room temperature. The water layer $(100 \mathrm{~mL})$ was extracted with EtOAc $(3 \times 50 \mathrm{~mL})$ and the combined organic layers were washed with water $(80 \mathrm{~mL})$ and brine $(80 \mathrm{~mL})$. The organic phase was dried with anhydrous $\mathrm{Na}_{2} \mathrm{SO}_{4}$. Evaporation of the solvent gave product 2 $(1.17 \mathrm{~g}, 99 \%)$ as a pale yellow oil, which solidified at $-18{ }^{\circ} \mathrm{C} .{ }^{1} \mathrm{H} \mathrm{NMR}(400 \mathrm{MHz}) ; \delta$ 
$(\mathrm{ppm})=7.78-7.73(\mathrm{~m}, 4 \mathrm{H}$ of $m-\mathrm{Ph}), 7.55-7.45(\mathrm{~m}, 6 \mathrm{H}$ of $o, p-\mathrm{Ph}), 3.14(\mathrm{dd}, J=2.8 \mathrm{~Hz}$, $\left.J_{\mathrm{HP}}=10.4 \mathrm{~Hz}, 2 \mathrm{H}, \mathrm{CH}_{2}\right), 2.08(\mathrm{~m}, 1 \mathrm{H}$, acetylene- $\mathrm{H}), 1.5-0.5\left(\mathrm{br}, 3 \mathrm{H}, \mathrm{BH}_{3}\right) ;{ }^{13} \mathrm{C} \mathrm{NMR}$ $(101 \mathrm{MHz}) ; \delta(\mathrm{ppm})=132.7(\mathrm{~d}, J=9.5 \mathrm{~Hz}), 131.8(\mathrm{~d}, J=2.5 \mathrm{~Hz}), 128.9(\mathrm{~d}, J=10.2$ Hz), 127.9 (d, $J=55.5 \mathrm{~Hz}$ ), 75.8 (d, $J=10.5 \mathrm{~Hz}$ ), 73.0 (d, $J=6.6 \mathrm{~Hz}), 18.6$ (d, $J=$ $35.4 \mathrm{~Hz}) ;{ }^{31} \mathrm{P}$ NMR $(162 \mathrm{MHz}) ; \delta(\mathrm{ppm})=18.6(\mathrm{~d}, J=63.2 \mathrm{~Hz})$; FTIR (film, $\mathrm{cm}^{-1}$ ); 3291 (s), 2383 (s), 1437 (s), 1107 (m), 1059 (s); HRMS (FAB+) m/z: calcd. (M+H) 237.1004, found 237.1014.<smiles>FC(F)(F)c1ccc(-n2cc(CP)nn2)cc1</smiles>

4-((diphenyl(borane)phosphino)methyl)-1-(4(trifluoromethyl)phenyl)-1H-1,2,3-triazole (3a). To a mixture of $2(0.10 \mathrm{~g}, 0.42$ $\mathrm{mmol})$ and 4-trifluoromethylphenylazide (79 mg, $0.42 \mathrm{mmol})$ in $t$ - $\mathrm{BuOH}(2 \mathrm{~mL}) \mathrm{a}$ solution of $\mathrm{CuSO}_{4} \bullet 5 \mathrm{H}_{2} \mathrm{O}(1.0 \mathrm{mg}, 0.004 \mathrm{mmol})$ and sodium ascorbate $(8.3 \mathrm{mg}, 0.042$ $\mathrm{mmol}$ ) in $1 \mathrm{~mL}$ water was added. This mixture was stirred at room temperature for 22 hours under nitrogen atmosphere. After addition of $10 \mathrm{~mL}$ water the aqueous phase was extracted with EtOAc $(3 \times 8 \mathrm{~mL})$ and the combined organic layers were washed with water $(7 \mathrm{~mL})$ and brine $(7 \mathrm{~mL})$. The organic phase was dried with anhydrous $\mathrm{Na}_{2} \mathrm{SO}_{4}$. Product 3a (160 mg, 90\%) was obtained after evaporation of the solvent as a white solid. Recrystallisation from EtOAc gave in two combined portions the product (157 mg, 88\%) as white needles: $\mathrm{mp} 159-163{ }^{\circ} \mathrm{C} .{ }^{1} \mathrm{H}$ NMR $(400 \mathrm{MHz}) ; \delta$ $(\mathrm{ppm})=8.02(\mathrm{~d}, J=2.1 \mathrm{~Hz}, 1 \mathrm{H}$, triazole-H), 7.83-7.72 $(\mathrm{m}, 8 \mathrm{H}), 7.53-7.43(\mathrm{~m}, 6 \mathrm{H})$, $3.87\left(\mathrm{~d}, \mathrm{~J}_{\mathrm{HP}}=11.3 \mathrm{~Hz}, 2 \mathrm{H}, \mathrm{CH}_{2}\right), 1.6-0.6\left(\mathrm{br}, 3 \mathrm{H}, \mathrm{BH}_{3}\right) ;{ }^{13} \mathrm{C} \mathrm{NMR}(101 \mathrm{MHz}) ; \delta(\mathrm{ppm})$ $=140.3(\mathrm{~d}, J=2.1 \mathrm{~Hz}), 139.4,132.5(\mathrm{~d}, J=9.3 \mathrm{~Hz}), 131.8(\mathrm{~d}, J=2.3 \mathrm{~Hz}), 130.8(\mathrm{q}, J$ = 33.2 Hz), 129.1 (d, $J=10.0 \mathrm{~Hz}), 128.2(\mathrm{~d}, J=55.4 \mathrm{~Hz}), 127.2(\mathrm{q}, J=3.7 \mathrm{~Hz}$ ), 123.6 (q, $J=272.5 \mathrm{~Hz}$ ), 121.4 (d, $J=2.9 \mathrm{~Hz}), 120.5,24.7$ (d, $J=36.3 \mathrm{~Hz}$ ); ${ }^{31} \mathrm{P}$ NMR $(162 \mathrm{MHz}) ; \delta(\mathrm{ppm})=16.4(\mathrm{~d}, J=51.8 \mathrm{~Hz}) ;$ FTIR (film, $\left.\mathrm{cm}^{-1}\right) ; 2385(\mathrm{~s}), 1616(\mathrm{w})$, 1441 (w), 1326 (s), $1171(\mathrm{~m}), 1131$ (s), 1067 (s), $845(\mathrm{~m})$.

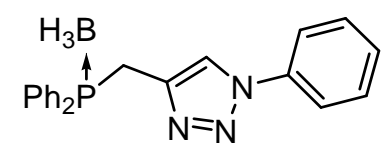

\section{4-((diphenyl(borane)phosphino)methyl)-1-phenyl-1 H-}

1,2,3-triazole (3b). To a mixture of $2(1.19 \mathrm{~g}, 5.00 \mathrm{mmol})$ and phenylazide $(0.55 \mathrm{~mL}$, $5.0 \mathrm{mmol})$ in $t$ - $\mathrm{BuOH}(10 \mathrm{~mL})$ and water $(8 \mathrm{~mL})$ a solution of $\mathrm{CuSO}_{4} \bullet 5 \mathrm{H}_{2} \mathrm{O}(12.5 \mathrm{mg}$, $0.050 \mathrm{mmol}$ ) in $1 \mathrm{~mL}$ water and a sodium ascorbate $(99 \mathrm{mg}, 0.50 \mathrm{mmol}$ ) solution in 1 $\mathrm{mL}$ water were added. This mixture was stirred at room temperature for 2 hours. 
After addition of $90 \mathrm{~mL}$ water the aqueous phase was extracted with EtOAc $(3 \times 40$ $\mathrm{mL})$ and the combined organic layers were washed with water $(60 \mathrm{~mL})$ and brine $(60$ $\mathrm{mL}$ ). The organic phase was dried with anhydrous $\mathrm{Na}_{2} \mathrm{SO}_{4}$. Product $\mathbf{3 b}(1.72 \mathrm{~g}, 96 \%)$ was obtained after evaporation of the solvent as a solid. Recrystallisation from EtOAc with some drops of PE gave the product $(1.33 \mathrm{~g}, 75 \%)$ as light yellow needles: $\mathrm{mp}$ 125-130 $\mathrm{C}$. ${ }^{1} \mathrm{H}$ NMR $(400 \mathrm{MHz}) ; \delta(\mathrm{ppm})=7.92(\mathrm{~d}, J=1.7 \mathrm{~Hz}, 1 \mathrm{H}$, triazole-H), 7.76$7.72(\mathrm{~m}, 4 \mathrm{H}, m-\mathrm{Ph}-\mathrm{P}), 7.64$ (d, $J=8.0 \mathrm{~Hz}, 2 \mathrm{H}, m$-Ph-triazole), 7.50-7.41 (m, 8H, o,p$\mathrm{Ph}), 3.86\left(\mathrm{~d}, \mathrm{~J}_{\mathrm{HP}}=11.1 \mathrm{~Hz}, 2 \mathrm{H}, \mathrm{CH}_{2}\right), 1.5-0.5\left(\mathrm{br}, 3 \mathrm{H}, \mathrm{BH}_{3}\right) ;{ }^{13} \mathrm{C} \mathrm{NMR}(101 \mathrm{MHz}) ; \delta$ $(\mathrm{ppm})=139.7,136.9,132.4(\mathrm{~d}, J=9.3 \mathrm{~Hz}), 131.6(\mathrm{~d}, J=2.3 \mathrm{~Hz}), 129.7,128.9(\mathrm{~d}, J$ $=10.1 \mathrm{~Hz}), 128.7,128.2(\mathrm{~d}, J=55.4 \mathrm{~Hz}), 121.4,120.4,24.6(\mathrm{~d}, J=36.2 \mathrm{~Hz}) ;{ }^{31} \mathrm{P}$ $\operatorname{NMR}(162 \mathrm{MHz}) ; \delta(\mathrm{ppm})=16.3(\mathrm{~d}, J=63.2 \mathrm{~Hz}) ;$ FTIR (film, cm $\left.{ }^{-1}\right) ; 3058(\mathrm{~m}), 2386$ (s), 1598 (m), 1501 (s), 1437 (s), 1108 (m), 1060 (s), 1043 (s), 843 (m); HRMS $(F A B+) \mathrm{m} / \mathrm{z}$ : calcd. $\left(\mathrm{M}^{+}-\mathrm{H}\right) 356.1488$, found 356.1508

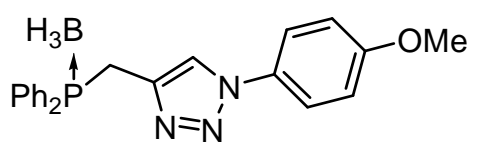

\section{4-((diphenyl(borane)phosphino)methyl)-1-(4-}

methoxyphenyl)-1H-1,2,3-triazole (3c). To a mixture of 2 (32 $\mathrm{mg}, 0.13 \mathrm{mmol}$ ) and 4-methoxyphenylazide $(20 \mathrm{mg}, 0.14 \mathrm{mmol})$ in $t-\mathrm{BuOH}(0.5 \mathrm{~mL})$ and 3 drops of THF a solution of $\mathrm{CuSO}_{4} \cdot 5 \mathrm{H}_{2} \mathrm{O}(3.5 \mathrm{mg}, 0.014 \mathrm{mmol})$ and sodium ascorbate $(5.5 \mathrm{mg}, 0.028$ $\mathrm{mmol}$ ) in $0.5 \mathrm{~mL}$ water was added. The acquired brown suspension is stirred at room temperature for 44 hours. After addition of $12 \mathrm{~mL}$ water the aqueous phase was extracted with EtOAc $(3 \times 7 \mathrm{~mL})$ and the combined organic layers were washed with water $(10 \mathrm{~mL})$ and brine $(10 \mathrm{~mL})$. The organic phase was dried with anhydrous $\mathrm{Na}_{2} \mathrm{SO}_{4}$. Evaporation of solvents afforded product $3 \mathrm{c} \mathrm{(48} \mathrm{mg,93 \% )} \mathrm{as} \mathrm{an} \mathrm{orange}$ solid containing small quantities of starting materials. Recrystallisation from EtOAc gave the product $3 \mathrm{c}(38 \mathrm{mg}, 76 \%)$ as orange crystals: $\mathrm{mp} 141-144{ }^{\circ} \mathrm{C}$. ${ }^{1} \mathrm{H}$ NMR (400 $\mathrm{MHz}) ; \delta(\mathrm{ppm})=7.83(\mathrm{~d}, J=1.9 \mathrm{~Hz}, 1 \mathrm{H}$, triazole-H), 7.76-7.71 $(\mathrm{m}, 4 \mathrm{H}), 7.55-7.49(\mathrm{~m}$, $2 \mathrm{H}), 7.48-7.42(\mathrm{~m}, 6 \mathrm{H}), 6.99-6.96(\mathrm{~m}, 2 \mathrm{H}), 3.87\left(\mathrm{~d}, \mathrm{~J}_{\mathrm{HP}}=11.1 \mathrm{~Hz}, 2 \mathrm{H}, \mathrm{CH}_{2}\right), 3.87(\mathrm{~s}$, $3 \mathrm{H}, \mathrm{OMe}), 1.6-0.6\left(\mathrm{br}, 3 \mathrm{H}, \mathrm{BH}_{3}\right) ;{ }^{13} \mathrm{C}$ NMR $(126 \mathrm{MHz}) ; \delta(\mathrm{ppm})=159.9,139.5(\mathrm{~d}, J=$ $2.1 \mathrm{~Hz}), 132.6$ (d, $J=8.9 \mathrm{~Hz}), 131.7$ (d, $J=2.5 \mathrm{~Hz}), 130.5,129.1$ (d, $J=10.1 \mathrm{~Hz}$ ), $128.4(\mathrm{~d}, J=55.3 \mathrm{~Hz}$ ), 122.2, 121.7 (d, $J=2.5 \mathrm{~Hz}$ ), 114.8, 55.7, 24.8 (d, $J=36.3$ $\mathrm{Hz}) ;{ }^{31} \mathrm{P}$ NMR (162 MHz); $\delta(\mathrm{ppm})=16.3(\mathrm{~d}, J=61.6 \mathrm{~Hz}) ; \mathrm{FTIR}\left(\mathrm{film}, \mathrm{cm}^{-1}\right) ; 3056(\mathrm{w})$, 2385 (s), 1517 (s), 1437 (m), 1255 (s), 1037 (m), 836 (m). 


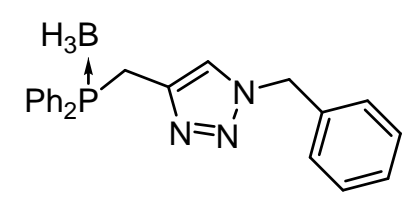

\section{1-benzyl-4-((diphenyl(borane)phosphino)methyl)-1 $H$ -}

1,2,3-triazole (3d). To a mixture of $2(0.24 \mathrm{~g}, 1.0 \mathrm{mmol})$ and sodium ascorbate (40 $\mathrm{mg}, 0.20 \mathrm{mmol})$ in $t-\mathrm{BuOH} / \mathrm{H}_{2} \mathrm{O}(1: 1,4 \mathrm{~mL}), \mathrm{CuSO}_{4} \bullet 5 \mathrm{H}_{2} \mathrm{O}(12 \mathrm{mg}, 0.050 \mathrm{mmol})$ and benzylazide $(0.13 \mathrm{~mL}, 1.0 \mathrm{mmol})$ were added. This white suspension was stirred at room temperature for 7 hours. After addition of $2 \mathrm{~mL}$ water the solvent was removed and the remaining solid was dissolved in EtOAc. This organic layer was washed with water $(5 \mathrm{~mL})$ and brine $(5 \mathrm{~mL})$ and dried with anhydrous $\mathrm{Na}_{2} \mathrm{SO}_{4}$. Product $3 \mathbf{d}$ (340 mg, 92\%) was obtained after evaporation of the solvent. Recrystallisation from EtOAc gave the product (257 mg, 69\%) as yellow crystals: $\mathrm{mp} 108-112{ }^{\circ} \mathrm{C}$. ${ }^{1} \mathrm{H}$ NMR $(400 \mathrm{MHz}) ; \delta(\mathrm{ppm})=7.69-7.64(\mathrm{~m}, 4 \mathrm{H}), 7.46-7.36(\mathrm{~m}, 7 \mathrm{H}), 7.34-7.32(\mathrm{~m}, 3 \mathrm{H}), 7.12-$ $7.10(\mathrm{~m}, 2 \mathrm{H}), 5.41(\mathrm{~s}, 2 \mathrm{H}), 3.76\left(\mathrm{~d}, \mathrm{~J}_{\mathrm{HP}}=11.0 \mathrm{~Hz}, 2 \mathrm{H}, \mathrm{P}-\mathrm{CH}_{2}\right), 1.5-0.5\left(\mathrm{br}, 3 \mathrm{H}, \mathrm{BH}_{3}\right)$; ${ }^{13} \mathrm{C} \mathrm{NMR}(101 \mathrm{MHz}) ; \delta(\mathrm{ppm})=139.5,134.7,132.6(\mathrm{~d}, J=9.0 \mathrm{~Hz}), 131.6(\mathrm{~d}, J=2.3$ $\mathrm{Hz}), 129.2,128.9$ (d, $J=10.2 \mathrm{~Hz}), 128.7,128.3$ (d, $J=55.3 \mathrm{~Hz}), 127.9,123.4,54.2$, $24.8(\mathrm{~d}, J=36.2 \mathrm{~Hz}) ;{ }^{31} \mathrm{P}$ NMR $(162 \mathrm{MHz}) ; \delta(\mathrm{ppm})=16.4(\mathrm{br} \mathrm{d}, J=61.6 \mathrm{~Hz}) ;$ FTIR (film, cm ${ }^{-1}$ ); 3058 (m), 2378 (s), 1545 (w), 1457 (m), 1435 (s), 1340 (m), 1108 (m), $1063(\mathrm{~s}), 1048(\mathrm{~s}), 910(\mathrm{~m}), 845(\mathrm{~m})$.

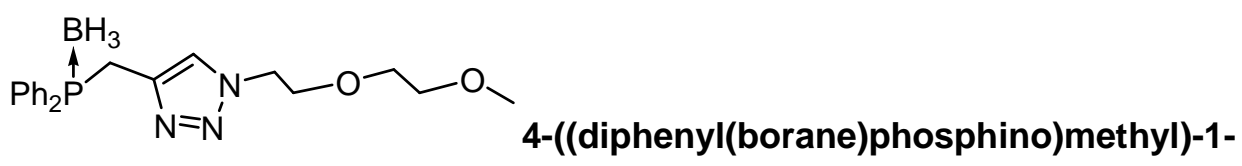

(methoxy ethoxy)ethane-1H-1,2,3-triazole (3e). Phosphine 2 (60 mg, 0.25 mmol, 1 equiv) was mixed with 1-azide-2(2-methoxy ethoxy)ethane (36.6 mg, $0.25 \mathrm{mmol}, 1$ equiv) in $3 \mathrm{~mL}$ of THF. $\mathrm{CuSO}_{4}(10 \% \mathrm{~mol})$ and sodium ascorbate $(20 \% \mathrm{~mol})$ dissolved in $1 \mathrm{~mL}$ of water were added to the solution. The reaction mixture was stirred for $12 \mathrm{~h}$ at room temperature. Subsequently, the solvent was evaporated and a mixture of $\mathrm{CH}_{2} \mathrm{Cl}_{2} / \mathrm{H}_{2} \mathrm{O}$ (10 mL, 1:1) was added. The organic phase was filtered through a pipette packed with $\mathrm{MgSO}_{4}$. Evaporation of solvent afforded product $3 \mathrm{e}$ in $90 \%$ yield $(87 \mathrm{mg})$ as a transparent oil. ${ }^{1} \mathrm{H}$ NMR $(300 \mathrm{MHz}) ; \delta(\mathrm{ppm})=7.74-7.60\left(\mathrm{~m}, 4 \mathrm{H}, \mathrm{C}_{6} \mathrm{H}_{5}\right)$, 7.50-7.34 (m, 7H, $\mathrm{C}_{6} \mathrm{H}_{5}+$ triazole- $\left.\mathrm{H}\right), 4.41\left(\mathrm{t}, 2 \mathrm{H}, \mathrm{N}-\mathrm{CH}_{2}\right), 3.77\left(\mathrm{~d}, 2 \mathrm{H}, \mathrm{P}-\mathrm{CH}_{2}\right), 3.73$ (t, $2 \mathrm{H}, \mathrm{N}-\mathrm{CH}_{2} \mathrm{CH}_{2}$ ), $3.46\left(\mathrm{AB}, 4 \mathrm{H}, \mathrm{OCH}_{2} \mathrm{CH}_{2} \mathrm{O}\right), 3.33$ (t, $\left.3 \mathrm{H}, \mathrm{CH}_{3}\right) \cdot{ }^{13} \mathrm{C} \mathrm{NMR}(\mathrm{MHz}) ; \delta$ $(\mathrm{ppm})=138.8\left(\mathrm{C}_{\text {ipso }}\right.$ triazole $), 132.6,131.7,129.0\left(\mathrm{~d}, \mathrm{C}_{6} \mathrm{H}_{5}\right), 128.3\left(\mathrm{C}_{\mathrm{ipso}} \mathrm{C}_{6} \mathrm{H}_{5}\right), 124.6$ (triazole-C), 71.9, 70.8, $69.7\left(\mathrm{CH}_{2} \mathrm{O}\left(\mathrm{CH}_{2}\right)_{2} \mathrm{O}\right), 59.3\left(\mathrm{CH}_{3}\right), 50.5\left(\mathrm{~N}-\mathrm{CH}_{2}\right), 24.7(\mathrm{~d}, \mathrm{P}-$ $\left.\mathrm{CH}_{2}\right) .{ }^{31} \mathrm{P}$ NMR $(121 \mathrm{MHz}) ; \delta(\mathrm{ppm})=16.6(\mathrm{br})$. 
<smiles>FC(F)(F)c1ccc(-n2cc(CPc3ccccc3)nn2)cc1</smiles>

\section{4-((diphenylphosphino)methyl)-1-(4-}

(trifluoromethyl)phenyl)-1 $\mathbf{H - 1 , 2 , 3 - t r i a z o l e ~ ( 4 a ) . ~ U n d e r ~ a r g o n ~ a t m o s p h e r e ~ a ~ s o l u t i o n ~}$ of $3 \mathrm{a}(0.10 \mathrm{~g}, 0.24 \mathrm{mmol})$ and DABCO $(35 \mathrm{mg}, 0.31 \mathrm{mmol})$ in toluene $(4 \mathrm{~mL}$, filtrated over alumina) was stirred at $70{ }^{\circ} \mathrm{C}$. After $4 \mathrm{~h}$ the solution was cooled to ambient temperature and purified by filtration over a short silica column (EtOAc) to remove DABCO components. Product $4 \mathrm{a}$ was obtained as a yellow/white powder (93 $\mathrm{mg}$, $96 \%)$ after evaporation of solvents. ${ }^{1} \mathrm{H}$ NMR $(400 \mathrm{MHz}) ; \delta(\mathrm{ppm})=7.51$ (s, 4H), 7.49$7.46(\mathrm{~m}, 4 \mathrm{H}), 7.43(\mathrm{~d}, J=0.7 \mathrm{~Hz}, 1 \mathrm{H}$, triazole-H), 7.37-7.35 (m, 6H), $3.62(\mathrm{~s}, 2 \mathrm{H}$, $\left.\mathrm{CH}_{2}\right) ;{ }^{13} \mathrm{C}$ NMR $(101 \mathrm{MHz}) ; \delta(\mathrm{ppm})=145.2(\mathrm{~d}, J=10.4 \mathrm{~Hz}), 139.4,137.4((\mathrm{~d}, J=$ $13.9 \mathrm{~Hz}), 132.8$ (d, $J=18.9 \mathrm{~Hz}), 130.5(\mathrm{q}, J=33.0 \mathrm{~Hz}), 129.1,128.6(\mathrm{~d}, J=6.7 \mathrm{~Hz})$, 127.0 (q, $J=3.7 \mathrm{~Hz}$ ), 123.6 (q, $J=272.3 \mathrm{~Hz}), 120.2,119.4$ (d, $J=6.8 \mathrm{~Hz}), 25.3$ (d, J $=15.6 \mathrm{~Hz}) ;{ }^{31} \mathrm{P}$ NMR $(162 \mathrm{MHz}) ; \delta(\mathrm{ppm})=-14.1$; FTIR $\left(\right.$ film, $\left.\mathrm{cm}^{-1}\right) ; 1617(\mathrm{w}), 1326$ (s), $1169(\mathrm{~m}), 1126$ (s), 1069 (m), 1041 (m), $844(\mathrm{~m})$; HRMS (FAB+) m/z: calcd. $\left(\mathrm{MH}^{+}\right)$412.1190, found 412.1195.<smiles>PCc1cn(-c2ccccc2)nn1</smiles>

\section{4-((diphenylphosphino)methyl)-1-phenyl-1H-1,2,3-triazole}

(4b). Under argon atmosphere a solution of $3 \mathbf{b}(0.20 \mathrm{~g}, 0.56 \mathrm{mmol})$ and DABCO (75 $\mathrm{mg}, 0.67 \mathrm{mmol})$ in THF $(7 \mathrm{~mL})$ was stirred at $67^{\circ} \mathrm{C}$. After $4 \mathrm{~h}$ the solution was cooled to ambient temperature and evaporated to dryness. The resulting solid was dissolved in EtOAc and purified by filtration over a short silica column (EtOAc) to remove DABCO components. Product $\mathbf{4 b}$ (170 mg, 89\%) was obtained as a white solid after evaporation of solvents. ${ }^{1} \mathrm{H}$ NMR $(400 \mathrm{MHz}) ; \delta(\mathrm{ppm})=7.61-7.59(\mathrm{~m}, 2 \mathrm{H}, \mathrm{m}$-Phtriazole), 7.52-7.35 (m, 14H), $3.61\left(\mathrm{~s}, 2 \mathrm{H}, \mathrm{CH}_{2}\right) ;{ }^{13} \mathrm{C} \mathrm{NMR}(101 \mathrm{MHz}) ; \delta(\mathrm{ppm})=144.6$ (d, $J=10.4 \mathrm{~Hz}$ ), 137.7 (d, $J=14.3 \mathrm{~Hz}), 137.1,132.8$ (d, $J=18.8 \mathrm{~Hz}), 129.6,129.0$, $128.6(\mathrm{~d}, J=6.6 \mathrm{~Hz}), 128.5,120.3,119.6(\mathrm{~d}, J=6.7 \mathrm{~Hz}), 25.3(\mathrm{~d}, J=15.4 \mathrm{~Hz}) ;{ }^{31} \mathrm{P}$ $\operatorname{NMR}(162 \mathrm{MHz}) ; \delta(\mathrm{ppm})=-14.3 ; \mathrm{FTIR}\left(\mathrm{film}, \mathrm{cm}^{-1}\right) ; 1598(\mathrm{~m}), 1500$ (s), 1433 (s), $1343(\mathrm{~m}), 1234(\mathrm{~m}), 1042(\mathrm{~s})$.<smiles>COc1ccc(-n2cc(CPc3ccccc3)nn2)cc1</smiles>

\section{4-((diphenylphosphino)methyl)-1-(4-}

methoxyphenyl)-1H-1,2,3-triazole (4c). Under argon atmosphere a solution of 3c (70 mg, $0.18 \mathrm{mmol})$ and DABCO (28 mg, $0.25 \mathrm{mmol})$ in toluene $(4 \mathrm{~mL}$, filtrated over 
alumina) was stirred at $70^{\circ} \mathrm{C}$. After $4 \mathrm{~h}$ the solution was cooled to ambient temperature and purified by filtration over a short silica column (EtOAc) to remove DABCO components. Evaporation of solvents gave product $\mathbf{4 c}$ as a yellow/white powder (59 mg, 87\%). ${ }^{1} \mathrm{H}$ NMR $(400 \mathrm{MHz}) ; \delta(\mathrm{ppm})=7.50-7.46(\mathrm{~m}, 6 \mathrm{H}), 7.37-7.34$ $(\mathrm{m}, 6 \mathrm{H}), 7.31(\mathrm{~m}, 1 \mathrm{H}), 6.97(\mathrm{~m}, 1 \mathrm{H}), 6.96(\mathrm{~m}, 1 \mathrm{H}), 3.84(\mathrm{~s}, 3 \mathrm{H}, \mathrm{OMe}), 3.60(\mathrm{~s}, 2 \mathrm{H}$, $\left.\mathrm{CH}_{2}\right) ;{ }^{13} \mathrm{C}$ NMR $(101 \mathrm{MHz}) ; \delta(\mathrm{ppm})=159.6,144.3(\mathrm{~d}, J=10.4 \mathrm{~Hz}), 137.7(\mathrm{~d}, J=$ $14.1 \mathrm{~Hz}), 132.8$ (d, $J=18.9 \mathrm{~Hz}), 130.6,129.0,128.6$ (d, $J=6.6 \mathrm{~Hz}), 122.0,119.8$ (d, $J=6.7 \mathrm{~Hz}), 114.7,55.6,25.4(\mathrm{~d}, J=15.3 \mathrm{~Hz}) ;{ }^{31} \mathrm{P}$ NMR $(162 \mathrm{MHz}) ; \delta(\mathrm{ppm})=-14.3$; FTIR (film, cm ${ }^{-1}$ ); 1518 (s), 1434 (w), 1256 (m), 1042 (m), 832 (m); HRMS (FAB+) $\mathrm{m} / \mathrm{z}$ : calcd. $\left(\mathrm{MH}^{+}\right)$374.1422, found 374.1418 .

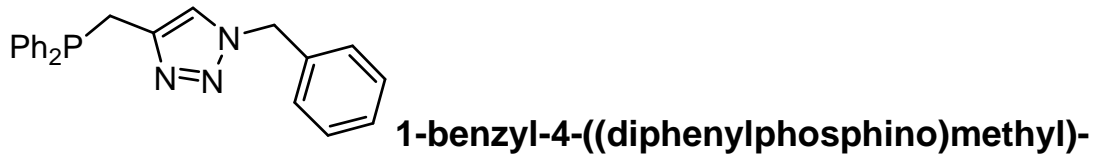

$1 H-1,2,3-$

triazole (4d). Under argon atmosphere a solution of $\mathbf{3 d}(50 \mathrm{mg}, 0.13 \mathrm{mmol})$ and DABCO (23 mg, $0.20 \mathrm{mmol}$ ) in toluene ( $2 \mathrm{~mL}$, filtrated over alumina) was stirred at $70{ }^{\circ} \mathrm{C}$. After $3 \mathrm{~h}$ the solution was cooled to ambient temperature and purified by filtration over a short silica column (EtOAc) to remove DABCO components. The white powder obtained after evaporation of solvents was a mixture of product $\mathbf{4 d}$ (39 $\mathrm{mg}, 83 \%$ ) and its phosphineoxide (4 mg, 9\%, determined by ${ }^{31} \mathrm{P}$ NMR). ${ }^{1} \mathrm{H}$ NMR (400 $\mathrm{MHz}) ; \delta(\mathrm{ppm})=7.42-7.27(\mathrm{~m}, 13 \mathrm{H}), 7.14-7.11(\mathrm{~m}, 2 \mathrm{H}), 6.86(\mathrm{~s}, 1 \mathrm{H}), 5.39(\mathrm{~s}, 2 \mathrm{H})$, $5.39(\mathrm{~s}, 2 \mathrm{H}), 3.50(\mathrm{~s}, 2 \mathrm{H}) ;{ }^{13} \mathrm{C}$ NMR $(101 \mathrm{MHz}) ; \delta(\mathrm{ppm})=144.3(\mathrm{~d}, J=9.9 \mathrm{~Hz}), 137.8$ (d, $J=14.1 \mathrm{~Hz}), 134.9,132.9(\mathrm{~d}, J=18.6 \mathrm{~Hz}), 129.0(\mathrm{~d}, J=14.4 \mathrm{~Hz}), 128.68$, 128.63, 128.56, 128.0, 121.7 (d, $J=6.4 \mathrm{~Hz}$ ), 54.1, 25.6 (d, $J=15.1 \mathrm{~Hz}) ;{ }^{31} \mathrm{P}$ NMR $(162 \mathrm{MHz}) ; \delta(\mathrm{ppm})=-14.3$; HRMS $(\mathrm{FAB}+) \mathrm{m} / \mathrm{z}$ : calcd. $\left(\mathrm{MH}^{+}\right)$358.1473, found 358.1473 .

\footnotetext{
$\mathrm{H}_{3} \mathrm{~B}$<smiles>CP(C[N])c1ccccc1</smiles>
}

Azidomethyl(diphenyl)phosphine borane complex (6). To a solution of hydroxymethyl(diphenyl)phosphine borane complex (1.02 g, $4.42 \mathrm{mmol}$ ) and 2,6lutidine $(0.88 \mathrm{~mL}, 7.52 \mathrm{mmol})$ in chloroform (22 $\mathrm{mL}$, filtrated over alumina) at $-60{ }^{\circ} \mathrm{C}$ $\mathrm{Tf}_{2} \mathrm{O}$ (1.26 mL, $7.52 \mathrm{mmol}$ ) was added under nitrogen atmosphere. The solution was stirred for 3 hours and tetramethylguanidinium azide (TMGA, $3.0 \mathrm{~g}, 19 \mathrm{mmol}$ ) was added. The mixture was stirred overnight allowing to warm to room temperature. 
After $24 \mathrm{~h}$ saturated aqueous ammonium chloride $(100 \mathrm{~mL})$ was added and the mixture was extracted 3 times with $\mathrm{CH}_{2} \mathrm{Cl}_{2}(50 \mathrm{~mL})$. The combined organic layers were washed with water $(2 \times 50 \mathrm{~mL})$ and brine $(50 \mathrm{~mL})$. The water layer $(100 \mathrm{~mL})$ was extracted with EtOAc $(3 \times 70 \mathrm{~mL})$ and the combined organic layers were washed with water $(100 \mathrm{~mL})$ and brine $(100 \mathrm{~mL})$. The organic phase was dried with anhydrous $\mathrm{Na}_{2} \mathrm{SO}_{4}$ and concentrated under reduced pressure. The residu was purified by flash chromatography on silica gel (PE/EtOAc 9:1) to afford 6 as a pale yellow oil $(0.84 \mathrm{~g}, 74 \%)$. ${ }^{1} \mathrm{H}$ NMR $(400 \mathrm{MHz}) ; \delta(\mathrm{ppm})=7.78-7.72(\mathrm{~m}, 4 \mathrm{H}, \mathrm{Ph}), 7.59-$ $7.54(\mathrm{~m}, 2 \mathrm{H}, p-\mathrm{Ph}), 7.52-7.47(\mathrm{~m}, 4 \mathrm{H}, o-\mathrm{Ph}), 4.04\left(\mathrm{~d}, \mathrm{~J}_{\mathrm{HP}}=3.4 \mathrm{~Hz}, 2 \mathrm{H}, \mathrm{CH}_{2}\right), 1.5-0.5$ (br, 3H, BH $) ;{ }^{13} \mathrm{C}$ NMR (126 MHz); $\delta(\mathrm{ppm})=132.9(\mathrm{~d}, J=9.3 \mathrm{~Hz}), 132.2(\mathrm{~d}, J=2.5$ $\mathrm{Hz}), 129.2(\mathrm{~d}, J=10.1 \mathrm{~Hz}), 126.5(\mathrm{~d}, J=55.3 \mathrm{~Hz}), 48.6(\mathrm{~d}, J=37.1 \mathrm{~Hz}) ;{ }^{31} \mathrm{P}$ NMR $(162 \mathrm{MHz}) ; \delta(\mathrm{ppm})=19.8(\mathrm{~d}, J=61.6 \mathrm{~Hz}) ; \mathrm{FTIR}$ (film, cm$\left.{ }^{-1}\right) ; 3058(\mathrm{w}), 2389$ (s), 2092 (s), 1437 (s), 1250 (m), 1107 (m), 1061 (s), 858 (w).<smiles>FC(F)(F)c1ccc(-c2cn(CP)nn2)cc1</smiles>

\section{1-((diphenyl(borane)phosphino)methyl)-4-(4-}

trifluoromethylphenyl)-1H-1,2,3-triazole (7a). Azidomethyl(diphenyl)phosphine borane complex $6(0.10 \mathrm{~g}, 0.39 \mathrm{mmol})$, 4-trifluoromethylphenylacetylene $(66 \mu \mathrm{L}, 0.39$ mmol), sodium ascorbate (31 mg, $0.16 \mathrm{mmol})$, and $\mathrm{CuSO}_{4} \bullet 5 \mathrm{H}_{2} \mathrm{O}(10 \mathrm{mg}, 0.039$ $\mathrm{mmol})$ were suspended in a mixture of water and $t-\mathrm{BuOH}(2 \mathrm{~mL}, 1: 1)$. This mixture was stirred at room temperature for 3.5 hours. The white suspension was extracted once with EtOAc $(10 \mathrm{~mL})$ and the organic layer was washed two times with $\mathrm{H}_{2} \mathrm{O}(9$ $\mathrm{mL}$ ). The solution was dried with anhydrous $\mathrm{Na}_{2} \mathrm{SO}_{4}$ and concentrated under reduced pressure. The residu was purified by flash chromatography on silica gel (PE/EtOAc 2:1) resulting after evaporation of solvents in compound 7a as a white solid (118 mg, 71\%): mp 124-127 ${ }^{\circ}$. ${ }^{1} \mathrm{H}$ NMR (400 MHz); $\delta(\mathrm{ppm})=7.90(\mathrm{~s}, 1 \mathrm{H}$, triazole-H), $7.85(\mathrm{~d}, J=8.1 \mathrm{~Hz}, 2 \mathrm{H}), 7.75-7.70(\mathrm{~m}, 4 \mathrm{H}), 7.65(\mathrm{~d}, J=8.3 \mathrm{~Hz}, 2 \mathrm{H})$, 7.59-7.54 (m, 2H), 7.51-7.47 (m, 4H), $5.32\left(\mathrm{~d}, \mathrm{~J}_{\mathrm{HP}}=4.1 \mathrm{~Hz}, 2 \mathrm{H}, \mathrm{CH}_{2}\right), 1.6-0.6(\mathrm{br}, 3 \mathrm{H}$, $\left.\mathrm{BH}_{3}\right) ;{ }^{13} \mathrm{C}$ NMR $(101 \mathrm{MHz}) ; \delta(\mathrm{ppm})=146.7,133.7,132.9(\mathrm{~d}, J=9.8 \mathrm{~Hz}), 132.6(\mathrm{~d}, J$ $=2.5 \mathrm{~Hz}$ ), 130.3 (q, $J=32.4 \mathrm{~Hz}), 129.4$ (d, $J=10.4 \mathrm{~Hz}), 126.01,125.96$ (q, $J=3.9$ $\mathrm{Hz}), 125.1$ (d, $J=55.3 \mathrm{~Hz}$ ), 124.2 (q, $J=272.0 \mathrm{~Hz}$ ), 122.0, 48.1 (d, $J=34.9 \mathrm{~Hz}$ ); ${ }^{31} \mathrm{P}$ NMR (162 MHz); $\delta(p p m)=17.9$ (br); FTIR (film, cm ${ }^{-1}$ ); 3059 (w), 2393 (s), 1622 (w), 1438 (w), 1326 (s), $1167(\mathrm{~m}), 1124$ (s), 1063 (s), 850 (w). 


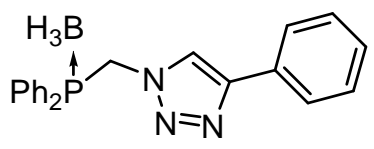

\section{1-((diphenyl(borane)phosphino)methyl)-4-phenyl-1 H-}

1,2,3-triazole (7b). To a solution of azidomethyl(diphenyl)phosphine borane complex $6(0.15 \mathrm{~g}, 0.59 \mathrm{mmol})$ in $t$ - $\mathrm{BuOH}(4 \mathrm{~mL})$ phenylacetylene $(88 \mu \mathrm{L}, 0.80 \mathrm{mmol})$ was added, together with a solution of sodium ascorbate $(47 \mathrm{mg}, 0.24 \mathrm{mmol}$ ) in $1 \mathrm{~mL}$ water and a solution of $\mathrm{CuSO}_{4} \bullet 5 \mathrm{H}_{2} \mathrm{O}(15 \mathrm{mg}, 0.059 \mathrm{mmol})$ in $1 \mathrm{~mL}$ water. This mixture was stirred at room temperature for 4 hours under nitrogen atmosphere. The white suspension was extracted once with EtOAc $(15 \mathrm{~mL})$ and the organic layer was evaporated to dryness. The residu was purified by flash chromatography on silica gel (first $\mathrm{CH}_{2} \mathrm{Cl}_{2}$ followed by pure EtOAc) resulting after evaporation of solvents in compound $7 \mathrm{~b}$ as a yellow foam (185 mg, 88\%): mp 107-111 ${ }^{\circ} \mathrm{C} .{ }^{1} \mathrm{H}$ NMR $(400 \mathrm{MHz})$; $\delta(\mathrm{ppm})=7.77(\mathrm{~s}, 1 \mathrm{H}$, triazole- $\mathrm{H}), 7.75-7.68(\mathrm{~m}, 6 \mathrm{H}, \mathrm{Ph}), 7.58-7.29(\mathrm{~m}, 9 \mathrm{H}, \mathrm{Ph}), 5.30$ $\left(\mathrm{d}, J_{\mathrm{HP}}=3.9 \mathrm{~Hz}, 2 \mathrm{H}, \mathrm{CH}_{2}\right), 1.6-0.6\left(\mathrm{br}, 3 \mathrm{H}, \mathrm{BH}_{3}\right) ;{ }^{13} \mathrm{C} \mathrm{NMR}(101 \mathrm{MHz}) ; \delta(\mathrm{ppm})=$ 148.2, 133.0 (d, $J=9.6 \mathrm{~Hz}), 132.6$ (d, $J=2.3 \mathrm{~Hz}$ ), 130.3, 129.5 (d, $J=10.3 \mathrm{~Hz}$ ), 129.0, 128.5, 126.0, 125.3 (d, $J=55.4 \mathrm{~Hz}$ ), 121.2, 48.2 (d, $J=34.6 \mathrm{~Hz}$ ); ${ }^{31} \mathrm{P}$ NMR $(162 \mathrm{MHz}) ; \delta(\mathrm{ppm})=18.0(\mathrm{~d}, J=51.8 \mathrm{~Hz}) ; \mathrm{FTIR}\left(\mathrm{film}, \mathrm{cm}^{-1}\right) ; 3059(\mathrm{w}), 2392(\mathrm{~s})$, $1484(w), 1437$ (s), 1230 (w), $1192(w), 1108(m), 1060(m), 1040(m), 911(w), 854$ $(w)$.

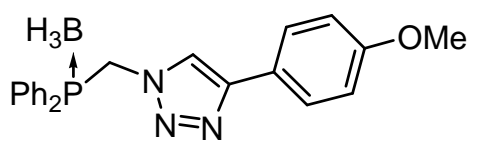

\section{1-((diphenyl(borane)phosphino)methyl)-4-(4-}

methoxyphenyl)-1H-1,2,3-triazole (7c). Azidomethyl(diphenyl)phosphine borane complex 6 (0.10 g, $0.39 \mathrm{mmol})$, 4-methoxyphenylacetylene (54 mg, $0.41 \mathrm{mmol})$, sodium ascorbate $(31 \mathrm{mg}, 0.16 \mathrm{mmol})$, and $\mathrm{CuSO}_{4} \bullet 5 \mathrm{H}_{2} \mathrm{O}(10 \mathrm{mg}, 0.039 \mathrm{mmol})$ were suspended in a mixture of water $(1.5 \mathrm{~mL})$ and $t-\mathrm{BuOH}(3.0 \mathrm{~mL})$. This mixture was stirred at room temperature for 5 hours. The suspension was extracted once with EtOAc $(15 \mathrm{~mL})$ and the organic layer was evaporated to dryness. The residu was purified by flash chromatography on silica gel (gradient EtOAc/PE (1:8 to 1:1)) resulting after evaporation of solvents in compound 7c as a white solid (106 mg, 70\%): mp 132-137 ${ }^{\circ}$. ${ }^{1} \mathrm{H}$ NMR $(400 \mathrm{MHz}) ; \delta(\mathrm{ppm})=7.74-7.63(\mathrm{~m}, 7 \mathrm{H}), 7.57-7.45$ $(\mathrm{m}, 6 \mathrm{H}), 6.94-6.91(\mathrm{~m}, 2 \mathrm{H}), 5.28\left(\mathrm{~d}, J_{\mathrm{HP}}=3.8 \mathrm{~Hz}, 2 \mathrm{H}, \mathrm{CH}_{2}\right), 3.83(\mathrm{~s}, 3 \mathrm{H}, \mathrm{OMe}), 1.6-$ $0.6\left(\mathrm{br}, 3 \mathrm{H}, \mathrm{BH}_{3}\right) ;{ }^{13} \mathrm{C}$ NMR $(101 \mathrm{MHz}) ; \delta(\mathrm{ppm})=159.8,147.9,132.9(\mathrm{~d}, J=9.7 \mathrm{~Hz})$, 132.5 (d, $J=2.5 \mathrm{~Hz}$ ), 129.4 (d, $J=10.3 \mathrm{~Hz}), 127.2,125.3$ (d, $J=55.5 \mathrm{~Hz}$ ), 123.0, 120.3, 114.4, 55.5, 48.1 (d, $J=34.7 \mathrm{~Hz}) ;{ }^{31} \mathrm{P}$ NMR (162 MHz); $\delta(\mathrm{ppm})=18.0(\mathrm{br})$; 
FTIR (film, cm ${ }^{-1}$ ); 3054 (w), 2935 (w), 2835 (w), 2390 (s), 1616 (w), 1562 (w), 1496 (s), $1441(\mathrm{~s}), 1251$ (s), $1065(\mathrm{~m}), 1030(\mathrm{~s}), 840(\mathrm{~m})$.<smiles>FC(F)(F)c1ccc(-c2cn(CPc3ccccc3)nn2)cc1</smiles>

\section{1-((diphenyl(borane)phosphino)methyl)-4-(4-}

(trifluoromethyl)phenyl)-1 $\mathbf{H - 1 , 2 , 3 - t r i a z o l e ~ ( 8 a ) . ~ U n d e r ~ a r g o n ~ a t m o s p h e r e ~ a ~ s o l u t i o n ~}$ of borane protected phosphine 7a (50 mg, $0.12 \mathrm{mmol}$ ) and DABCO (16 mg, 0.14 $\mathrm{mmol})$ in dry toluene $(2 \mathrm{~mL})$ was stirred at $70^{\circ} \mathrm{C}$. A fter $2 \mathrm{~h}$ the solution was cooled to ambient temperature and purified by filtration over a short silica column (toluene as eluent) to remove DABCO components. Evaporation of solvents gave product $8 \mathbf{a}$ as a white solid (42 mg, 86\%). ${ }^{1} \mathrm{H}$ NMR (400 MHz); $\delta(\mathrm{ppm})=7.84(\mathrm{~d}, 2 \mathrm{H}, J=8.1 \mathrm{~Hz})$, $7.65(\mathrm{~d}, 2 \mathrm{H}, J=8.2 \mathrm{~Hz}) 7.61\left(\mathrm{~s}, 1 \mathrm{H}\right.$, triazole-H), 7.48-7.38 $(\mathrm{m}, 10 \mathrm{H}), 5.15\left(\mathrm{~d}, J_{\mathrm{HP}}=\right.$ $\left.5.2 \mathrm{~Hz}, 2 \mathrm{H}, \mathrm{CH}_{2}\right) ;{ }^{13} \mathrm{C} \operatorname{NMR}(101 \mathrm{MHz}) ; \delta(\mathrm{ppm})=146.6,134.4(\mathrm{~d}, J=12.2 \mathrm{~Hz})$, 134.1 (d, $J=1.1 \mathrm{~Hz}$ ), 133.1 (d, $J=19.5 \mathrm{~Hz}), 130.0$ (q, $J=32.5 \mathrm{~Hz}$ ), 130.0, 129.2 (d, $J=6.9 \mathrm{~Hz}$ ), 125.9-126.0 (s+q, 4C), 124.2 (q, $J=271.9 \mathrm{~Hz}), 120.6$ (d, $J=3.7 \mathrm{~Hz}$ ), $50.4(\mathrm{~d}, J=20.9 \mathrm{~Hz}) ;{ }^{31} \mathrm{P}$ NMR (162 MHz); $\delta$ (ppm) = -13.8; FTIR (film, $\mathrm{cm}^{-1}$ ); 1621 (w), 1435 (w), 1326 (s), $1166(\mathrm{~m}), 1122(\mathrm{~m}), 1063$ (m), 849 (w); HRMS (FAB+) m/z: calcd. $\left(\mathrm{MH}^{+}\right)$412.1190, found 412.1195 .<smiles>PCn1cc(-c2ccccc2)nn1</smiles>

\section{1-((diphenyl(borane)phosphino)methyl)-4-phenyl-1 H-}

1,2,3-triazole (8b). Under argon atmosphere a solution of borane protected phosphine $7 \mathrm{~b}$ (30 mg, $0.085 \mathrm{mmol})$ and DABCO (18 mg, $0.16 \mathrm{mmol})$ in toluene (1.5 $\mathrm{mL}$, filtrated over alumina) was stirred at $70{ }^{\circ} \mathrm{C}$. A fter $5 \mathrm{~h}$ the solution was cooled to ambient temperature and purified by filtration over a short silica column (EtOAc) to remove DABCO components. Evaporation of solvents gave product $\mathbf{8 b}$ as a white solid (20 mg, 69\%). Also oxidised material (9 mg, 30\%) was recovered as a second fraction. ${ }^{1} \mathrm{H}$ NMR $(400 \mathrm{MHz}) ; \delta(\mathrm{ppm})=7.75-7.73(\mathrm{~m}, 2 \mathrm{H}), 7.57(\mathrm{~s}, 1 \mathrm{H}$, triazole-H), 7.48-7.38 (m, 12H), 7.33-7.29 (m, 1H), $5.13\left(\mathrm{~d}, \mathrm{~J}_{\mathrm{HP}}=5.1 \mathrm{~Hz}, 2 \mathrm{H}, \mathrm{CH}_{2}\right) ;{ }^{13} \mathrm{C} \mathrm{NMR}$ $(101 \mathrm{MHz}) ; \delta(\mathrm{ppm})=148.0,134.5(\mathrm{~d}, J=12.4 \mathrm{~Hz}), 133.1(\mathrm{~d}, J=19.2 \mathrm{~Hz}), 130.7$, 129.9, 129.1 (d, $J=7.0 \mathrm{~Hz}$ ), 128.9, 128.2, 125.8, 119.8 (d, $J=3.7 \mathrm{~Hz}), 50.4$ (d, $J=$ $20.5 \mathrm{~Hz}) ;{ }^{31} \mathrm{P}$ NMR (162 MHz); $\delta(\mathrm{ppm})=-14.1$; FTIR (film, $\left.\mathrm{cm}^{-1}\right) ; 3053(\mathrm{~m}), 1482(\mathrm{~m})$, $1461(\mathrm{~m}), 1434(\mathrm{~s}), 1224(\mathrm{w}), 1188(\mathrm{~m}), 1096(\mathrm{~m}), 1074(\mathrm{~m}), 1042(\mathrm{~s}), 1026(\mathrm{~m}), 914$ (w). 


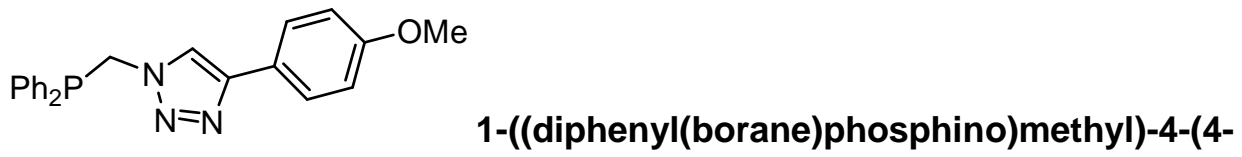

methoxyphenyl)-1H-1,2,3-triazole (8c). Under argon atmosphere a solution of borane protected phosphine $7 \mathrm{c}(40 \mathrm{mg}, 0.11 \mathrm{mmol})$ and DABCO (18 mg, $0.16 \mathrm{mmol})$ in toluene $\left(1.5 \mathrm{~mL}\right.$, filtrated over alumina) was stirred at $70{ }^{\circ} \mathrm{C}$. After $5 \mathrm{~h}$ the solution was cooled to ambient temperature and purified by filtration over a short silica column (EtOAc) to remove DABCO components. Evaporation of solvents gave product $8 \mathrm{c}$ as a slightly brown/white solid (24 mg, 62\%). ${ }^{1} \mathrm{H}$ NMR $(400 \mathrm{MHz}) ; \delta$ $(\mathrm{ppm})=7.68-7.65(\mathrm{~m}, 2 \mathrm{H})$, 7.49-7.37 $(\mathrm{m}, 11 \mathrm{H}), 6.95-6.91(\mathrm{~m}, 2 \mathrm{H}), 5.11\left(\mathrm{~d}, J_{\mathrm{HP}}=5.0\right.$ $\mathrm{Hz}, 2 \mathrm{H}, \mathrm{CH}_{2}$ ), $3.82(\mathrm{~s}, 3 \mathrm{H}, \mathrm{OMe}) ;{ }^{13} \mathrm{C} \mathrm{NMR}(101 \mathrm{MHz}) ; \delta(\mathrm{ppm})=159.8,147.8,134.6$ (d, $J=12.2 \mathrm{~Hz}$ ), 133.1 (d, $J=19.3 \mathrm{~Hz}$ ), 130.0, 129.1 (d, $J=7.0 \mathrm{~Hz}$ ), 127.2, 123.2, $119.1(\mathrm{~d}, J=3.8 \mathrm{~Hz}), 114.4,55.5,50.6$ (d, $J=20.4 \mathrm{~Hz}) ;{ }^{31} \mathrm{P}$ NMR $(162 \mathrm{MHz}) ; \delta$ (ppm) $=-14.2 ; \mathrm{HRMS}(\mathrm{FAB}+) \mathrm{m} / \mathrm{z}$ : calcd. $\left(\mathrm{MH}^{+}\right) 374.1422$, found 374.1418 .

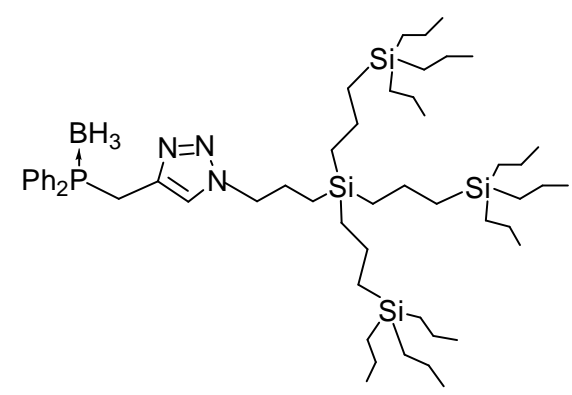

\section{1-((diphenyl(borane)phosphino)methyl)-4-}

dend-1H-1,2,3-triazole (10). Phosphine 2 (67 mg, $0.28 \mathrm{mmol}, 1$ equiv) was mixed with dendrimer 9 (200 mg, $0.28 \mathrm{mmol}, 1$ equiv) in $3 \mathrm{~mL}$ of $\mathrm{THF}^{-\mathrm{CuSO}_{4}}$ (0.1 equiv) and sodium ascorbate ( 0.2 equiv) dissolved in $1 \mathrm{~mL}$ of water were added to the solution. The reaction mixture was stirred for $12 \mathrm{~h}$ at room temperature. Subsequently, the solvent was evaporated and a mixture of $\mathrm{Et}_{2} \mathrm{O} / \mathrm{H}_{2} \mathrm{O}(10 \mathrm{~mL}, 1: 1)$ was added. The organic phase was filtered through a pipette packed with $\mathrm{MgSO}_{4}$. Dendrimer 10 was obtained as a transparent oil in 95\% yield (253 mg). ${ }^{1} \mathrm{H}$ NMR (300 $\mathrm{MHz}) ; \delta(\mathrm{ppm})=7.72-7.65\left(\mathrm{~m}, 4 \mathrm{H}, \mathrm{C}_{6} \mathrm{H}_{5}\right), 7.45-7.38\left(\mathrm{~m}, 7 \mathrm{H}, \mathrm{C}_{6} \mathrm{H}_{5}+\right.$ triazole- $\left.\mathrm{H}\right), 4.17$ (t, $2 \mathrm{H}, \mathrm{N}-\mathrm{CH}_{2}$ ), 3.77 (d, 2H, P- $\left.\mathrm{CH}_{2}\right), 1.67\left(\mathrm{~m}, 2 \mathrm{H}, \mathrm{N}-\mathrm{CH}_{2} \mathrm{CH}_{2}\right), 1.39-1.21(\mathrm{~m}, 24 \mathrm{H}$, $\mathrm{CH}_{2}$-dend), 0.93 (t, 27H, $\mathrm{CH}_{3}$ ), 0.59-0.44 (m, 32H, $\mathrm{CH}_{2}$-dend). ${ }^{13} \mathrm{C} \mathrm{NMR}(75 \mathrm{MHz}) ; \delta$ $(\mathrm{ppm})=138.8\left(\mathrm{C}_{\text {ipso }}\right.$ triazole $), 132.6,131.6,129.0\left(\mathrm{~d}, \mathrm{C}_{6} \mathrm{H}_{5}\right), 128.3\left(\mathrm{C}_{\text {ipso }} \mathrm{C}_{6} \mathrm{H}_{5}\right), 123.2$ (triazole-C), $53.7\left(\mathrm{~N}-\mathrm{CH}_{2}\right), 24.9$ (d, P- $\left.\mathrm{CH}_{2}\right), 25.6,18.9,18.8,18.0,17.7,15.6,10.0$ (dend). ${ }^{31} \mathrm{P}$ NMR $(121 \mathrm{MHz}) ; \delta(\mathrm{ppm})=16.4(\mathrm{br})$. 


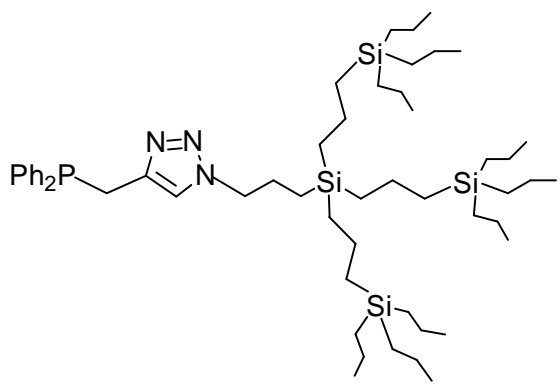

1-((diphenylphosphino)methyl)-4-dend-1H-

1,2,3-triazole (11). A solution of dendrimer 10 (150 mg, $0.16 \mathrm{mmol}, 1$ equiv) and DABCO (21.3 mg, $0.19 \mathrm{mmol}, 1.2$ equiv) in toluene $(10 \mathrm{~mL})$ was stirred at $70{ }^{\circ} \mathrm{C}$ under argon atmosphere. After $3 \mathrm{~h}$ the solvent was removed and the product was purified by filtrating it through a pipette packed with silica using $\mathrm{Et}_{2} \mathrm{O}$ as eluent. Dendrimer 11 was obtained as a transparent oil in $95 \%$ yield $(140.3 \mathrm{mg}) .{ }^{1} \mathrm{H}$ NMR $(300 \mathrm{MHz}) ; \delta(\mathrm{ppm})=7.46-7.40\left(\mathrm{~m}, 4 \mathrm{H}, \mathrm{C}_{6} \mathrm{H}_{5}\right), 7.35-7.30\left(\mathrm{~m}, 6 \mathrm{H}, \mathrm{C}_{6} \mathrm{H}_{5}\right), 6.94(\mathrm{~s}, 1 \mathrm{H}$, triazole-H), 4.15 (t, $\left.2 \mathrm{H}, \mathrm{N}-\mathrm{CH}_{2}\right), 3.51\left(\mathrm{~d}, 2 \mathrm{H}, \mathrm{P}-\mathrm{CH}_{2}\right), 1.73\left(\mathrm{~m}, 2 \mathrm{H}, \mathrm{N}-\mathrm{CH}_{2} \mathrm{CH}_{2}\right), 1.39$ $1.21\left(\mathrm{~m}, 24 \mathrm{H}, \mathrm{CH}_{2}\right.$-dend), $0.95\left(\mathrm{t}, 27 \mathrm{H}, \mathrm{CH}_{3}\right), 0.60-0.40\left(\mathrm{~m}, 32 \mathrm{H}, \mathrm{CH}_{2}\right.$-dend). ${ }^{13} \mathrm{C}$ $\operatorname{NMR}(101 \mathrm{MHz}) ; \delta(\mathrm{ppm})=143.6\left(\mathrm{~d}, J=10.5 \mathrm{~Hz}, \mathrm{C}_{\text {ipso }}\right.$ triazole $), 137.9(\mathrm{~d}, J=14.2$ $\left.\mathrm{Hz}, \mathrm{C}_{\text {ipso }} \mathrm{C}_{6} \mathrm{H}_{5}\right), 132.8$ (d, $\left.J=18.8 \mathrm{~Hz}, \mathrm{C}_{6} \mathrm{H}_{5}\right), 128.8,128.5\left(\mathrm{~d}, J=18.8 \mathrm{~Hz}, \mathrm{C}_{6} \mathrm{H}_{5}\right)$, $121.1\left(\mathrm{~d}, J=6.7 \mathrm{~Hz}\right.$, triazole-C), $53.6\left(\mathrm{~N}-\mathrm{CH}_{2}\right), 29.7,25.4\left(\mathrm{~d}, J=14.1 \mathrm{~Hz}, \mathrm{P}-\mathrm{CH}_{2}\right)$, 18.7, 18.6, 17.8, 17.5, 15.6, 9.8 (dend). ${ }^{31} \mathrm{P}$ NMR (162 MHz); $\delta(p p m)=-14.0$.

Azidomethyl PS-resin (13). To a suspension of chloromethyl-polystyrene resin (Fluka Merrifield polymer crosslinked with 1\% divinylbenzene (DVB); 200-400 mesh; $0.8 \mathrm{mmol} \mathrm{Cl} / \mathrm{g} ; 2.0 \mathrm{~g}, 1.6 \mathrm{mmol}$ ) in DMSO (20 mL) sodium azide (520 mg, 8.0 $\mathrm{mmol})$ was added. The reaction was performed in a scintillation vessel, bubbling nitrogen gas through the resin suspension. The suspension was allowed to react for 3 days at $60{ }^{\circ} \mathrm{C}$. After being cooled to room tempera ture, the suspension was filtrated and the resin was washed alternatingly with $\mathrm{MeOH}(5 \times 12 \mathrm{~mL})$ and $\mathrm{DCM}(5 \times 12 \mathrm{~mL})$ to give azidomethyl polystyrene 13. FTIR $\left(\mathrm{KBr}, \mathrm{cm}^{-1}\right) ; 2094$ (s).

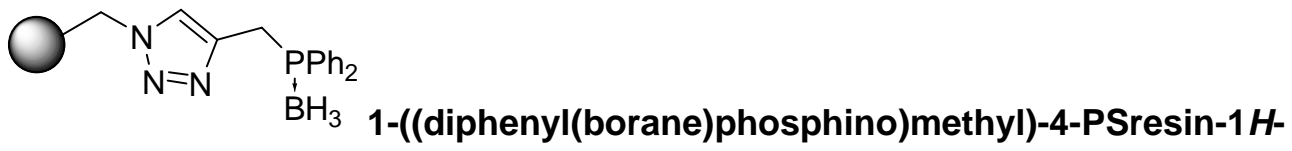

1,2,3-triazole (14). Azidomethyl PS-resin 13 (300 mg, $\left.0.8 \mathrm{mmol} \mathrm{N}_{3} / \mathrm{g}\right)$, phosphine 2 (171 mg, $0.72 \mathrm{mmol}$ ), Cul (5 mg, $0.024 \mathrm{mmol}$ ), TBTA (14 mg, $0.026 \mathrm{mmol}$ ) and DiPEA $(0.13 \mathrm{~mL}, 0.72 \mathrm{mmol})$ were suspended in THF $(3 \mathrm{~mL})$. The reaction was run under a nitrogen atmosphere in Radleys Carousel Reaction Station ${ }^{\mathrm{TM}}$ using a 
modified glass reaction tube. The tube was fitted with a glass frit and luer tip to facilitate work-up on the IST VacMaster-20 Sample Processing Station ${ }^{\mathrm{TM}}$. The reaction was gently stirred with a magnetic stirring bar at $40{ }^{\circ} \mathrm{C}$ for $20 \mathrm{~h}$. After filtration and washing (same as described below) the resin was reloaded with half the amounts used for the first load to obtain complete conversion of the azide (disappearance of azide signal in FTIR). After $40 \mathrm{~h}$ the suspension was filtrated and the resin was washed alternatingly with $\mathrm{MeOH}(3 \times 2 \mathrm{~mL})$, pyridine $(3 \times 2 \mathrm{~mL})$, and DCM $(3 \times 2 \mathrm{~mL})$ followed by two additional washing steps with diethyl ether. The resin was dried under vacuum to give "clicked" resin 14 (max. loading $0.67 \mathrm{mmol} / \mathrm{g}$ ). ${ }^{31} \mathrm{P}$ MAS NMR $(203 \mathrm{MHz}) ; \delta(\mathrm{ppm})=17.9$.

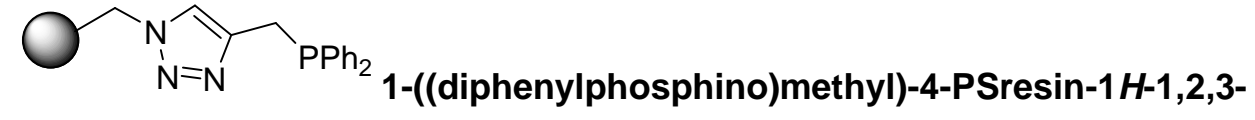

triazole (15). Supported ligand 14 (236 $\mathrm{mg}, 0.67 \mathrm{mmol} / \mathrm{g}$ loading) was mixed with DABCO (88.7 mg, 5 equiv) in $10 \mathrm{~mL}$ of toluene. The mixture was stirred under a $\mathrm{N}_{2}$ flow for $8 \mathrm{~h}$ at $70{ }^{\circ} \mathrm{C}$. Subsequently, the solvent was removed and the resin was washed with toluene $(4 \times 7 \mathrm{~mL})$ and dried under vacuum. ${ }^{31} \mathrm{P}$ MAS NMR $(203 \mathrm{MHz}) ; \delta$ $(\mathrm{ppm})=-13.2$.

\section{Typical experimental procedure for the preparation of palladium complexes:}

Ligand 4b (62 mg, $0.18 \mathrm{mmol}, 1$ equiv) was mixed with [PdallylCl$]_{2}$ (33 mg, 0.09 mmol, 0.5 equiv) in $7 \mathrm{~mL}$ of $\mathrm{CH}_{2} \mathrm{Cl}_{2}$. The resulting solution was stirred at room temperature for $45 \mathrm{~min}$ under argon affording complex $17 \mathrm{~b}$. To the mixture was added $\mathrm{AgBF}_{4}$ (35 mg, $0.18 \mathrm{mmol}, 1$ equiv): formation of a precipitate $(\mathrm{AgCl})$ was observed. After $1 \mathrm{~h}$ stirring at room temperature the crude mixture was filtered through a pipette packed with Celite. Evaporation of the solvent afforded the cationic $\mathrm{Pd}$ complex $\mathbf{1 8 b}$ as a foamy solid in quantitative yield. 
NMR spectra.

$2{ }^{1} \mathrm{H}$ NMR in $\mathrm{CDCl}_{3}$<smiles>[B]P(CC#C)c1ccccc1</smiles>

步
m
-1
0
0
.7
-7
0
$\overrightarrow{1}$
$\overrightarrow{0}$

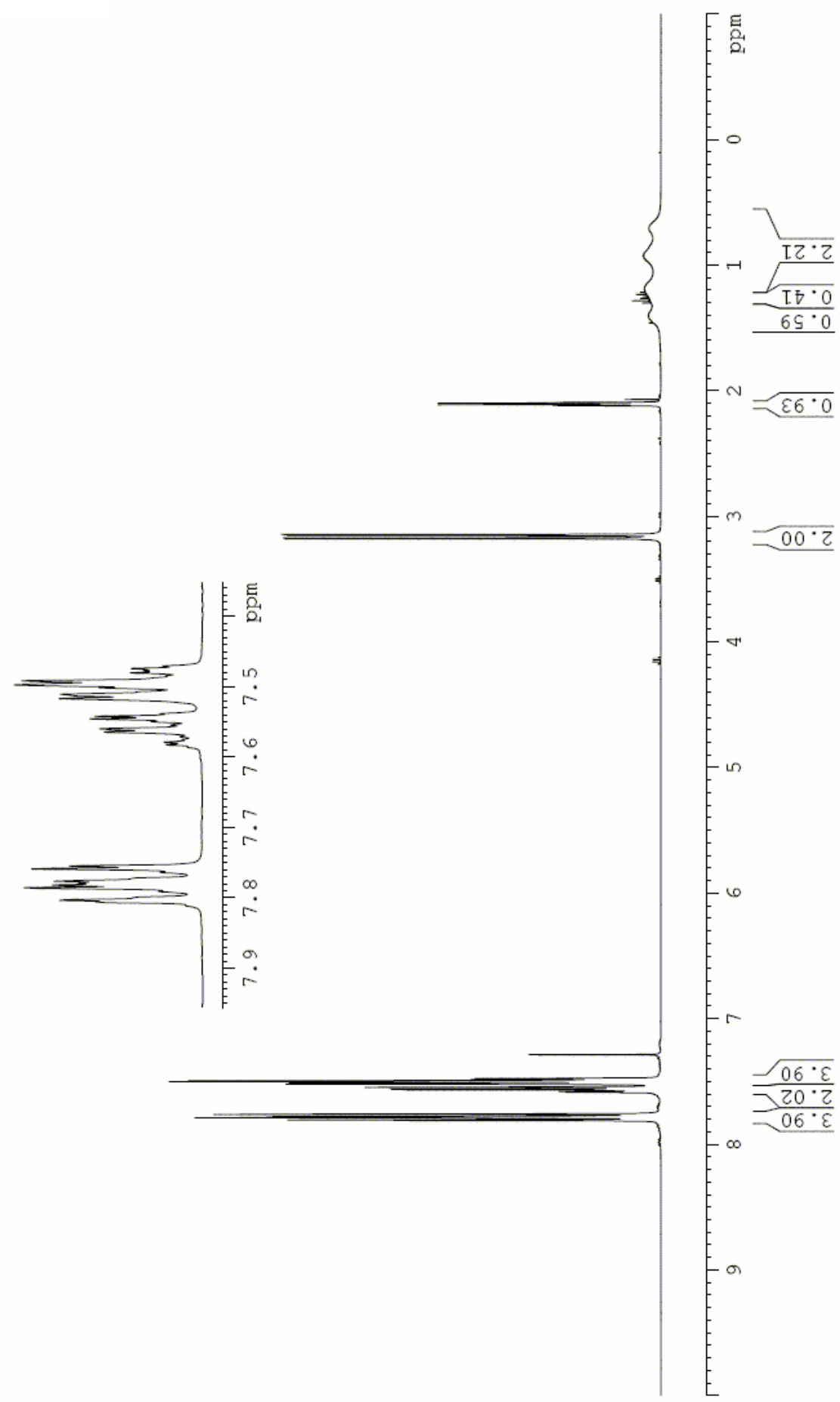




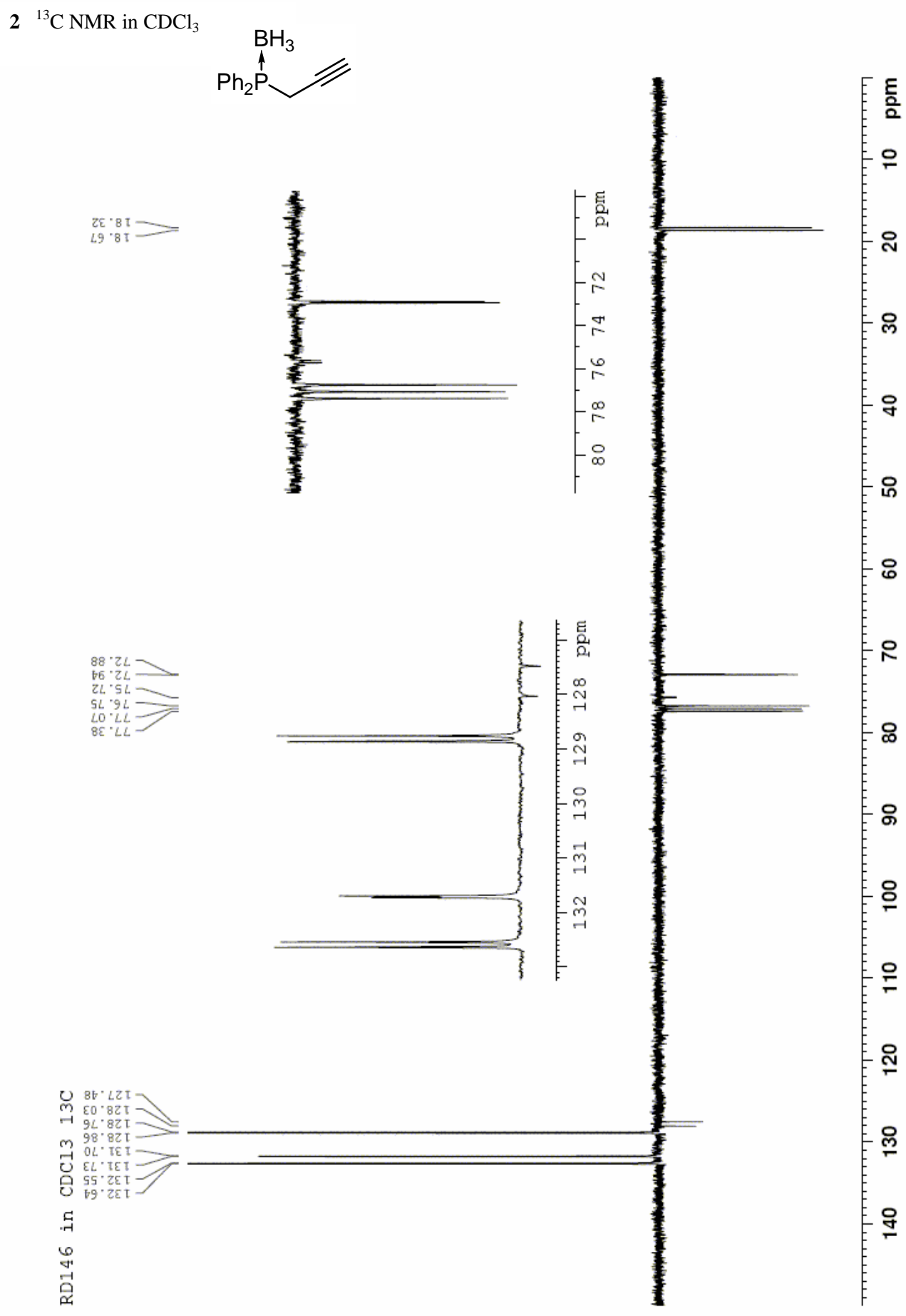


$2{ }^{31} \mathrm{P} \mathrm{NMR}$ in $\mathrm{CDCl}_{3}$
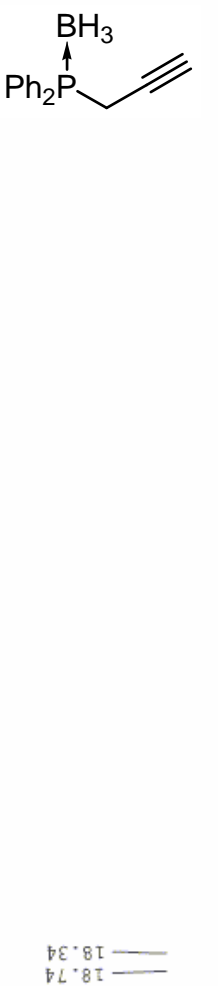

ल

苮

.

品

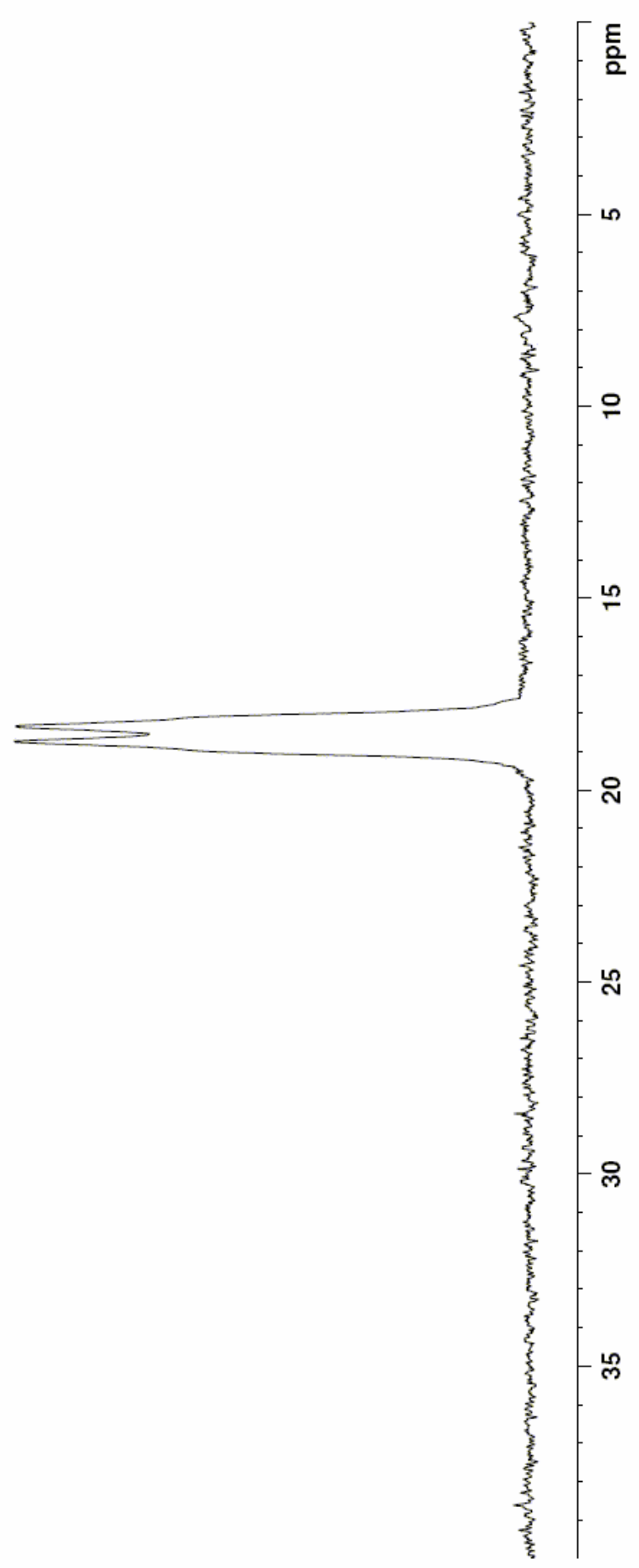




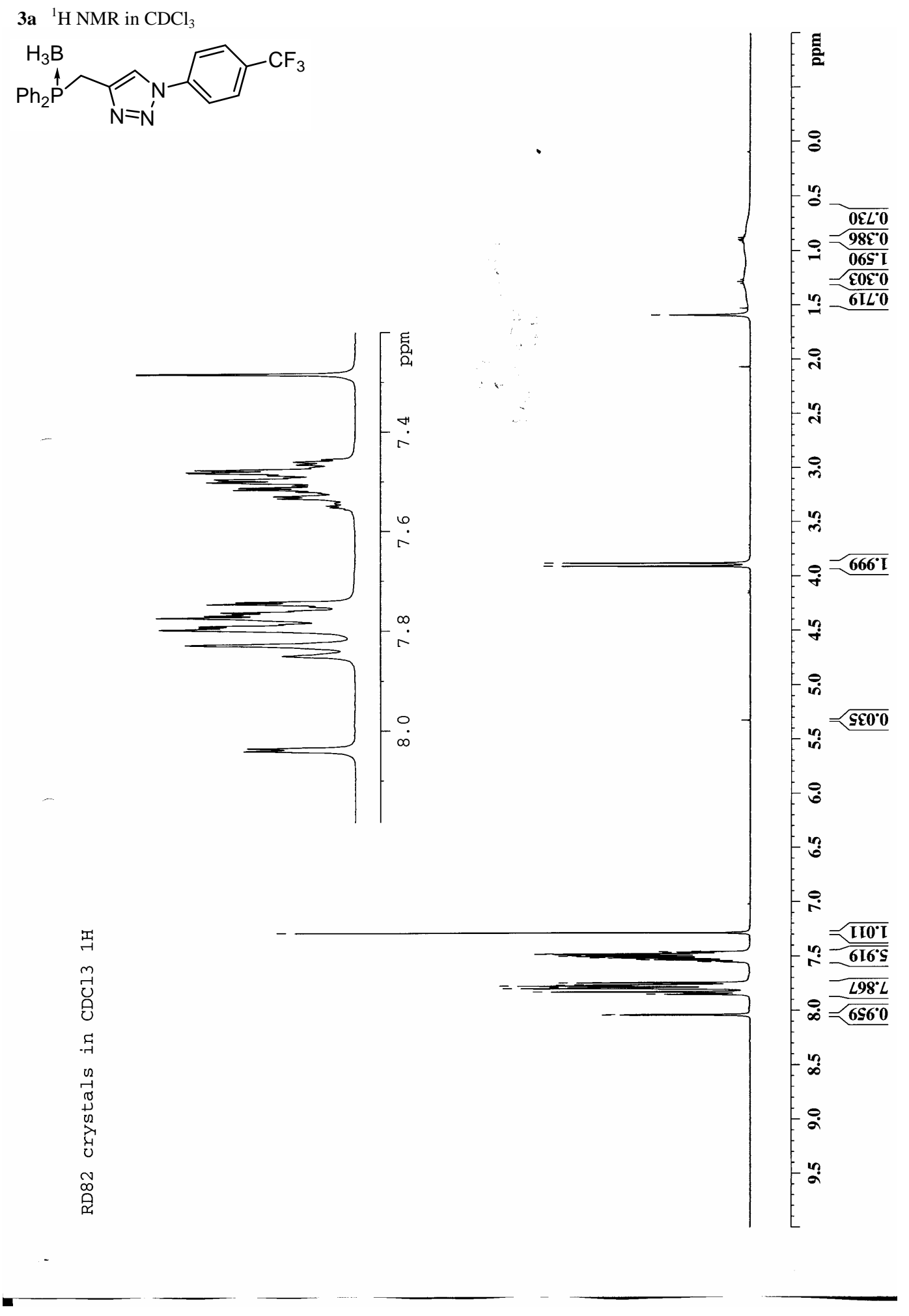


3a ${ }^{13} \mathrm{C} \mathrm{NMR}$ in $\mathrm{CDCl}_{3}$
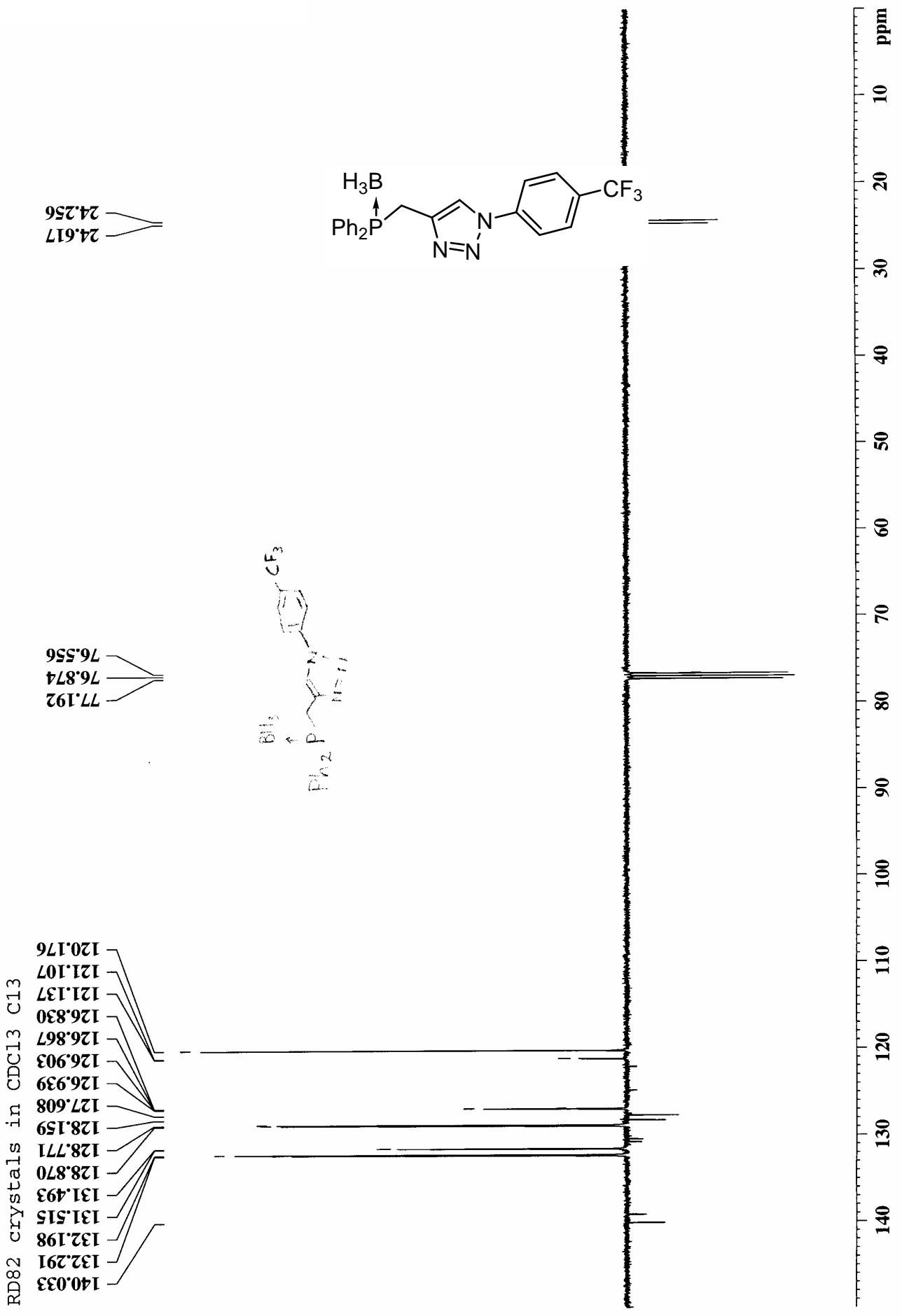


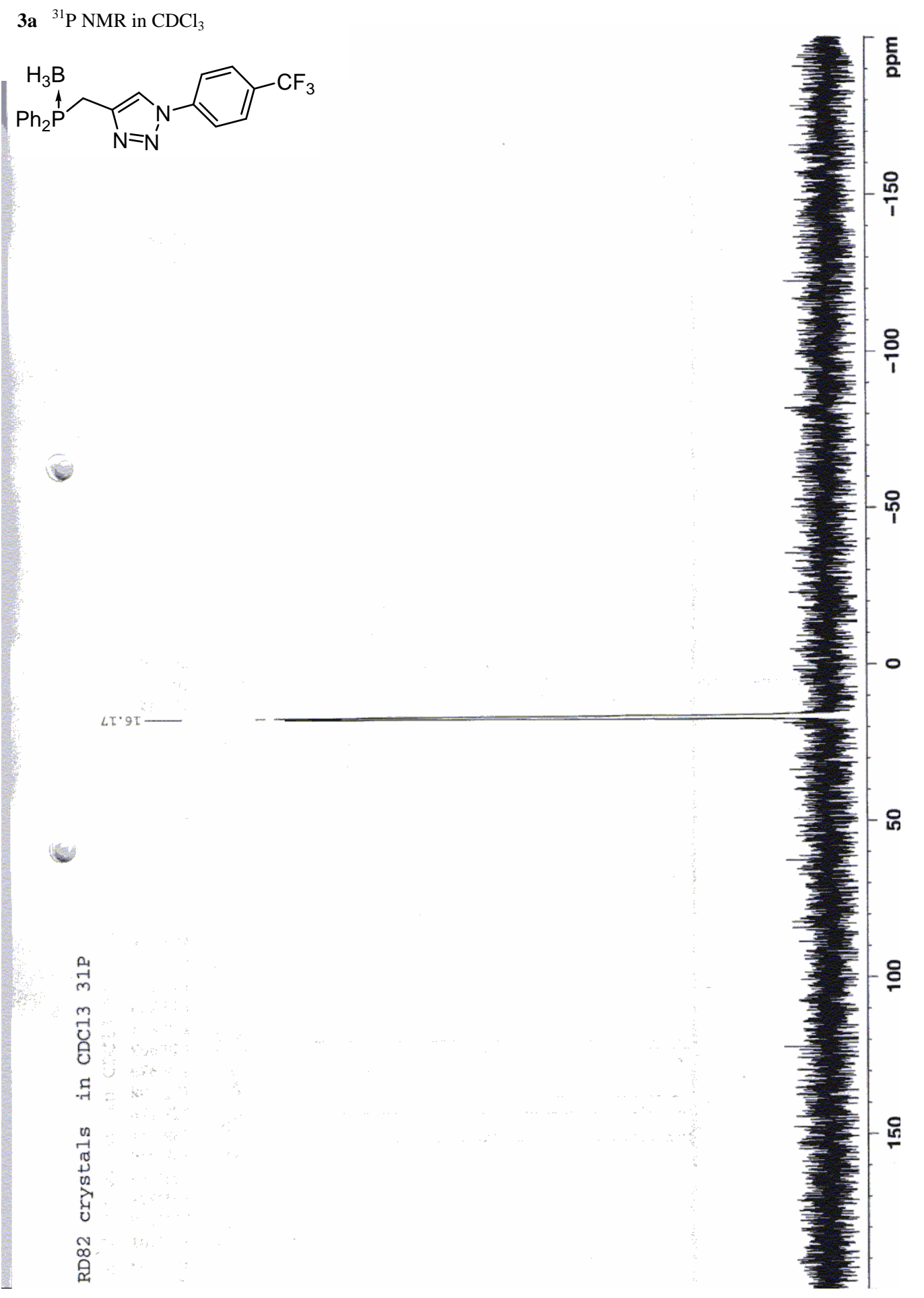




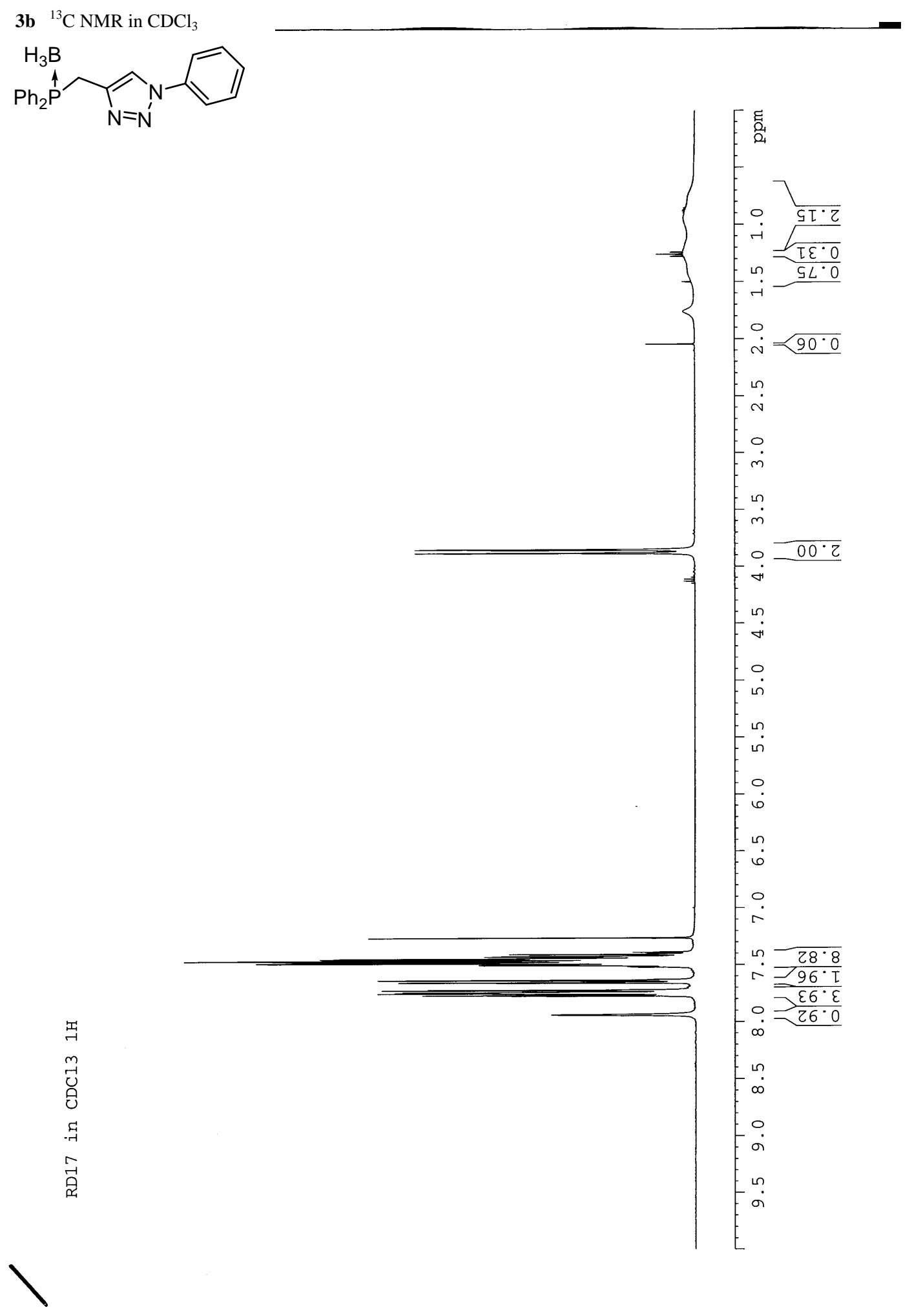


3b ${ }^{1} \mathrm{H} \mathrm{NMR}$ in $\mathrm{CDCl}_{3}$

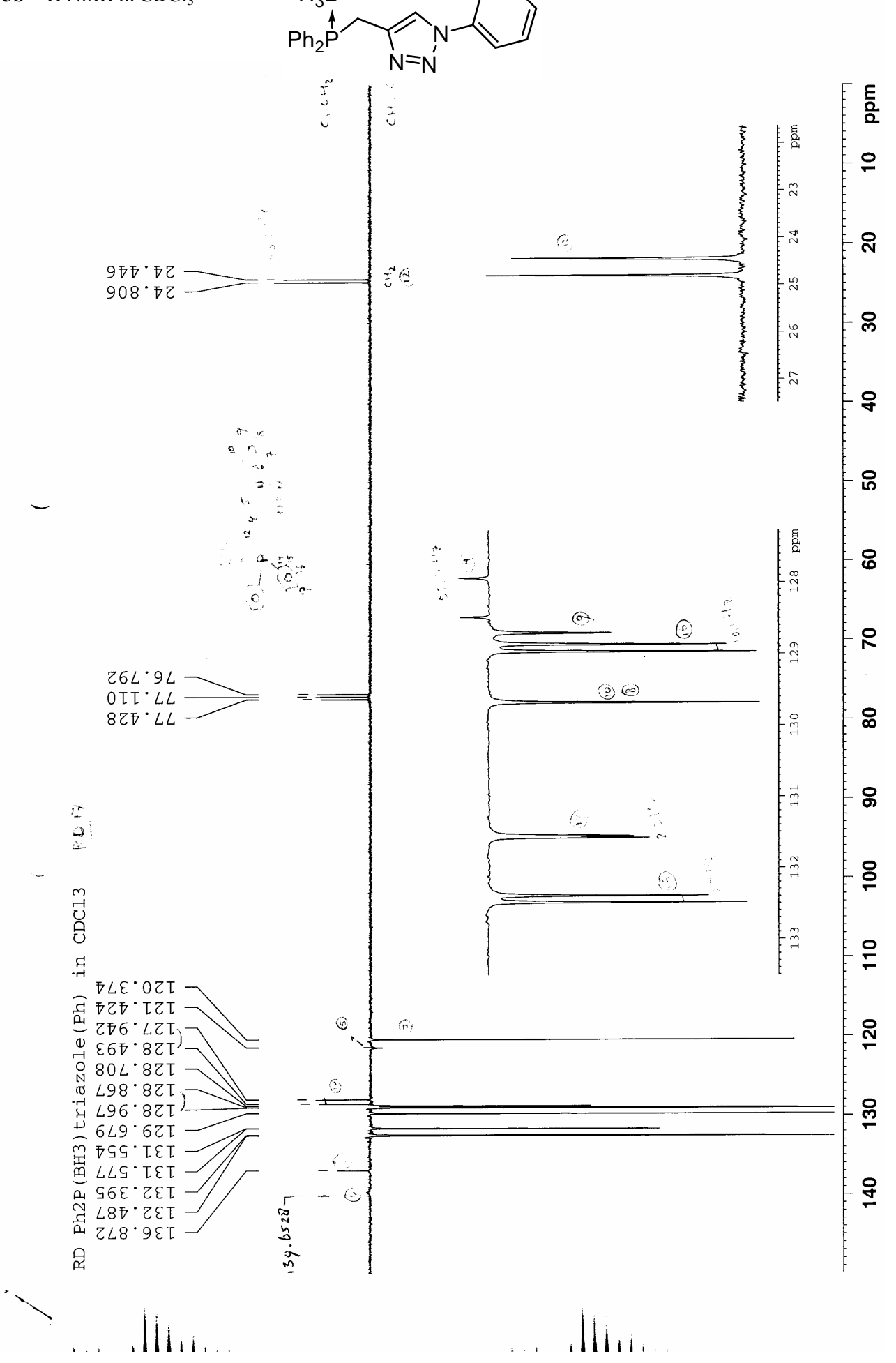




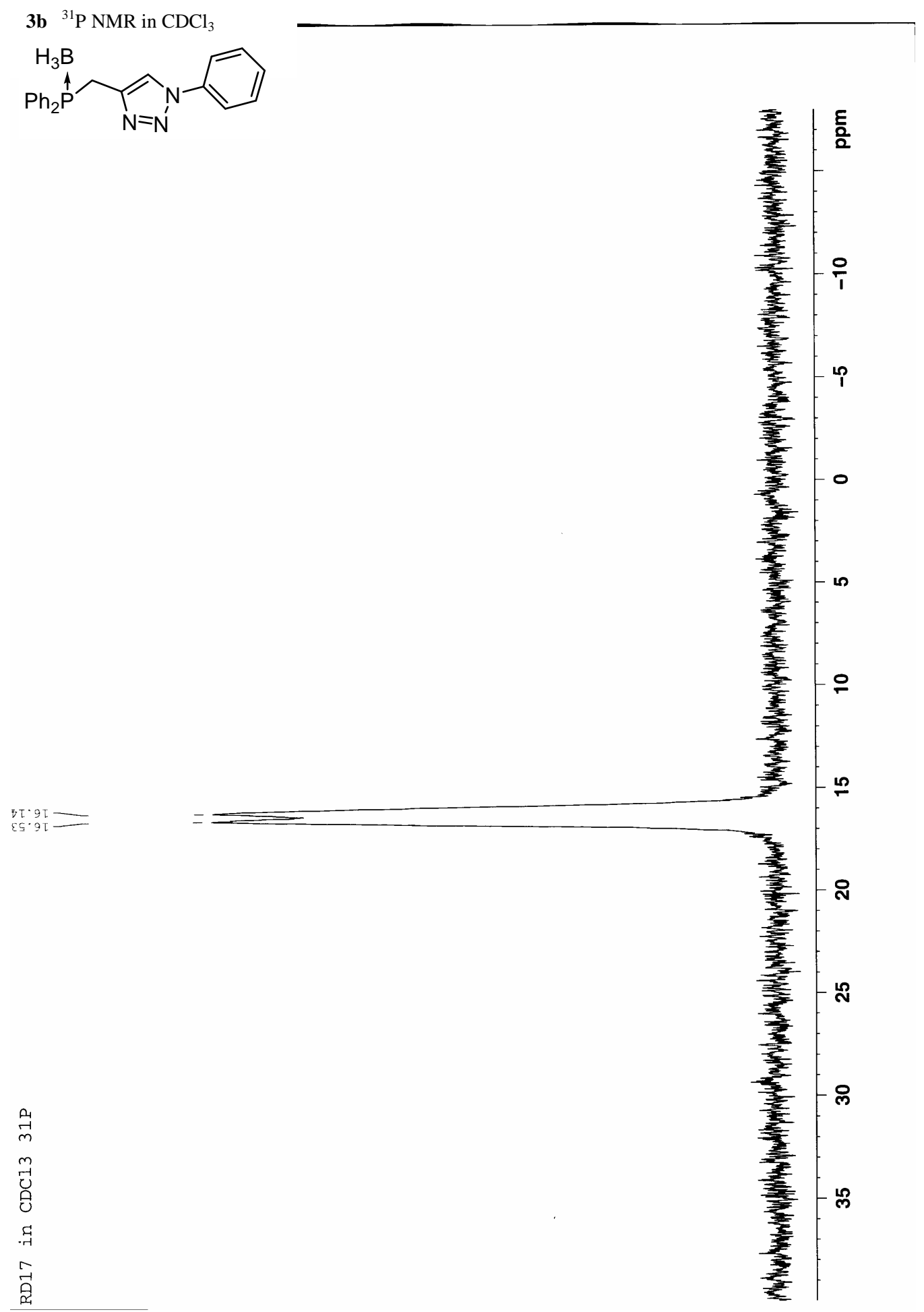




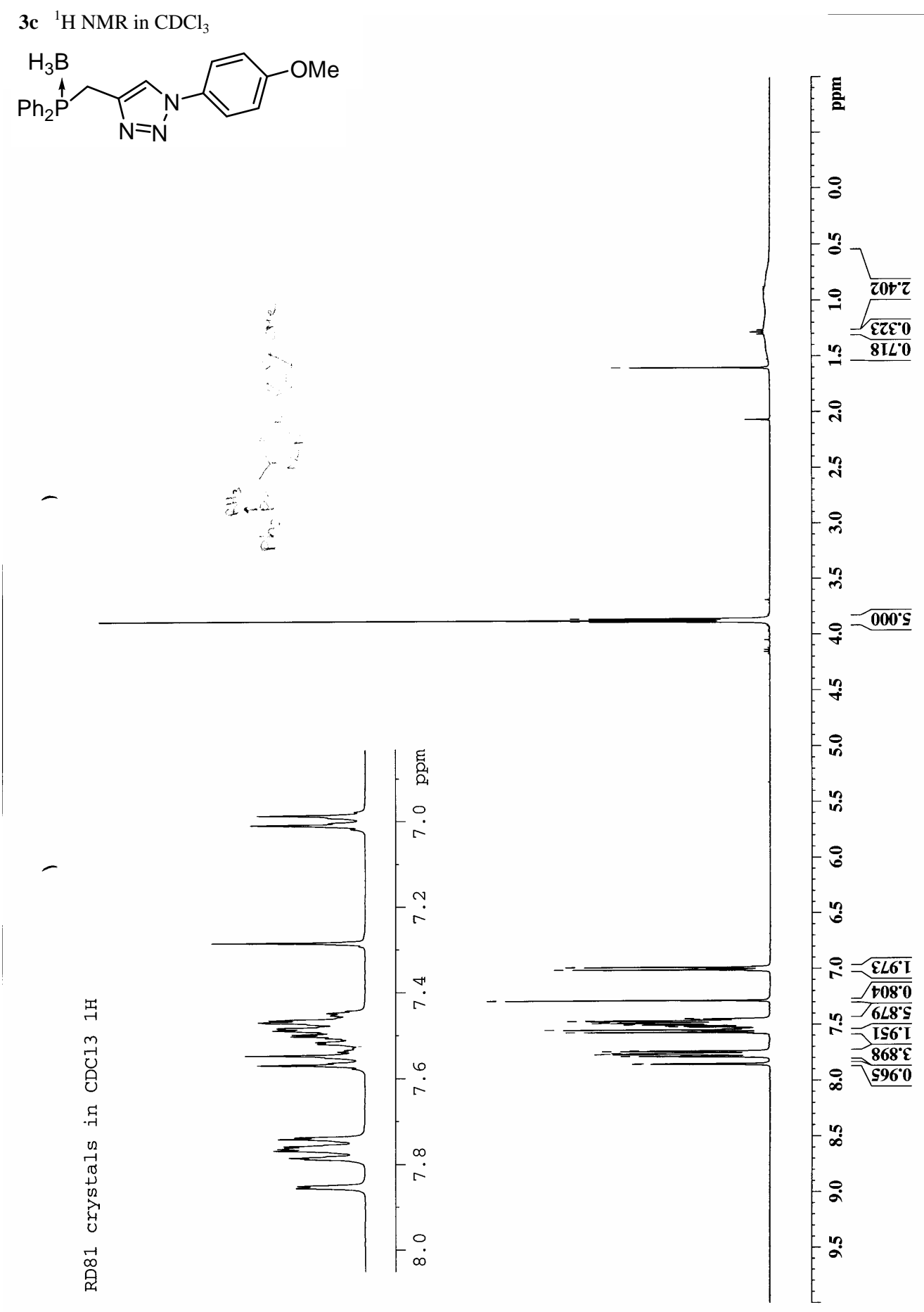




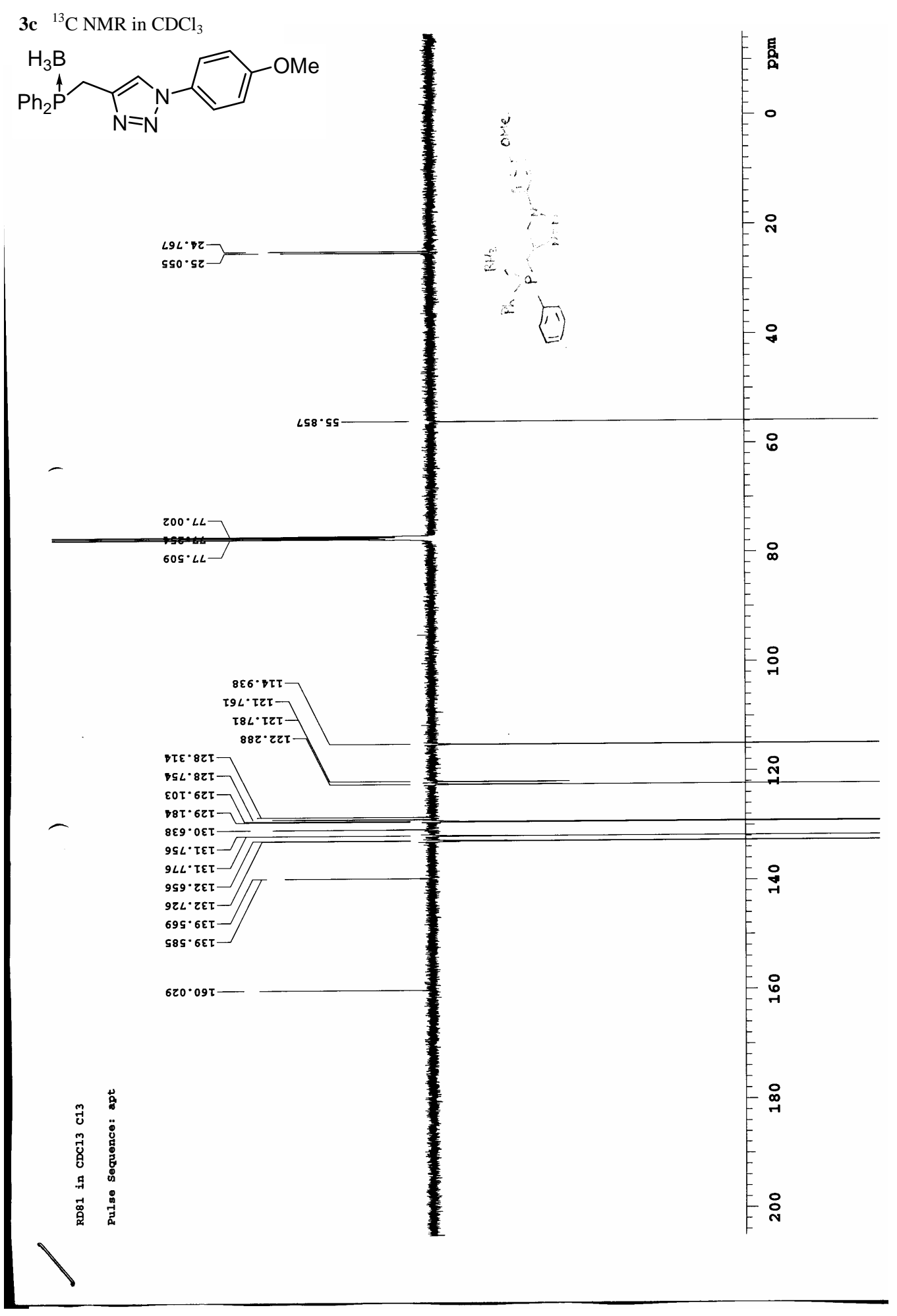




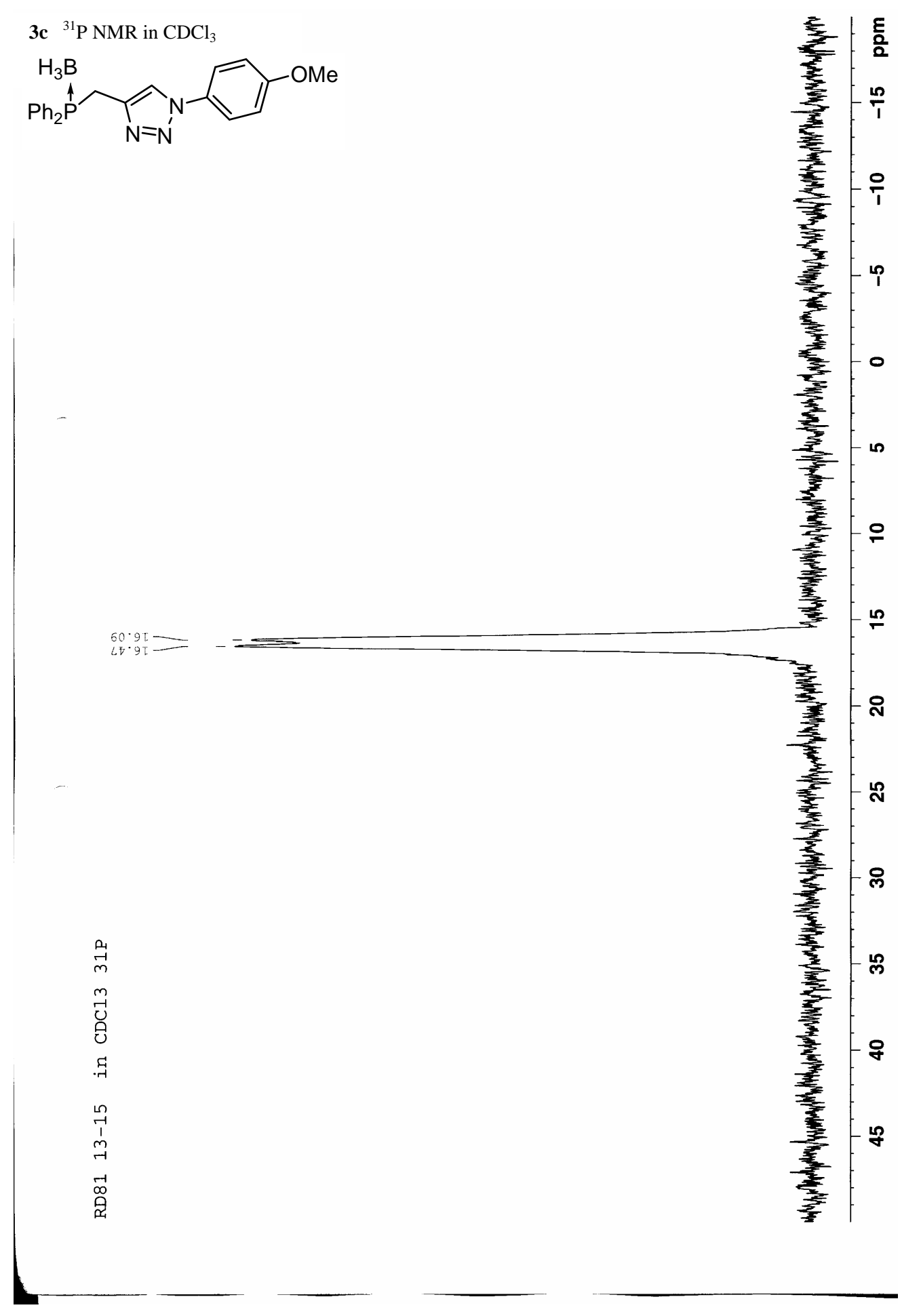




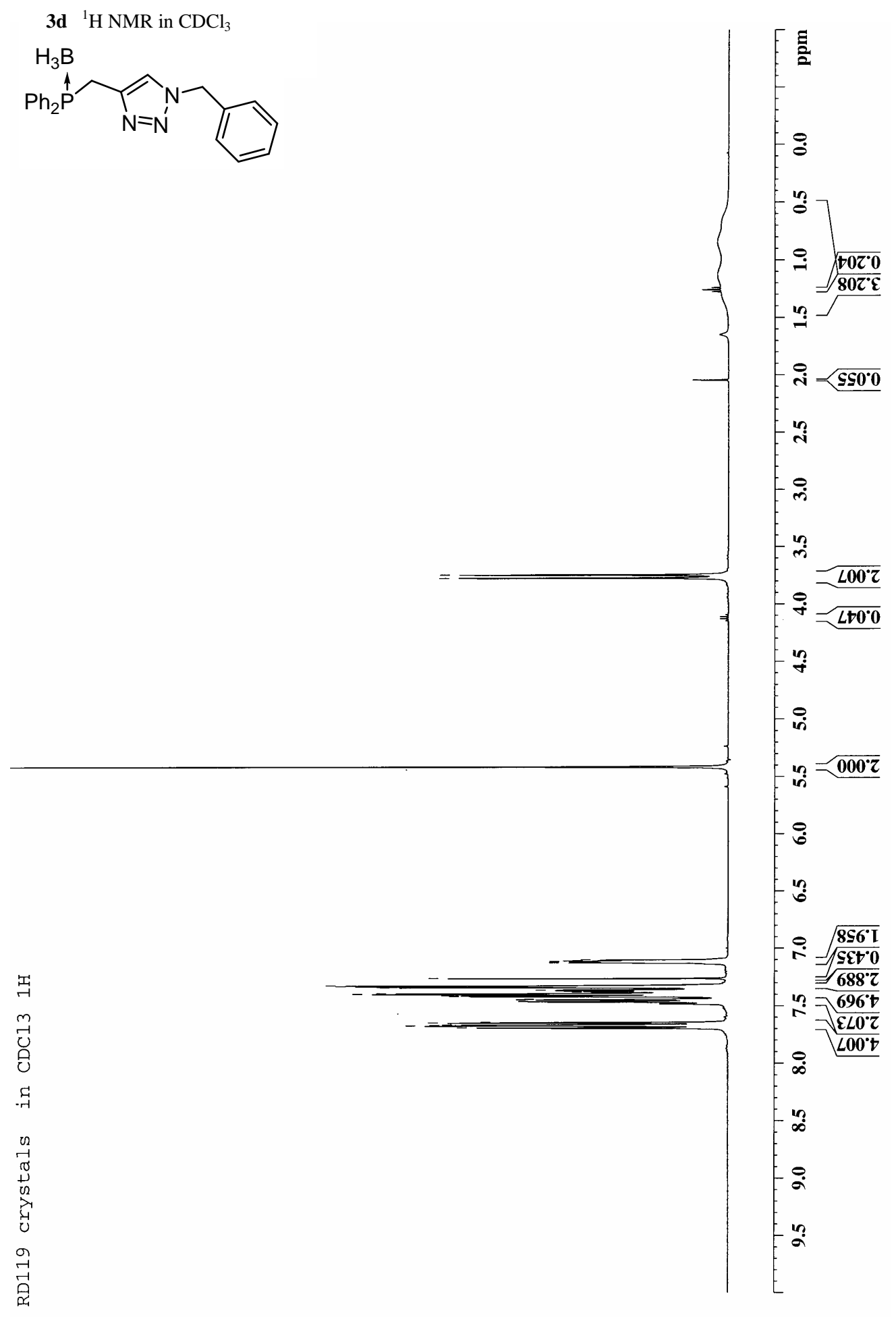




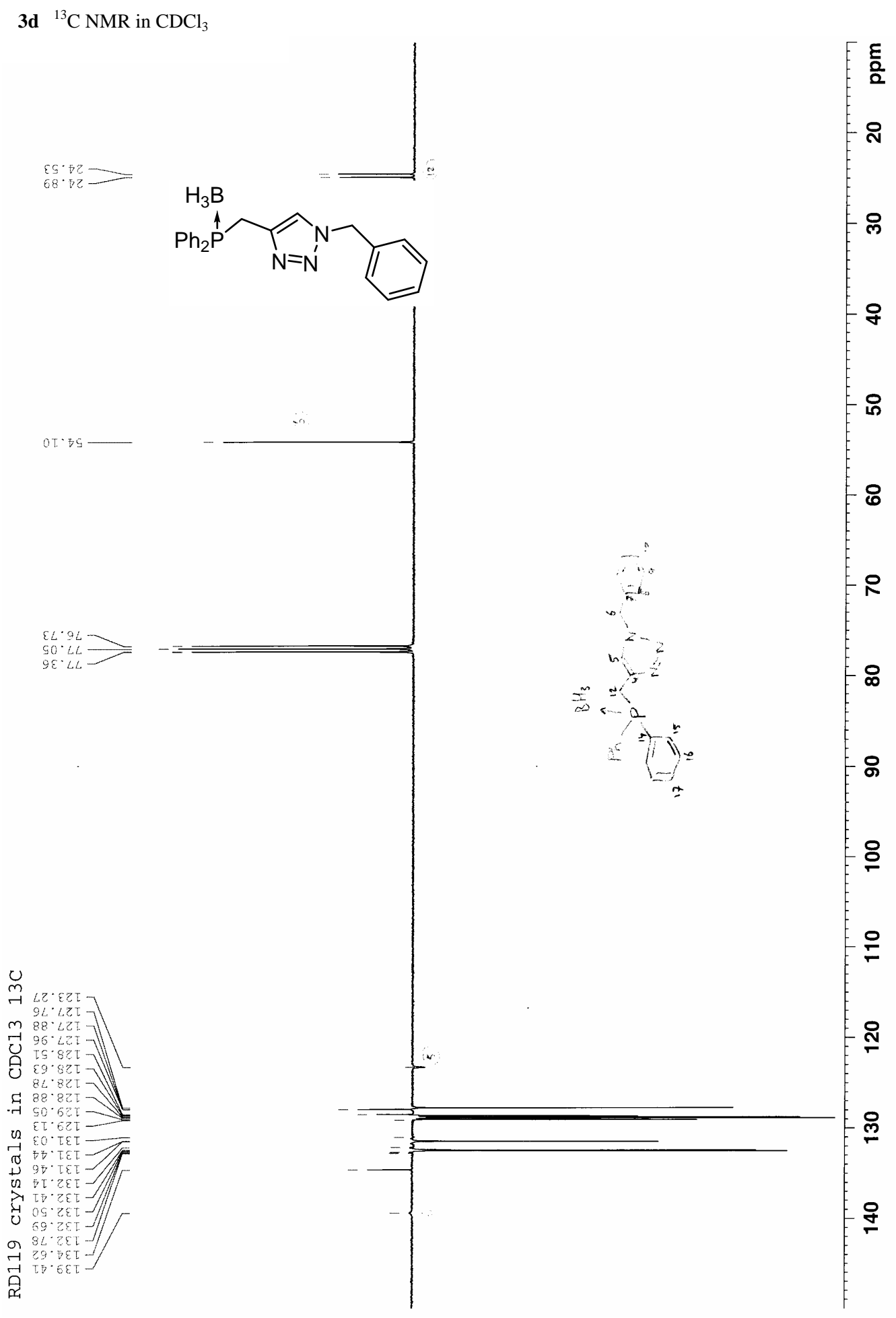




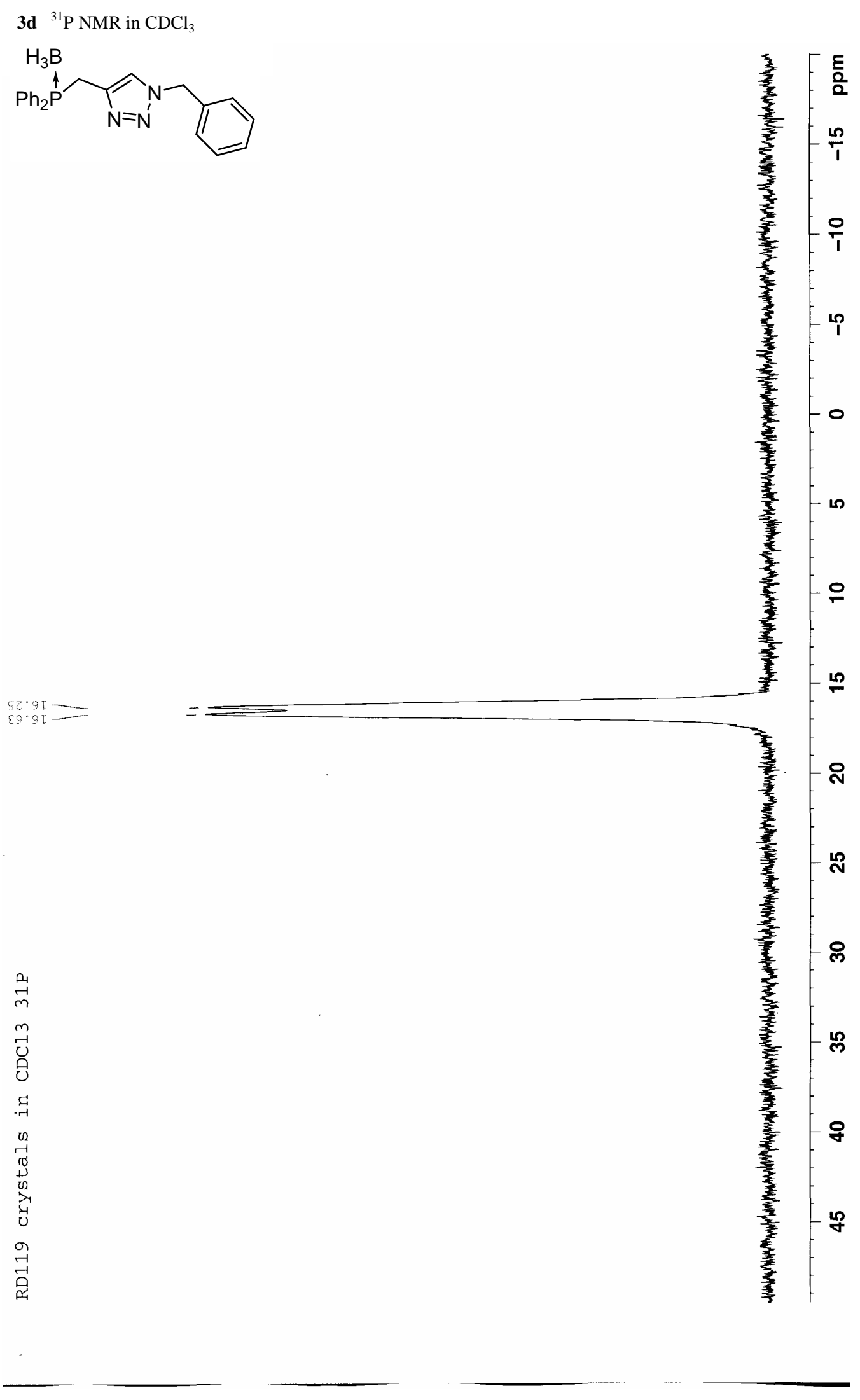


3e ${ }^{1} \mathrm{H} \mathrm{NMR}$ in $\mathrm{CDCl}_{3}$

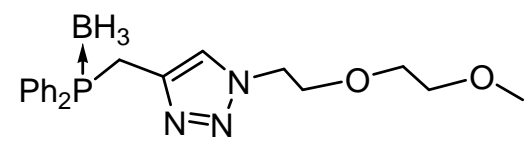

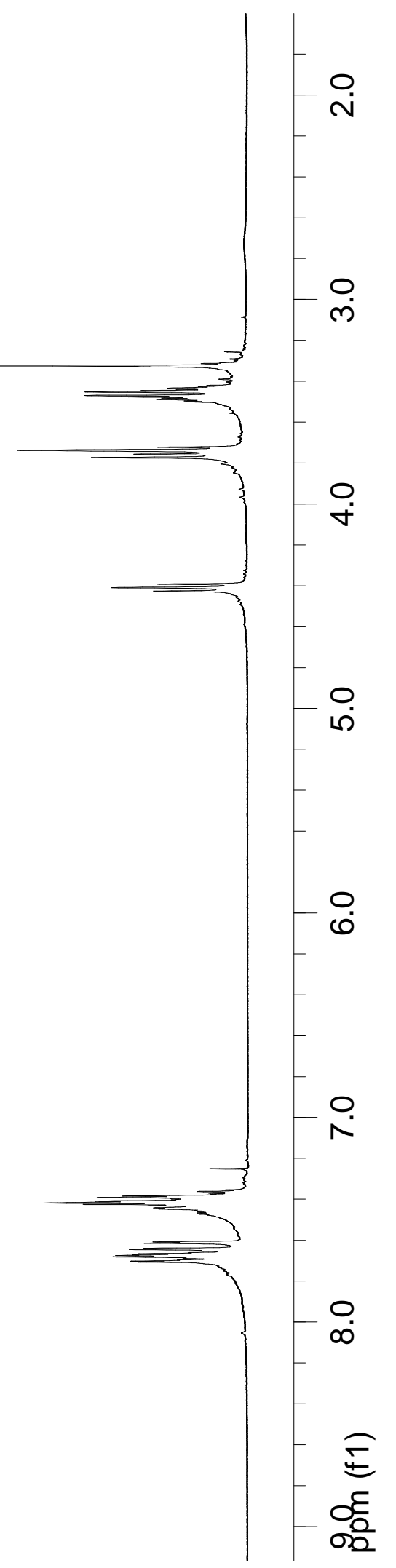




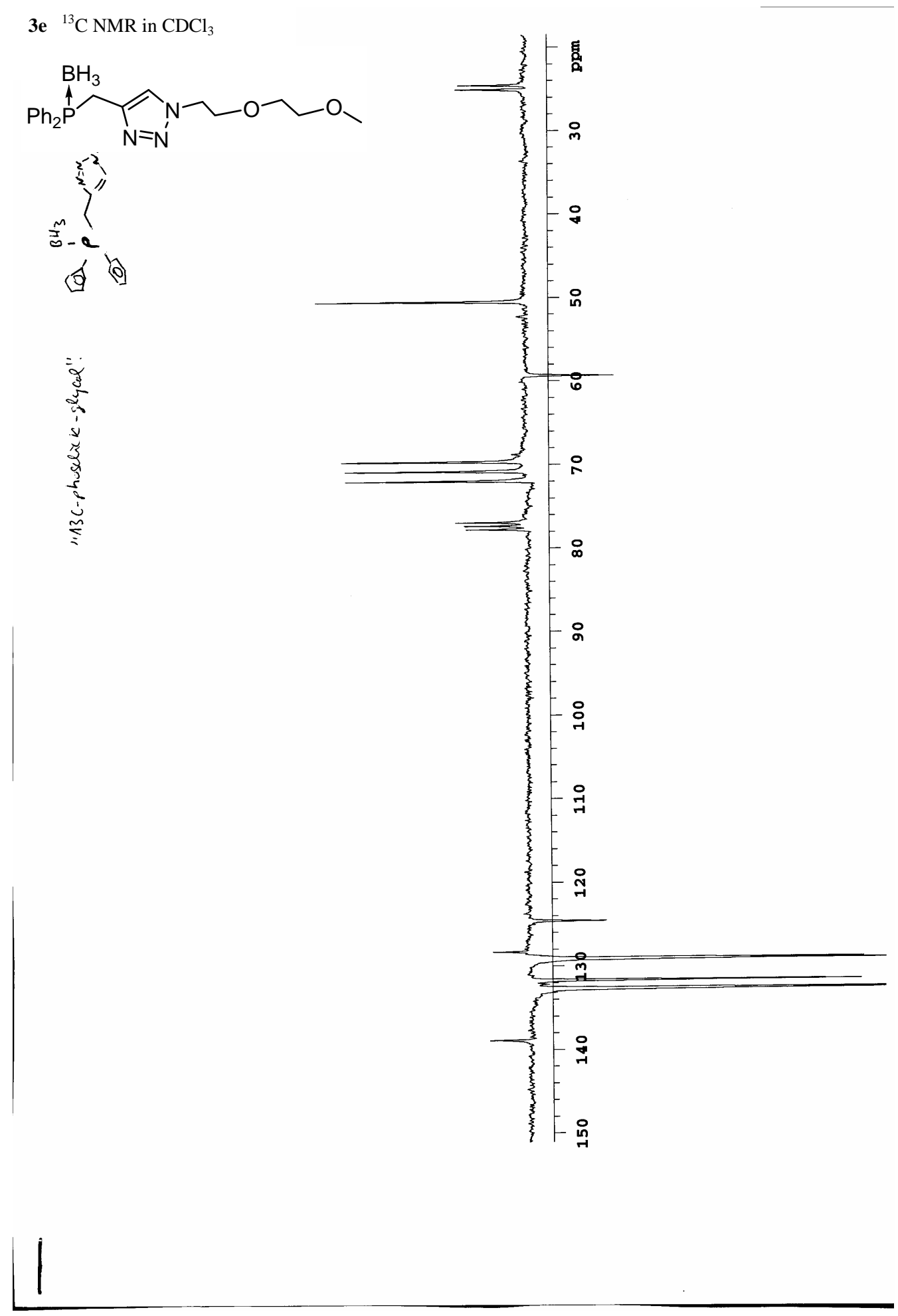




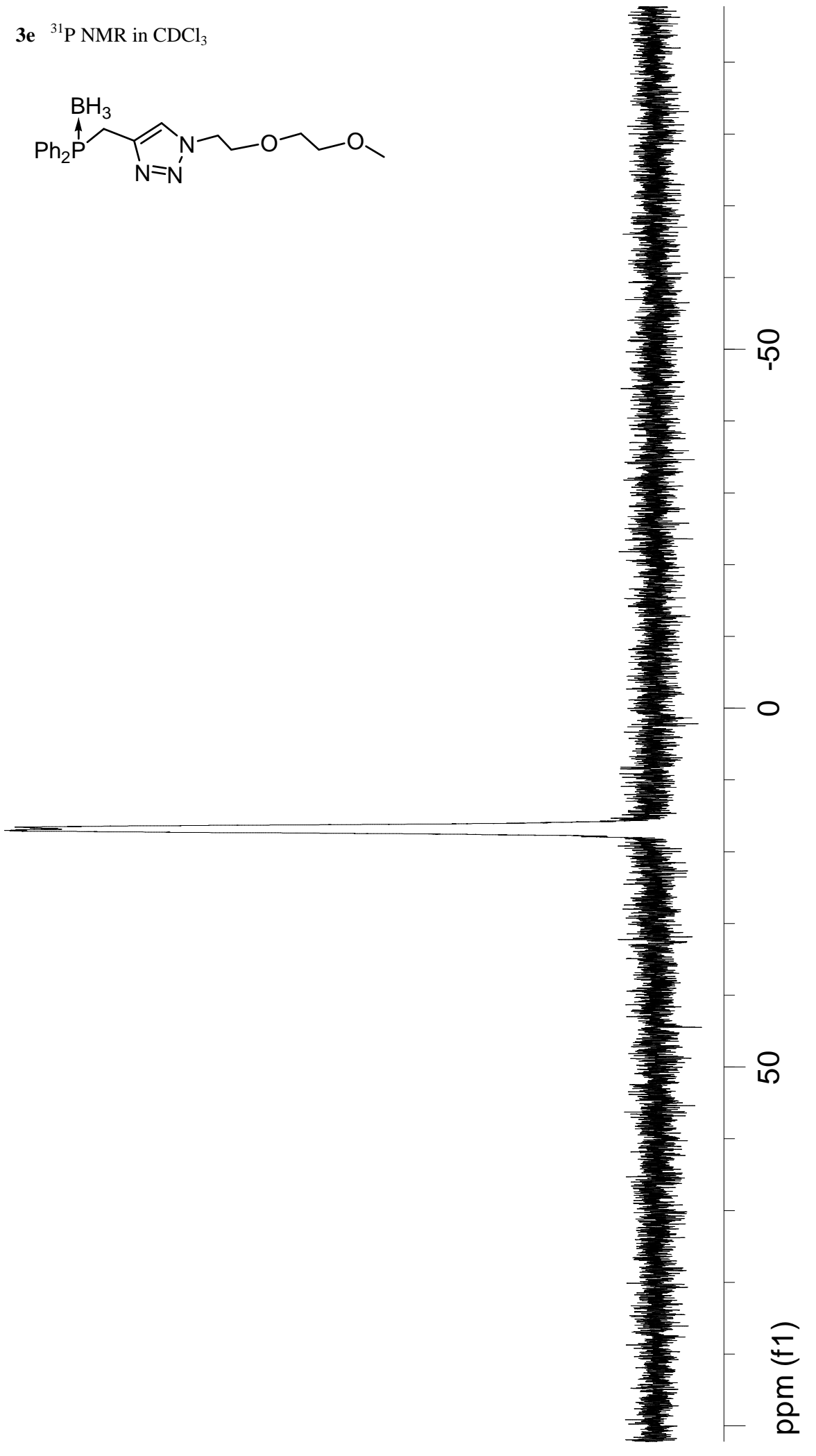


4a ${ }^{1} \mathrm{H} \mathrm{NMR}$ in $\mathrm{CDCl}_{3}$
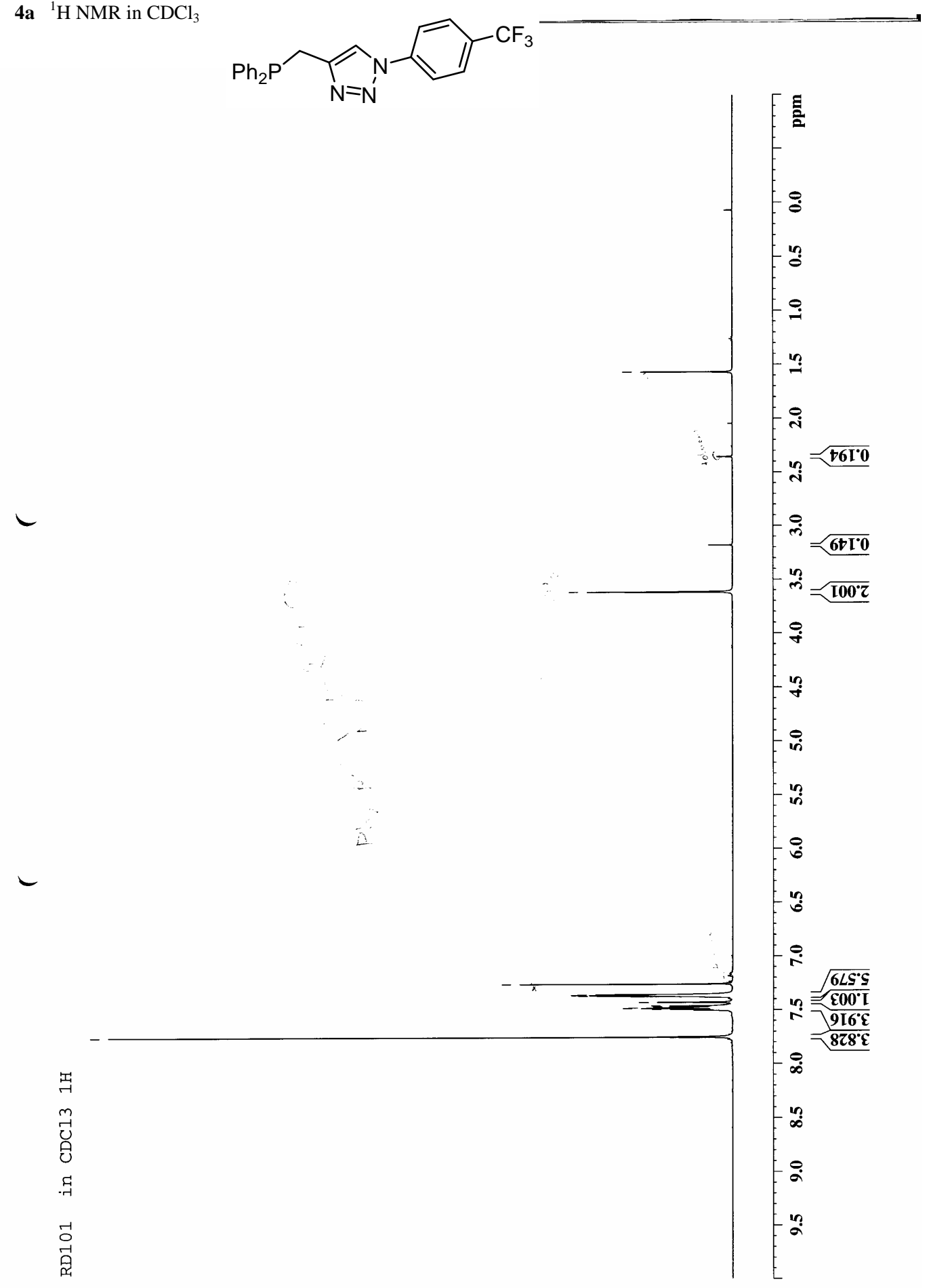
1 


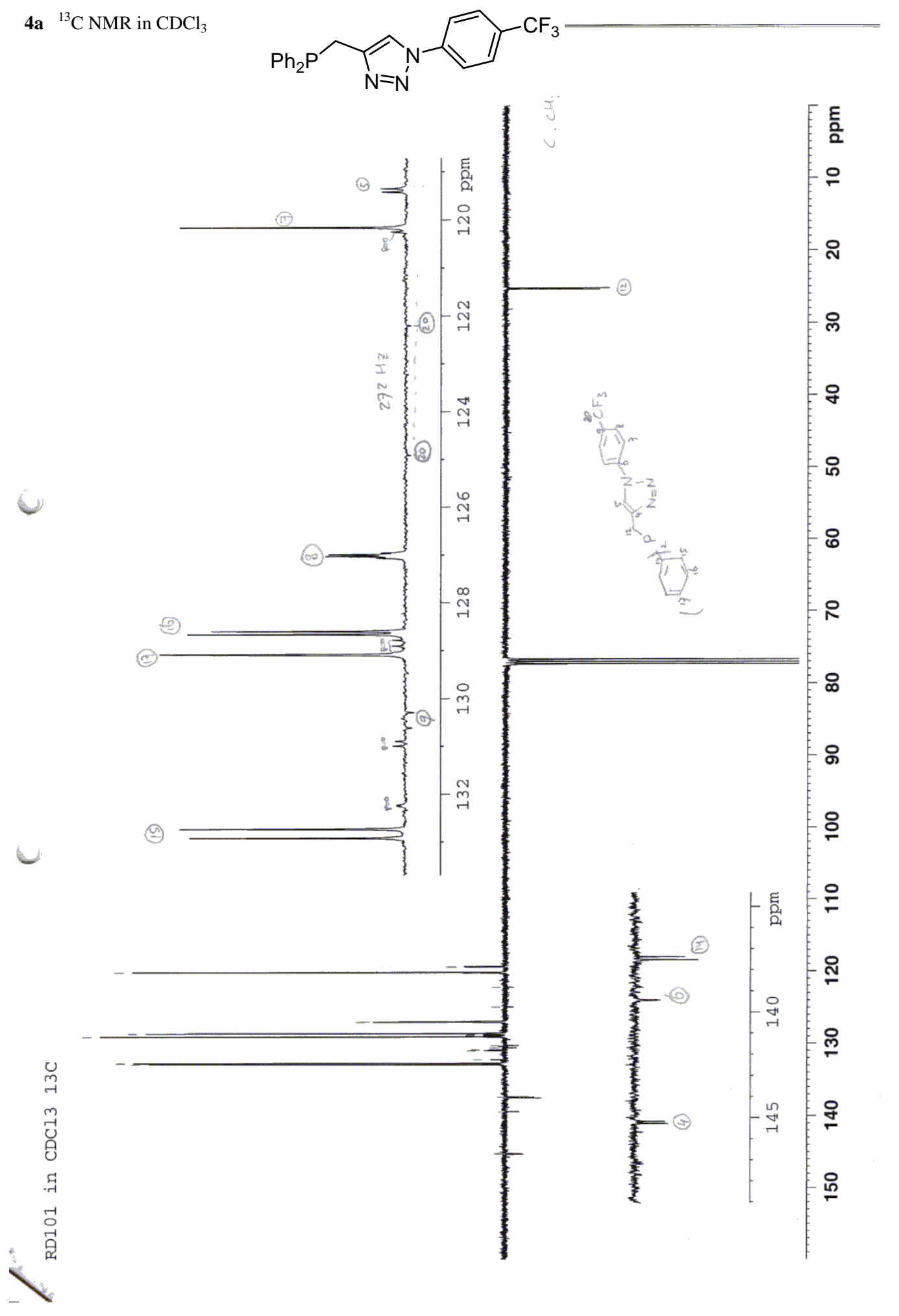




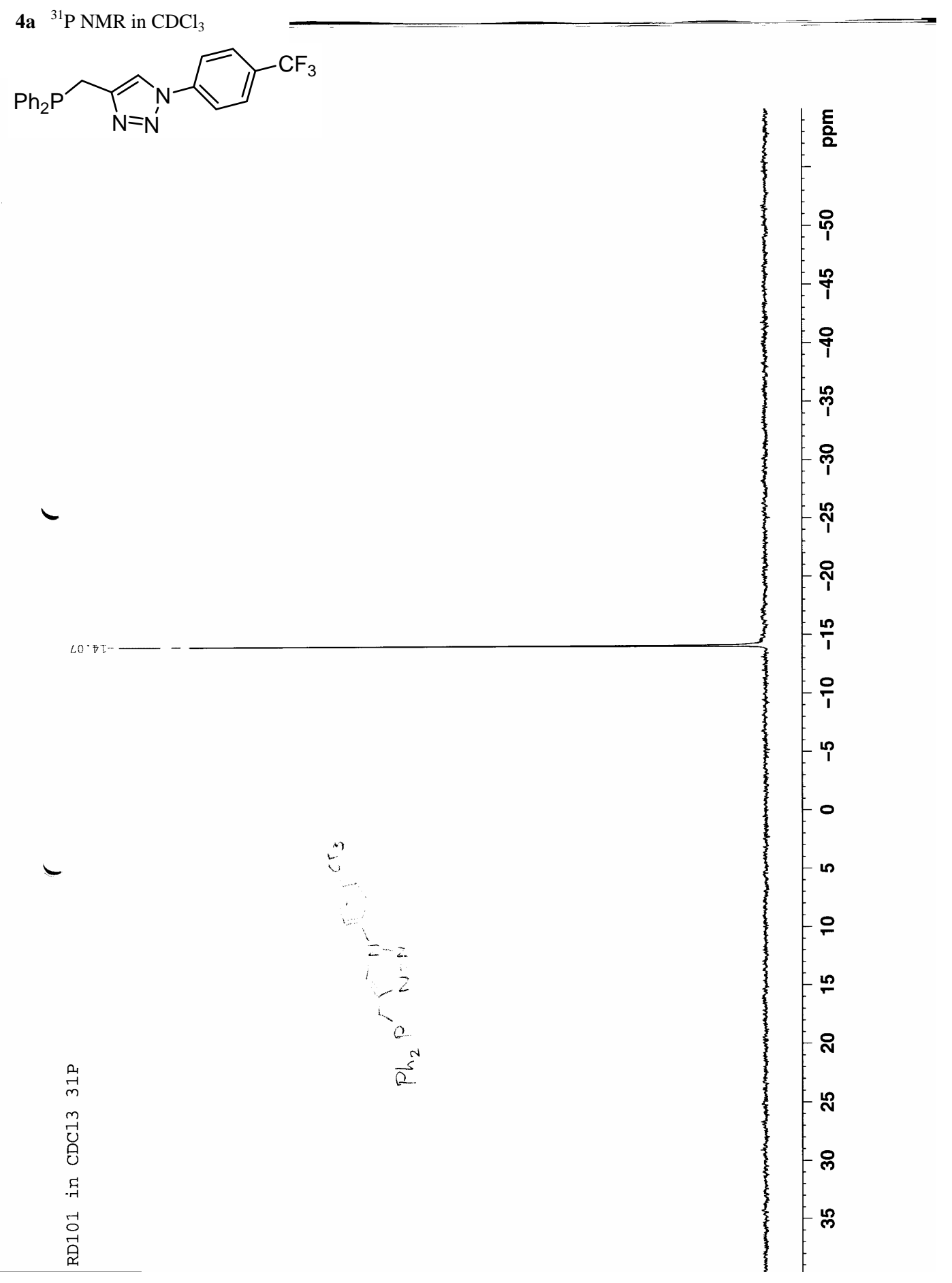


4b ${ }^{1} \mathrm{H} \mathrm{NMR}$ in $\mathrm{CDCl}_{3}$<smiles>PPCc1cn(-c2ccccc2)nn1</smiles>

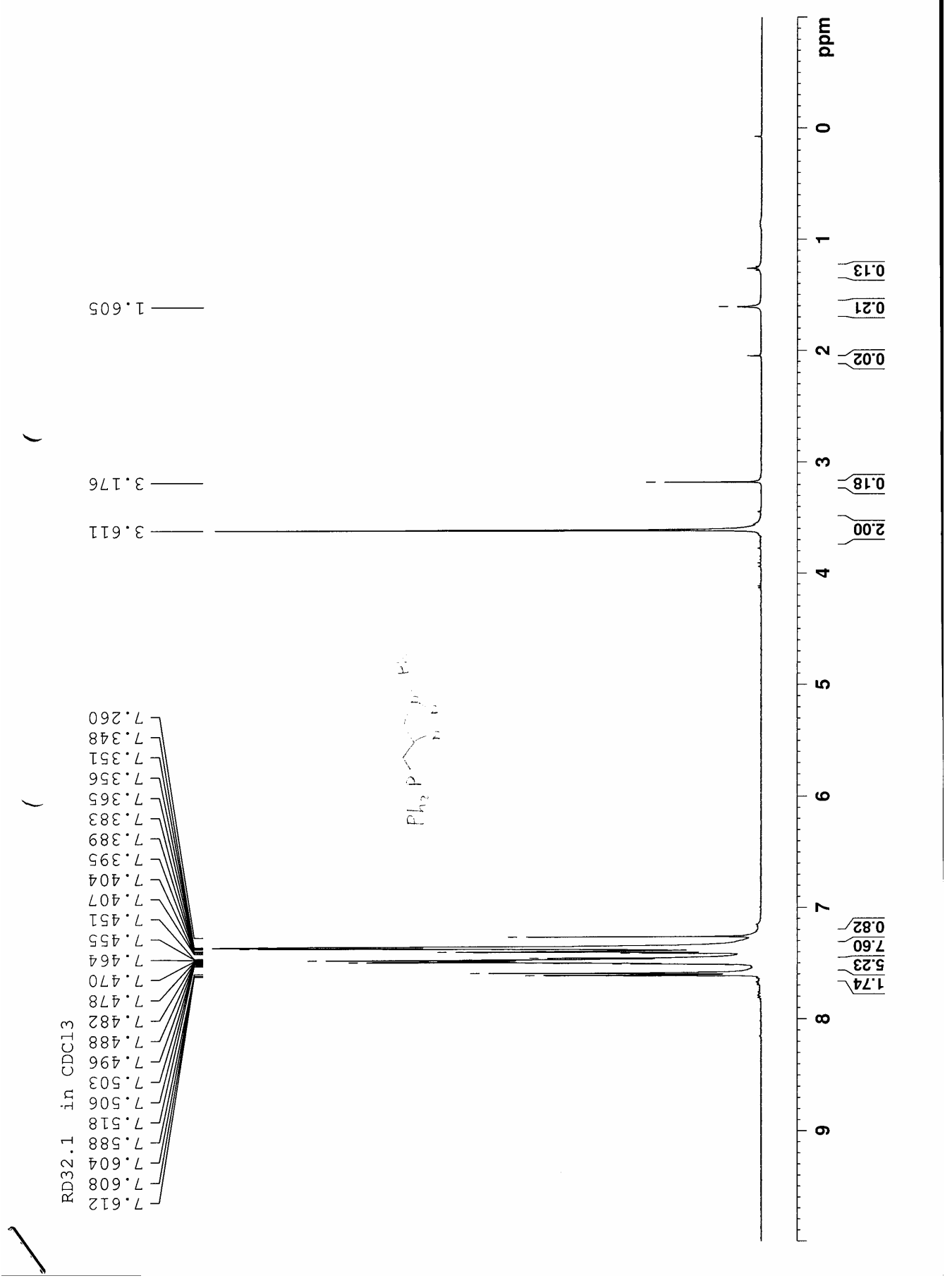


4b ${ }^{13} \mathrm{C}$ NMR in $\mathrm{CDCl}_{3}$<smiles>PCc1cn(-c2ccccc2)nn1</smiles>

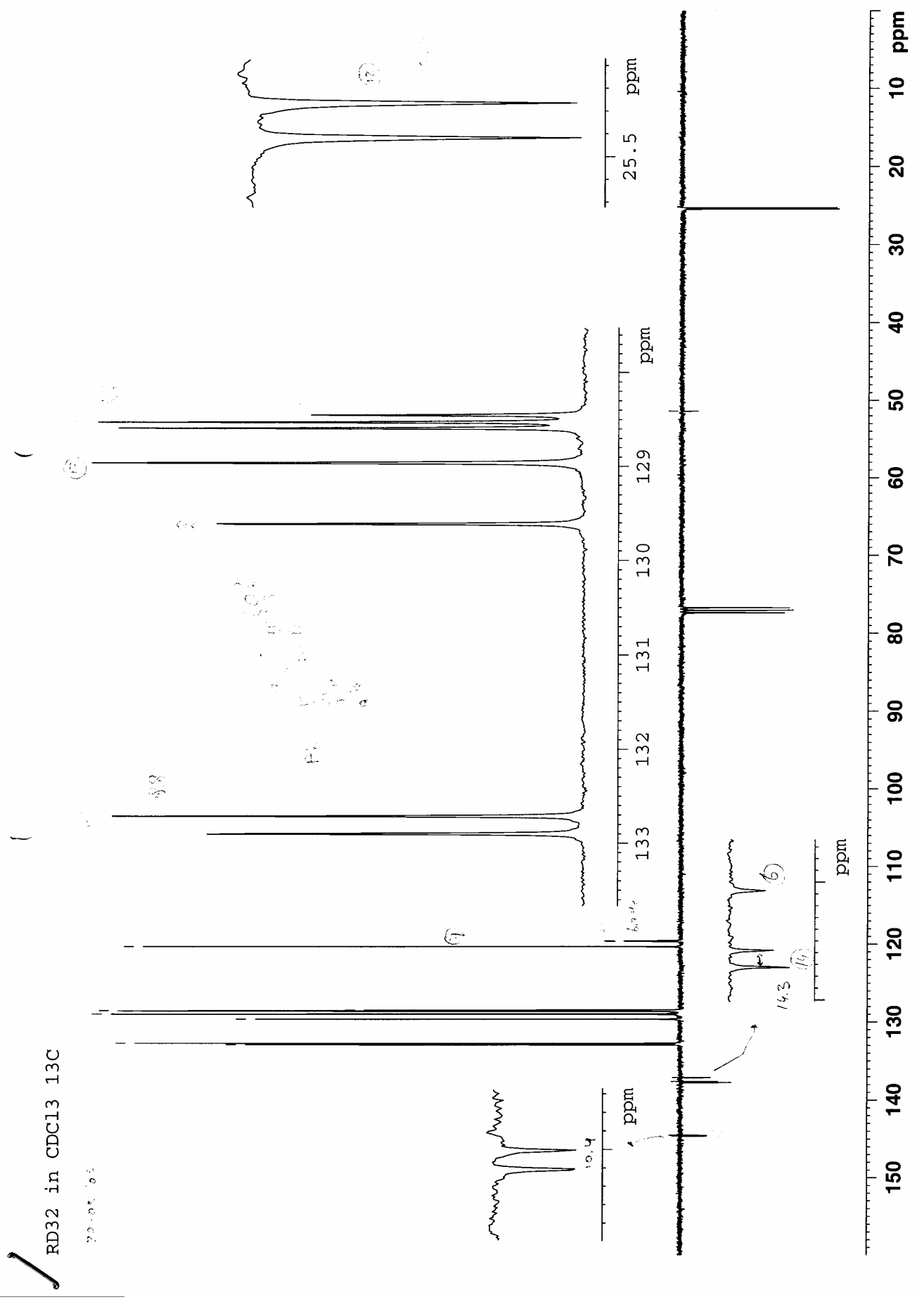




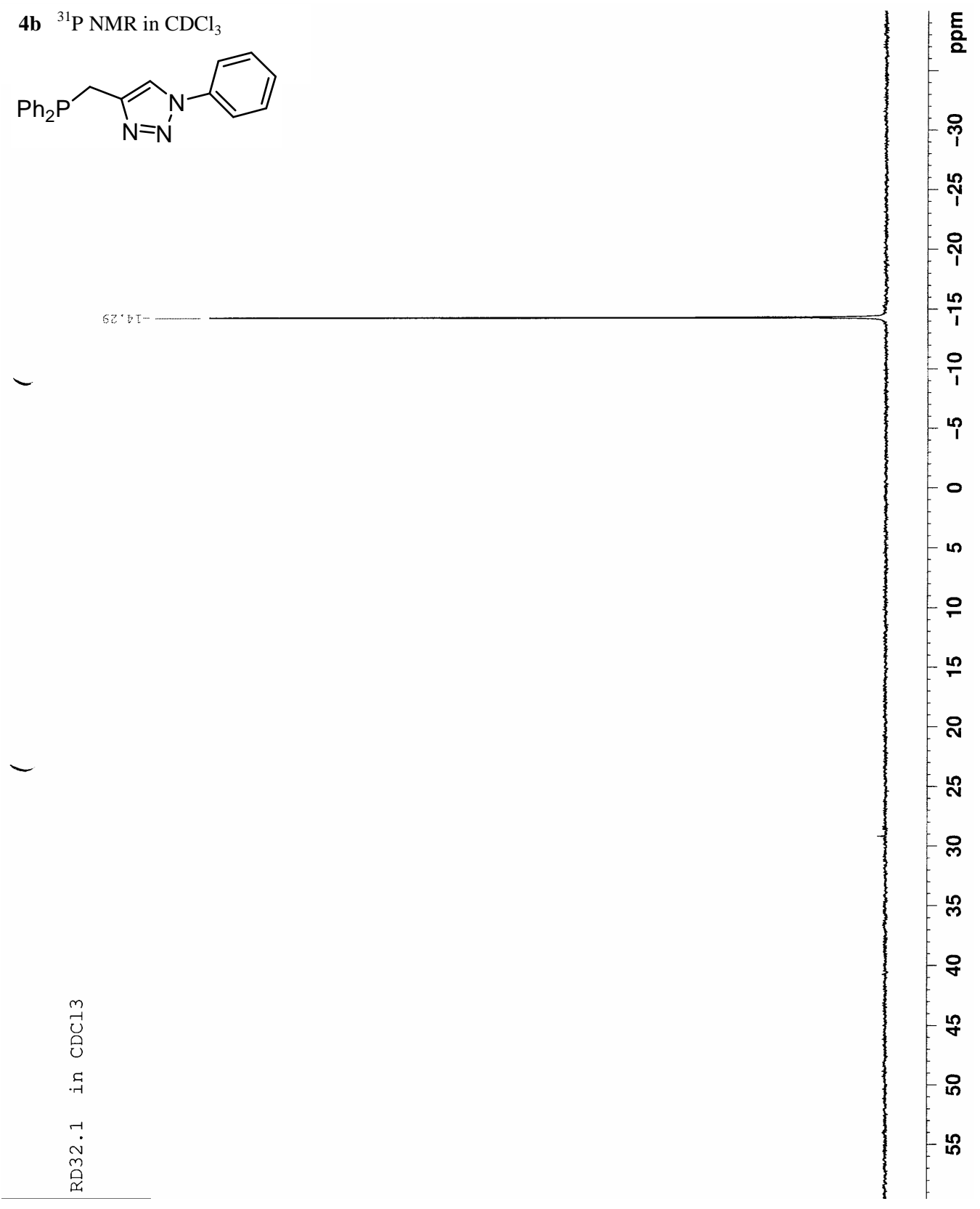




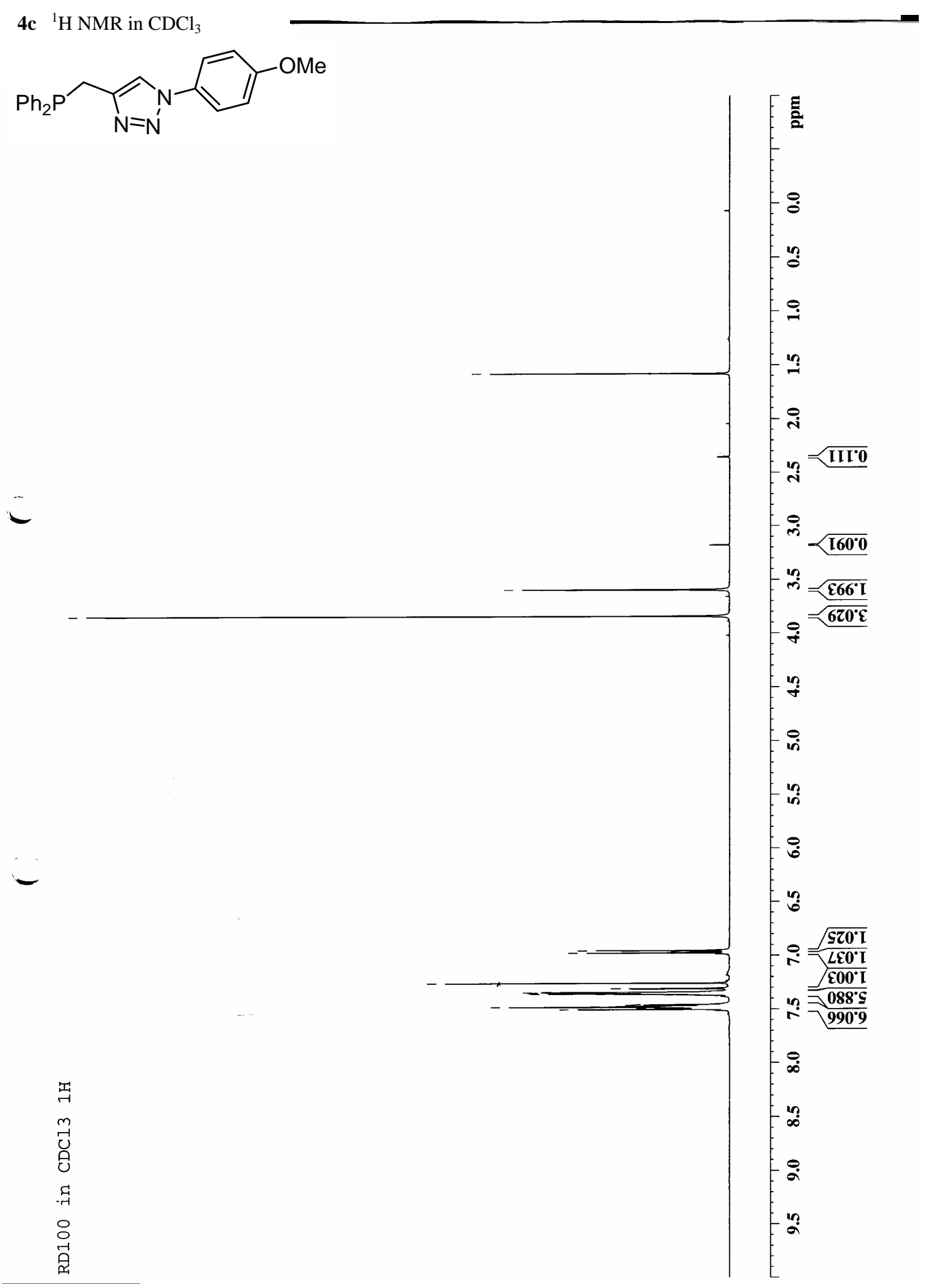




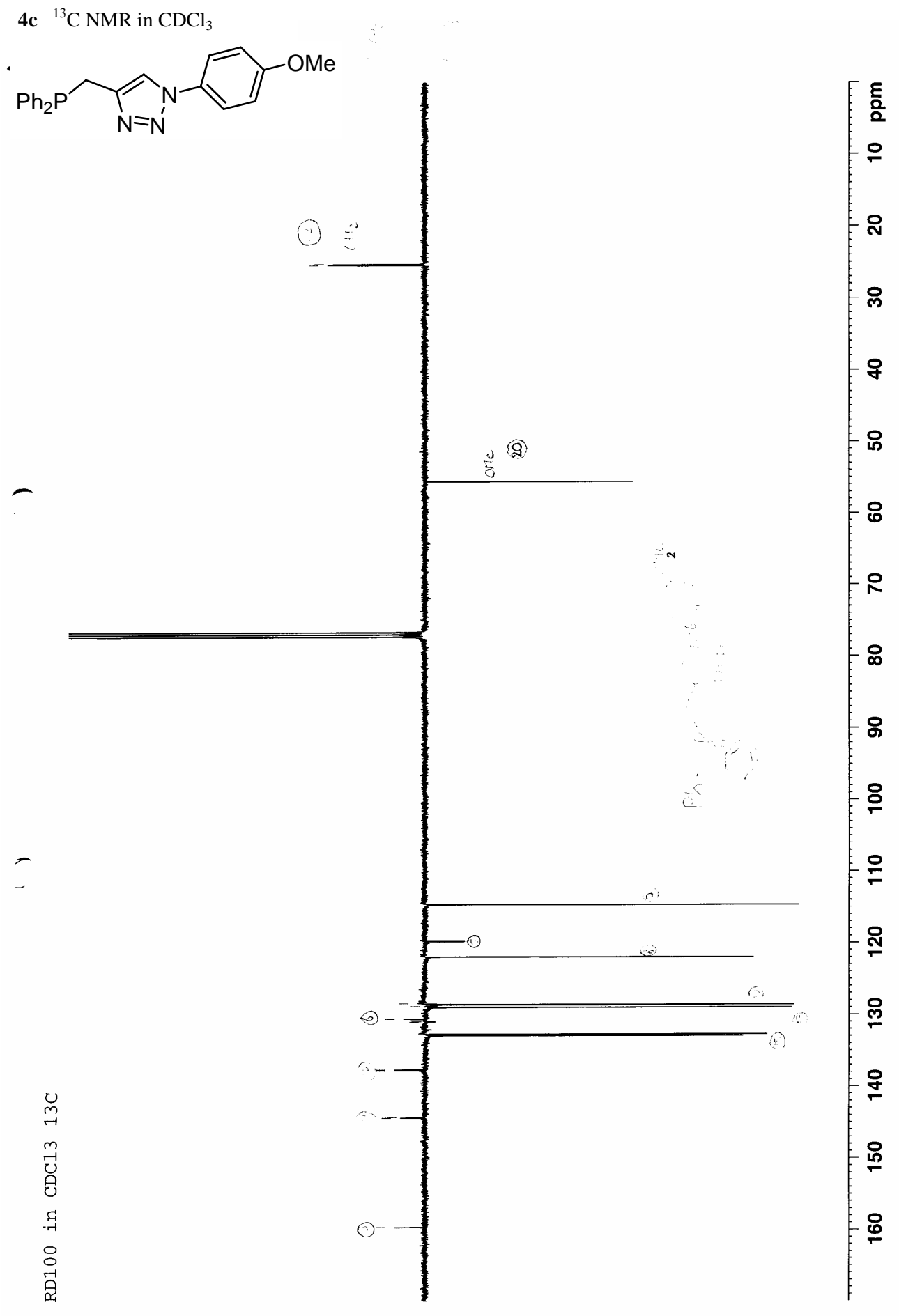




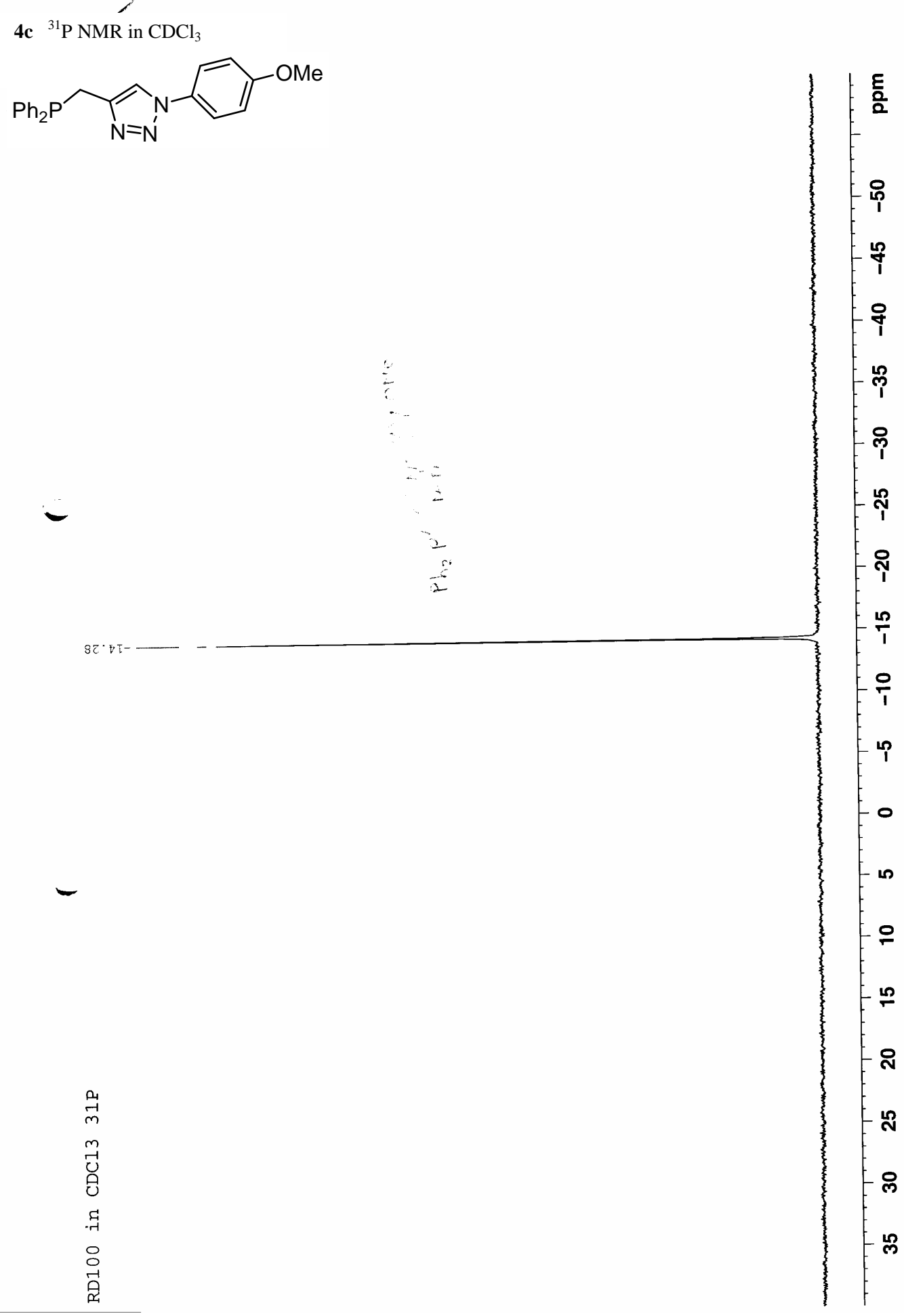


1

4d ${ }^{1} \mathrm{H}$ NMR in $\mathrm{CDCl}_{3}$<smiles>[PbH3]PCc1cn(Cc2ccccc2)nn1</smiles>

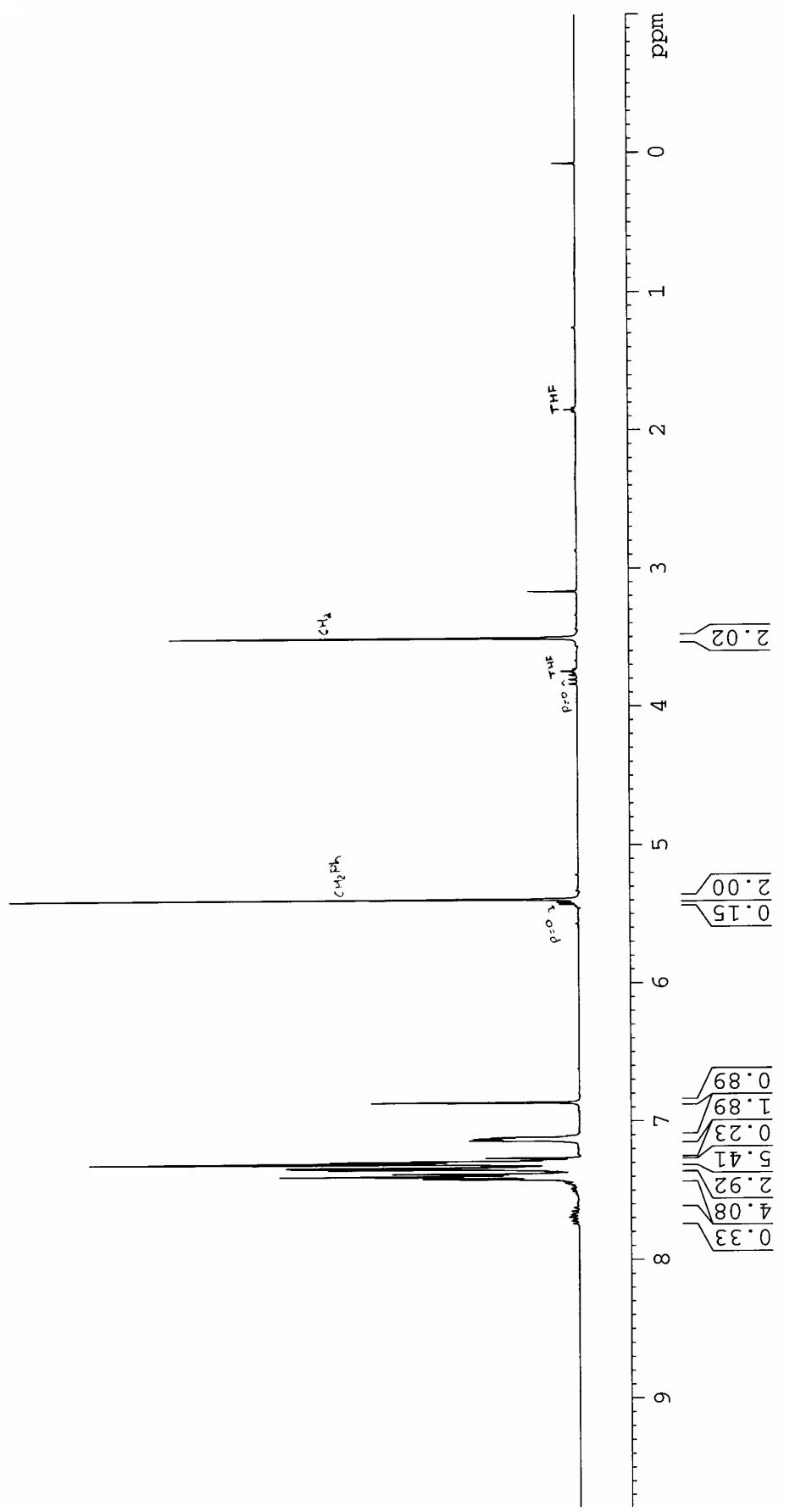

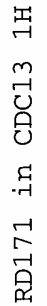




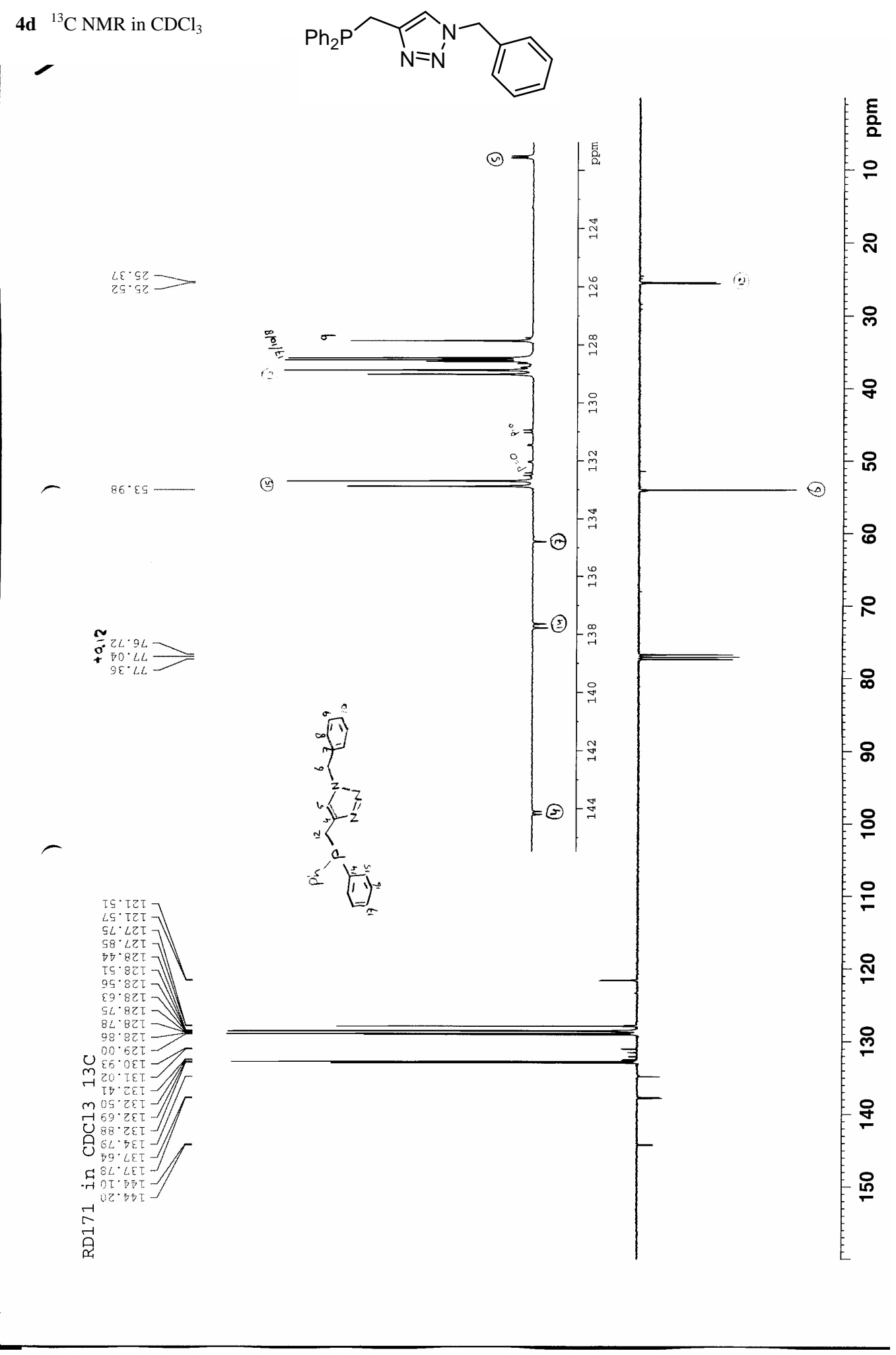




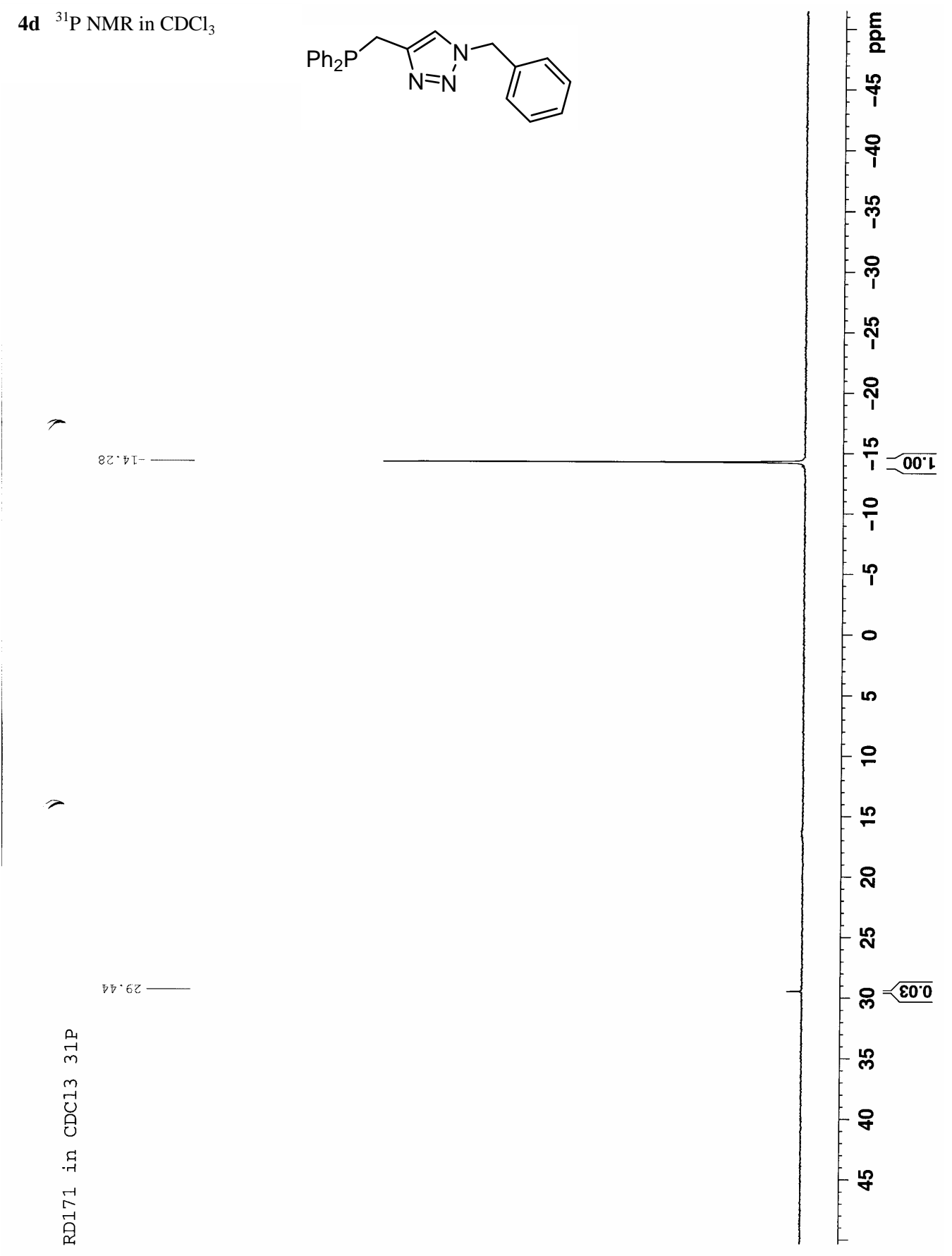




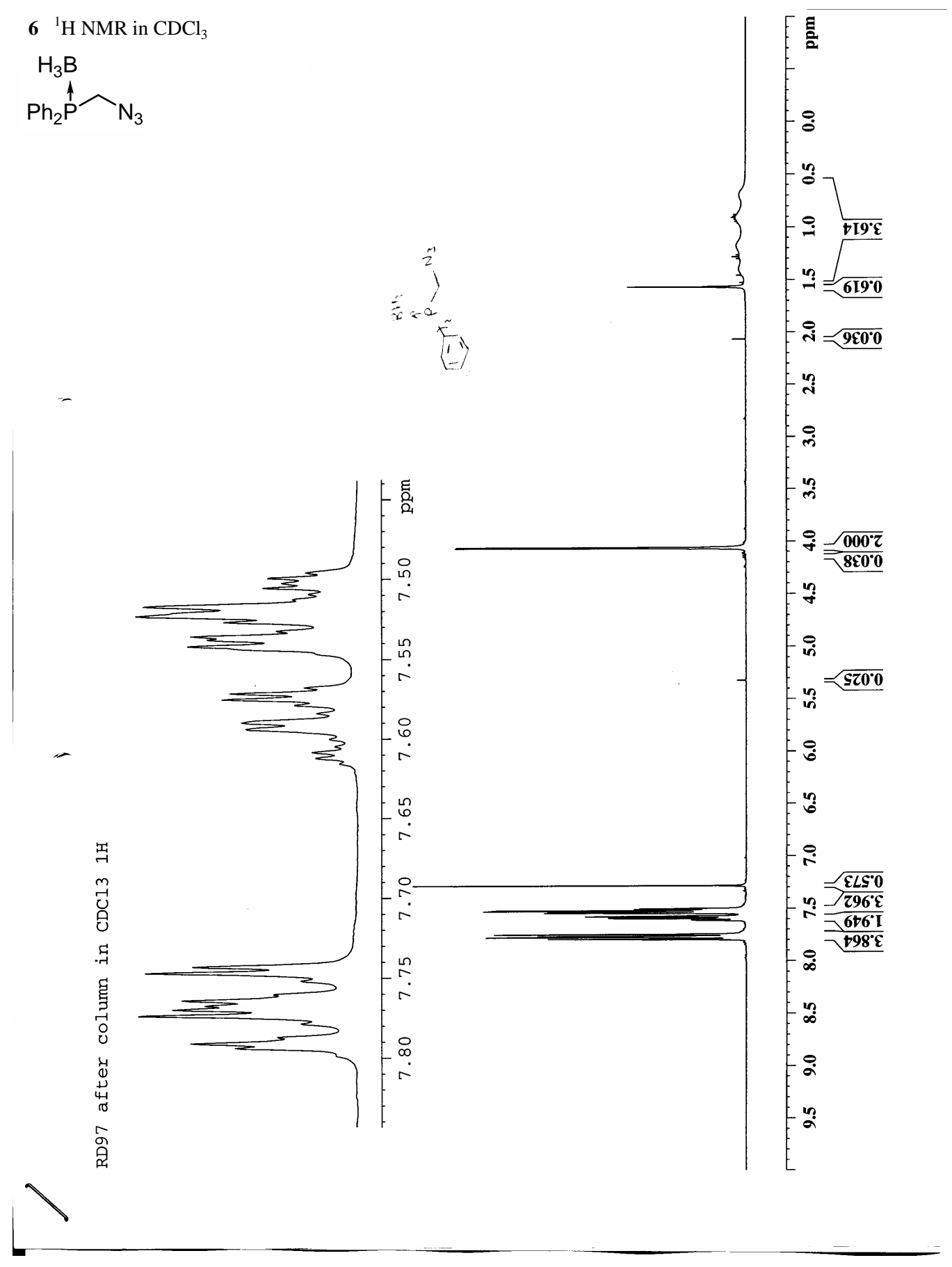


$6{ }^{13} \mathrm{C}$ NMR in $\mathrm{CDCl}_{3}$

- ${ }^{\mathrm{Hh}_{3} \mathrm{~B}} \stackrel{\stackrel{\mathrm{P}}{\mathrm{P}}}{{ }^{-}} \mathrm{N}_{3}$
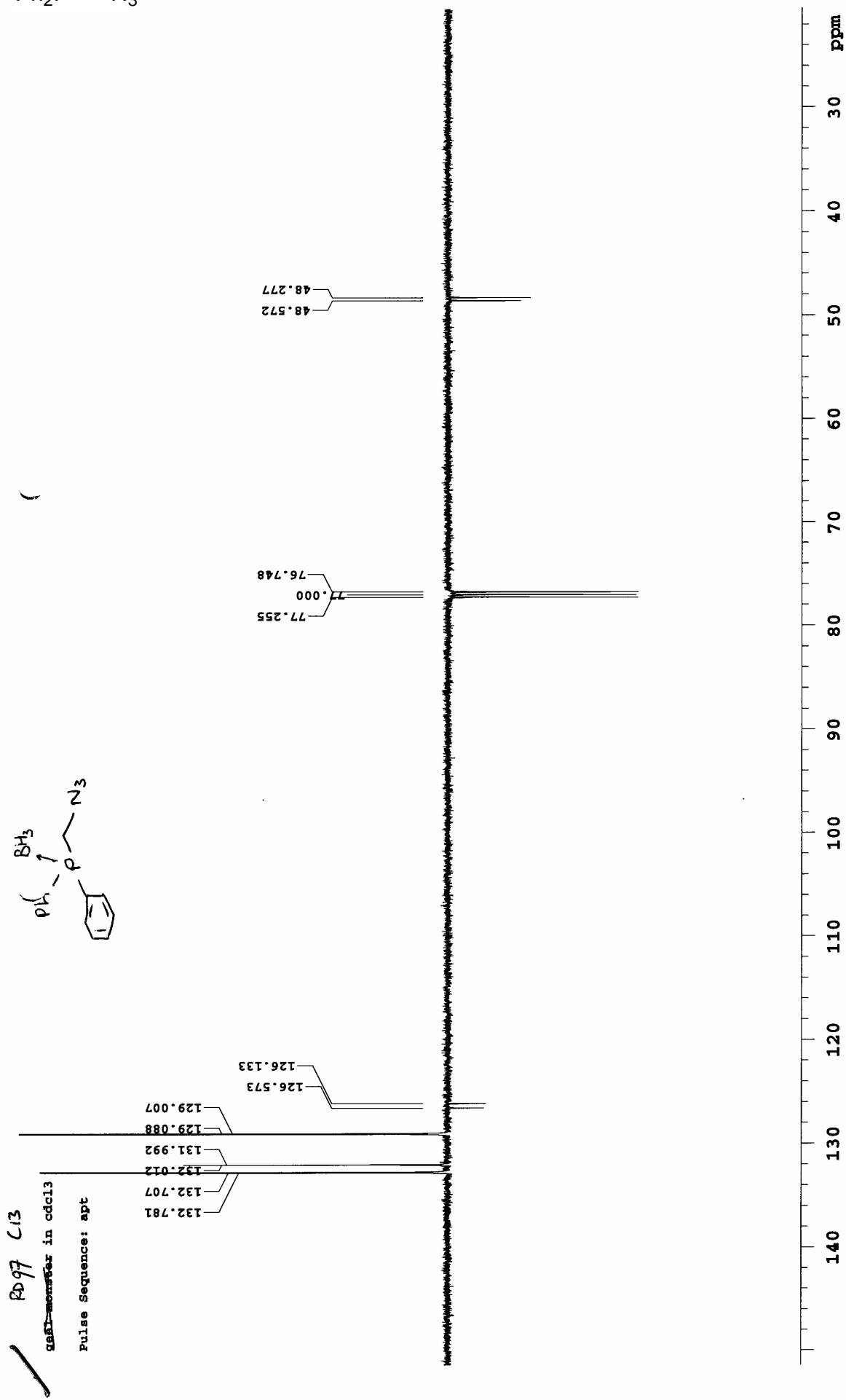


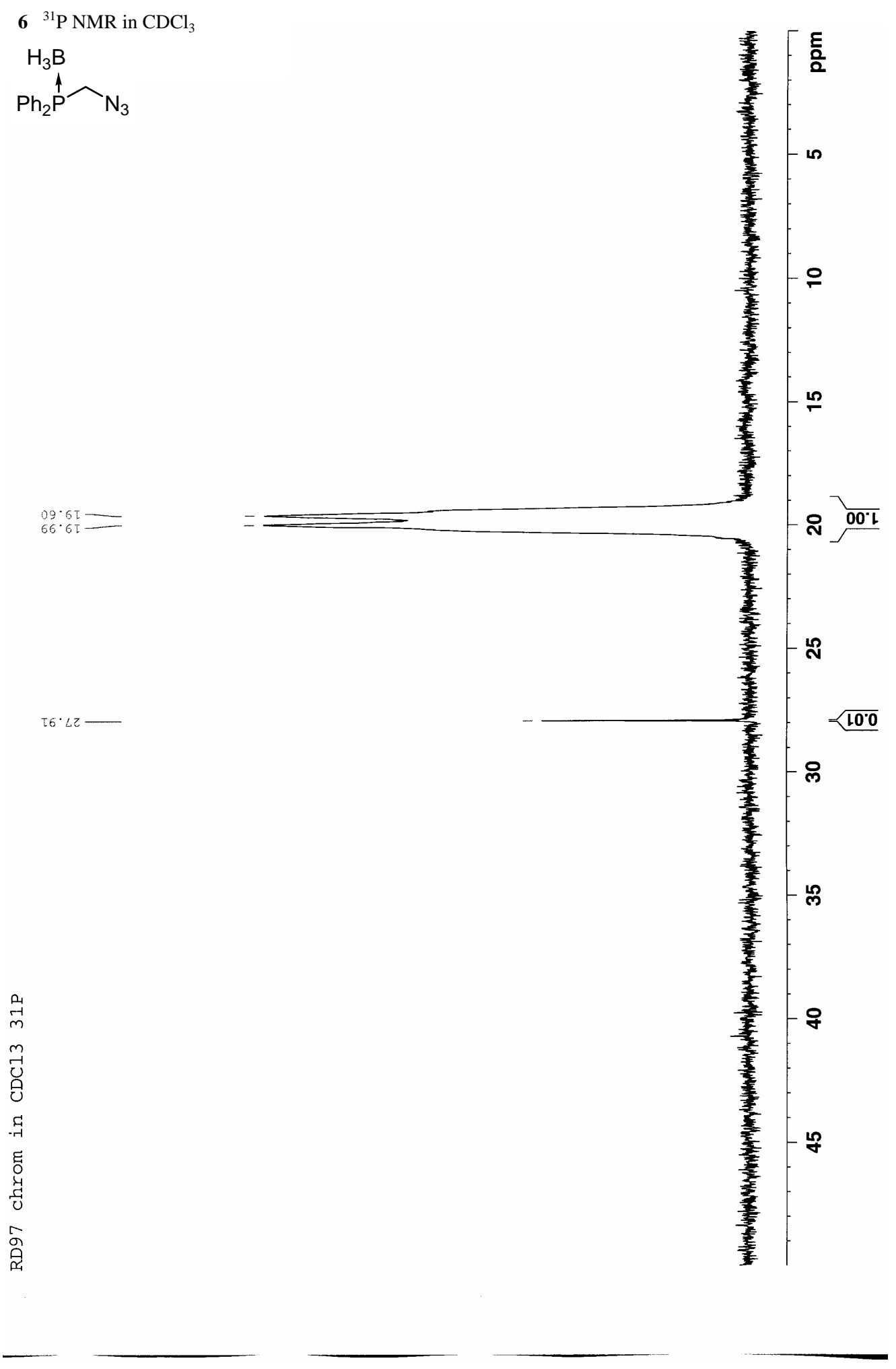


7a ${ }^{1} \mathrm{H}$ NMR in $\mathrm{CDCl}_{3}$

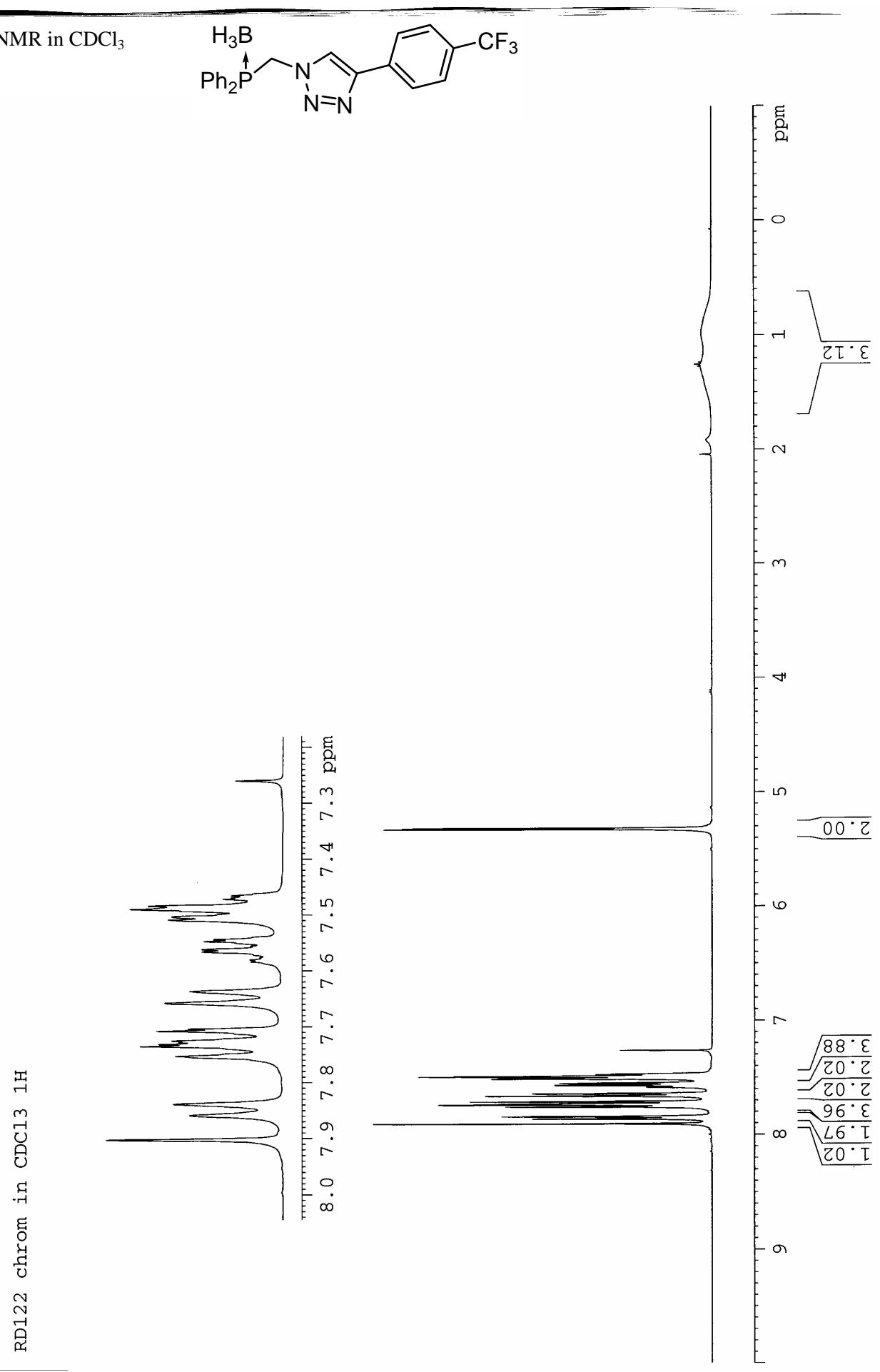


7a ${ }^{13} \mathrm{C} \mathrm{NMR} \mathrm{in} \mathrm{CDCl}_{3}$

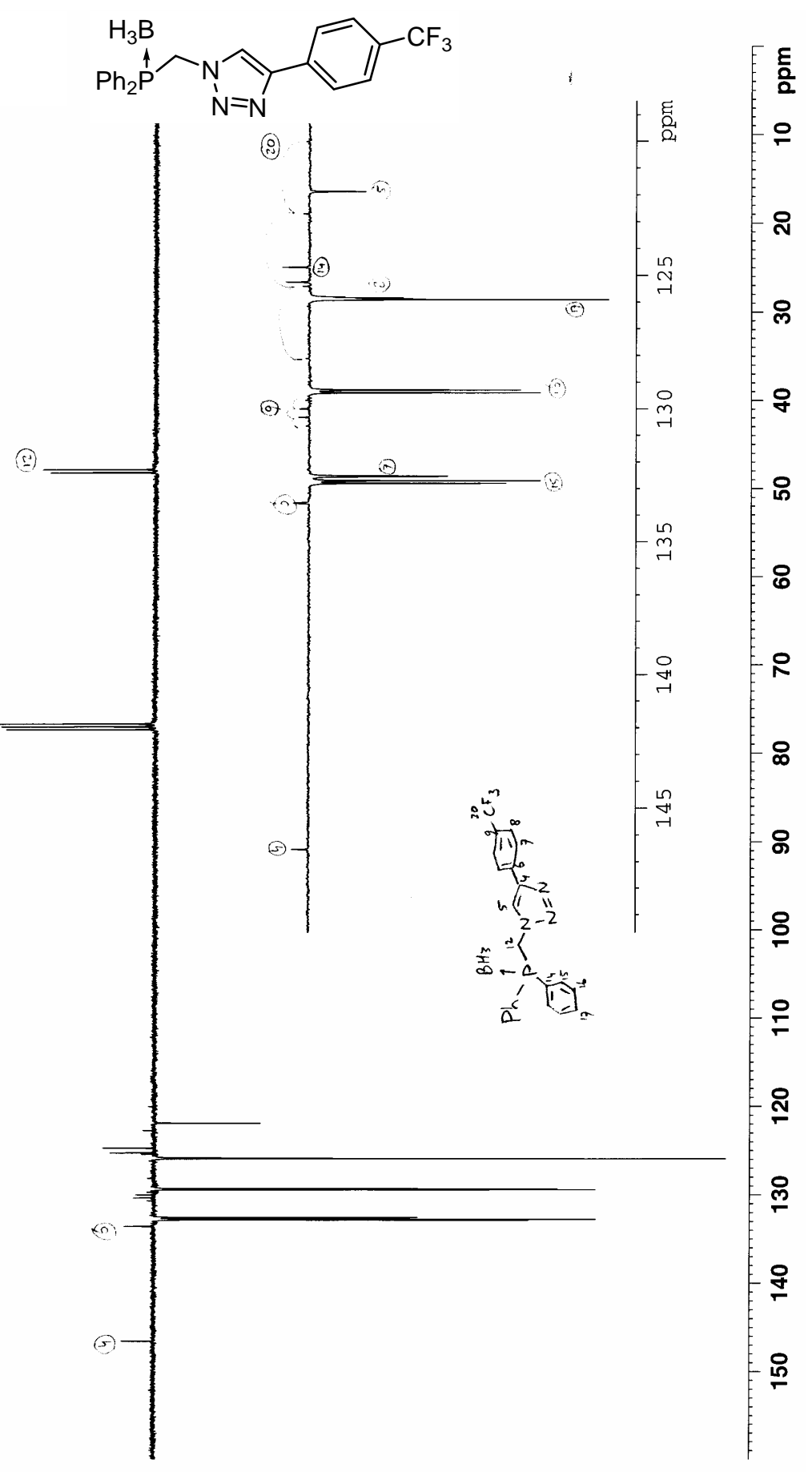




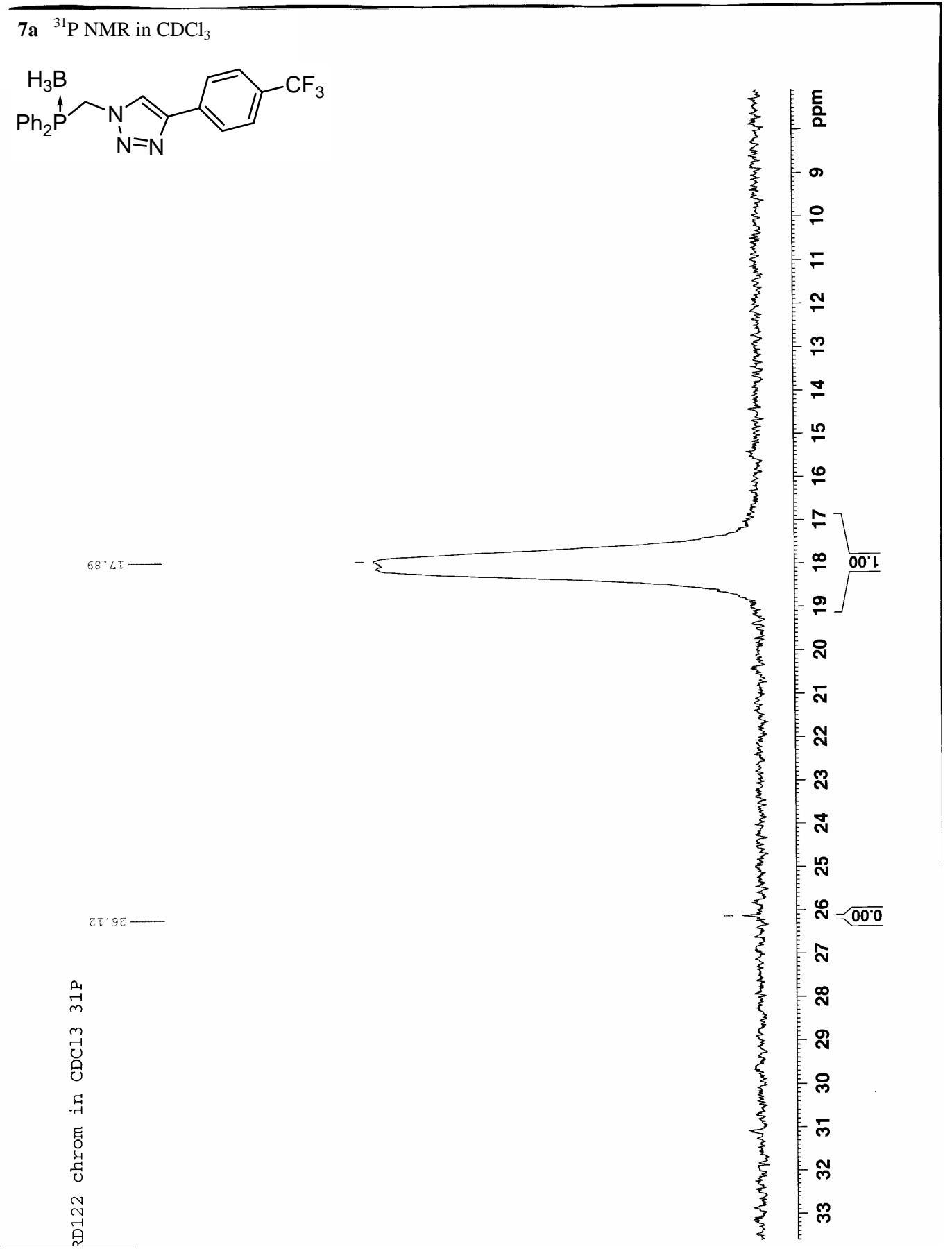




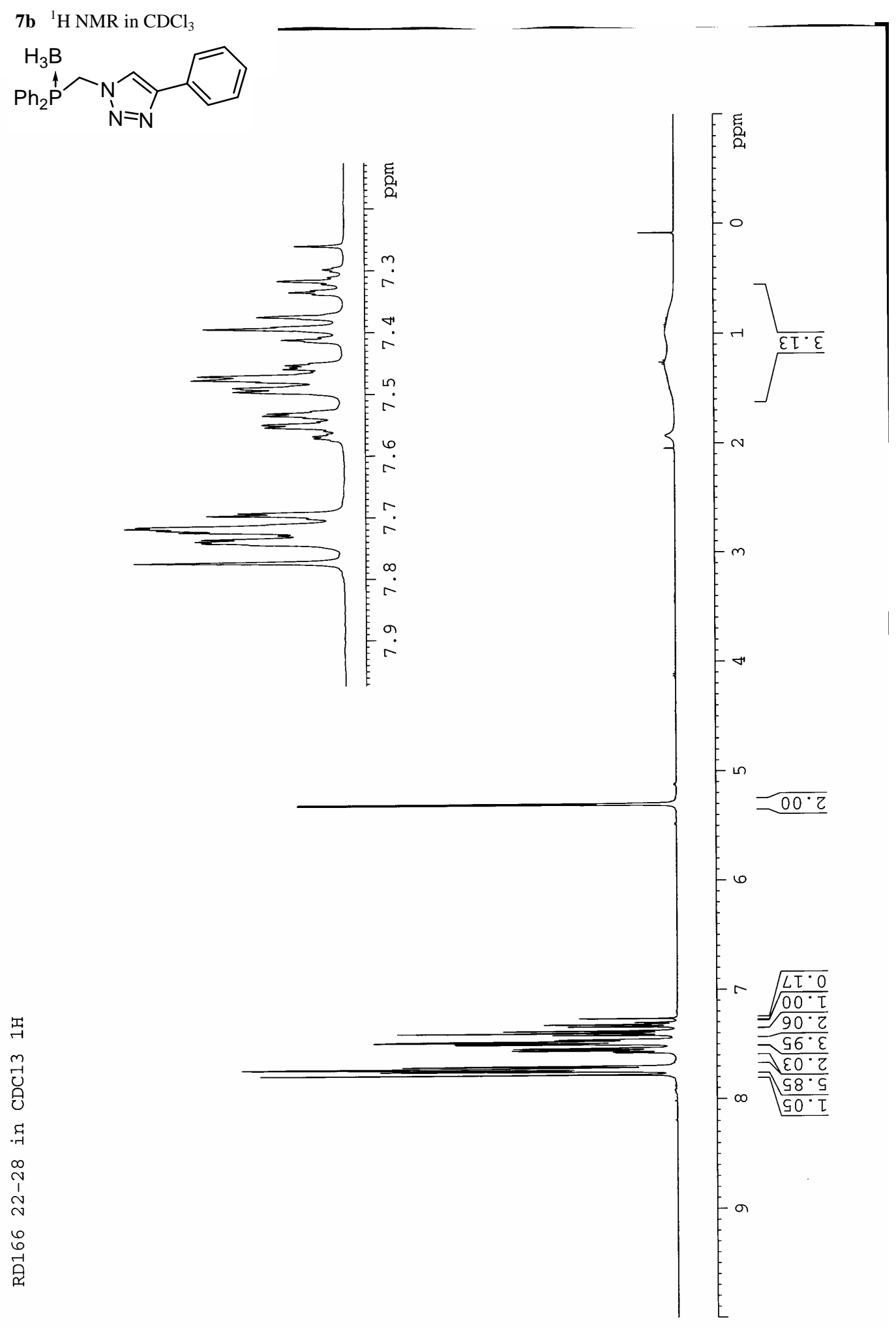




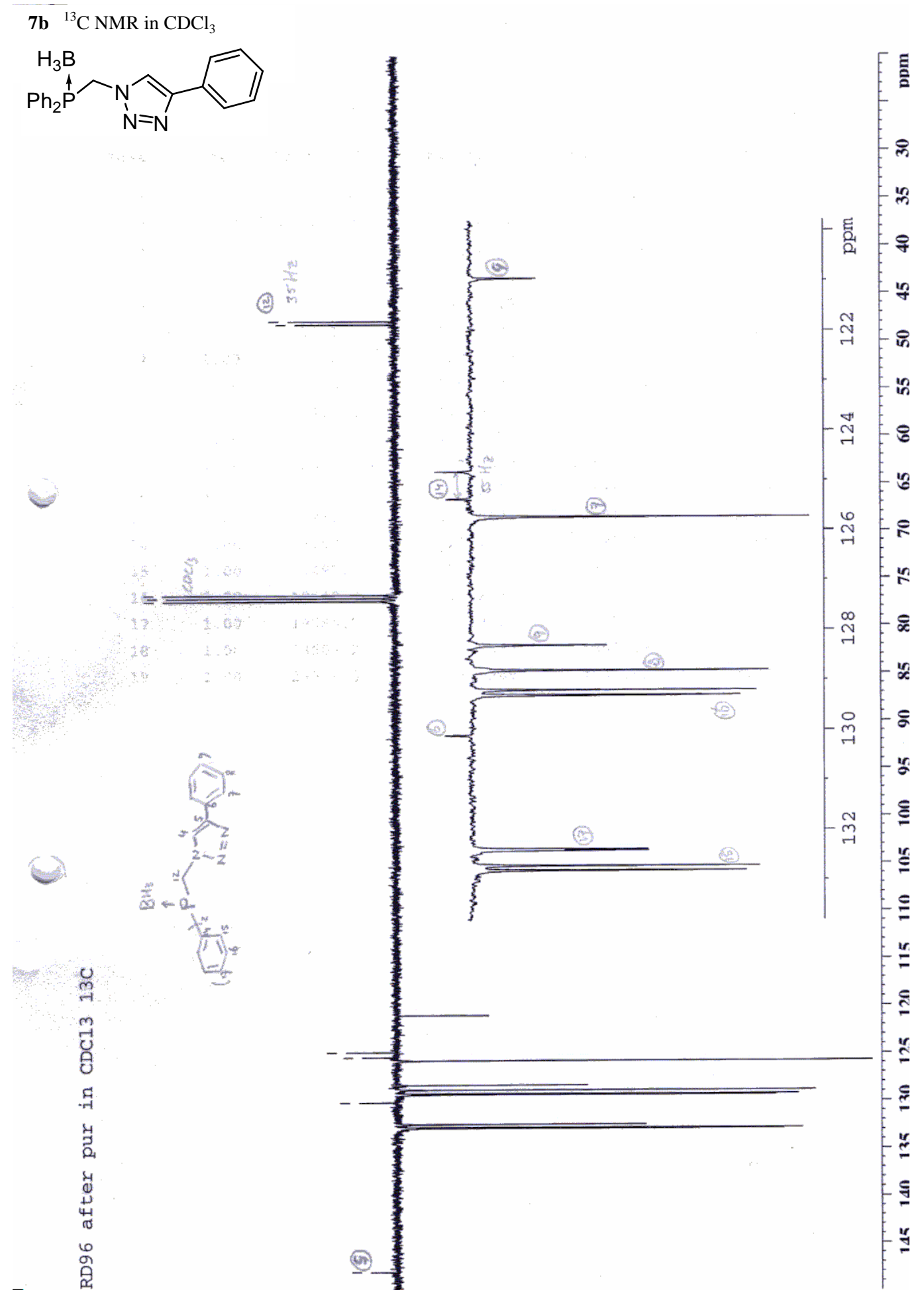




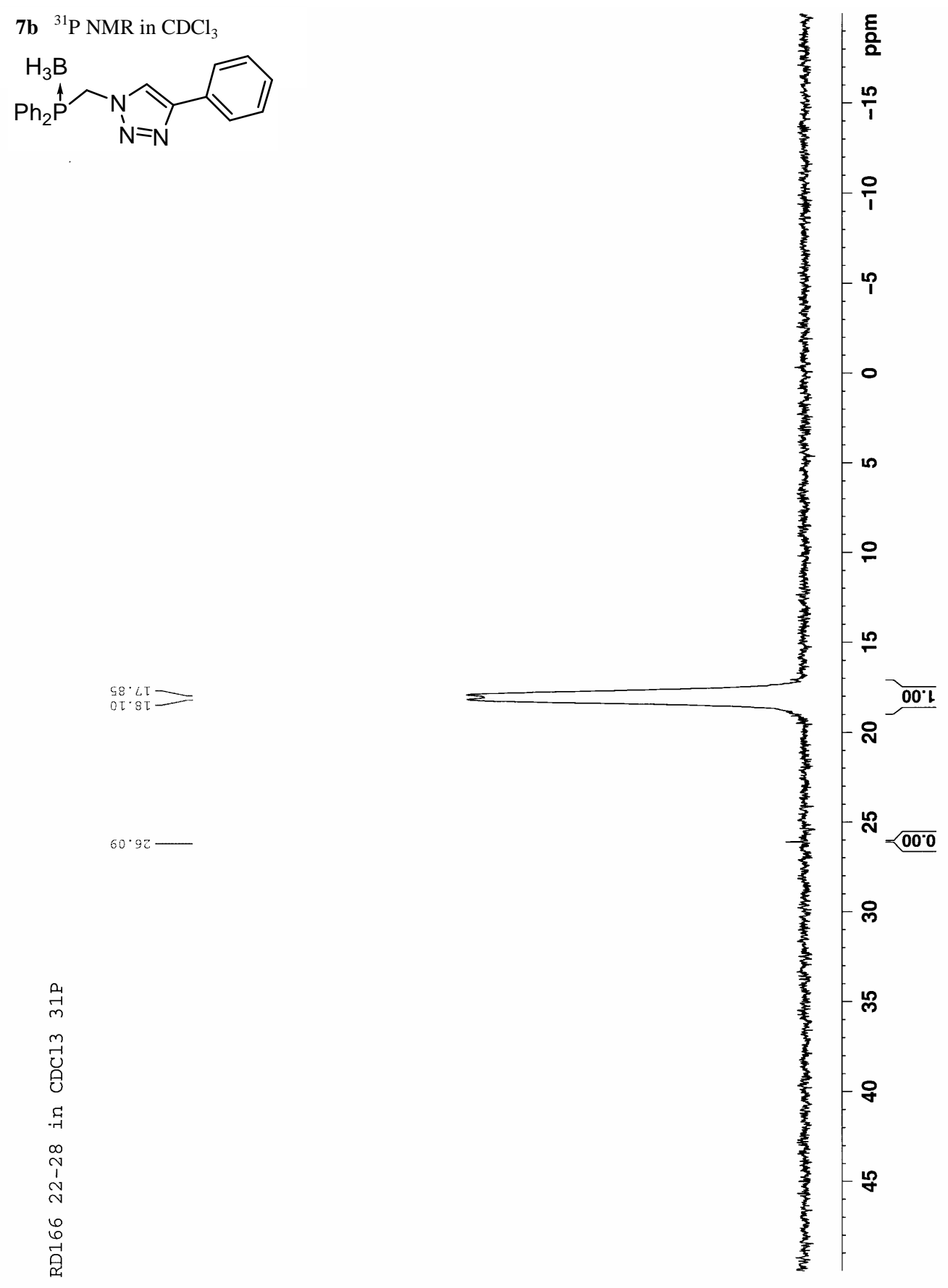




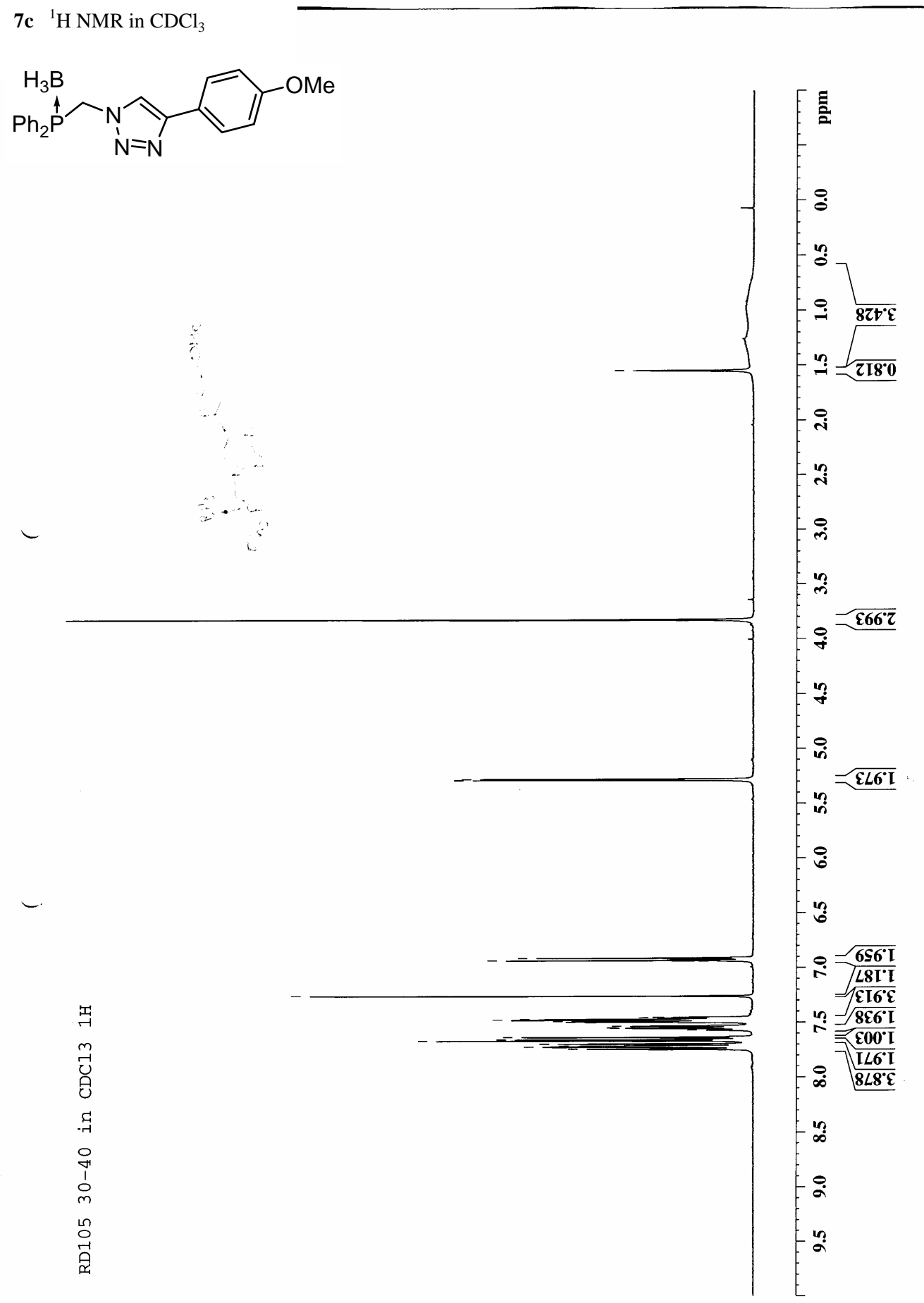




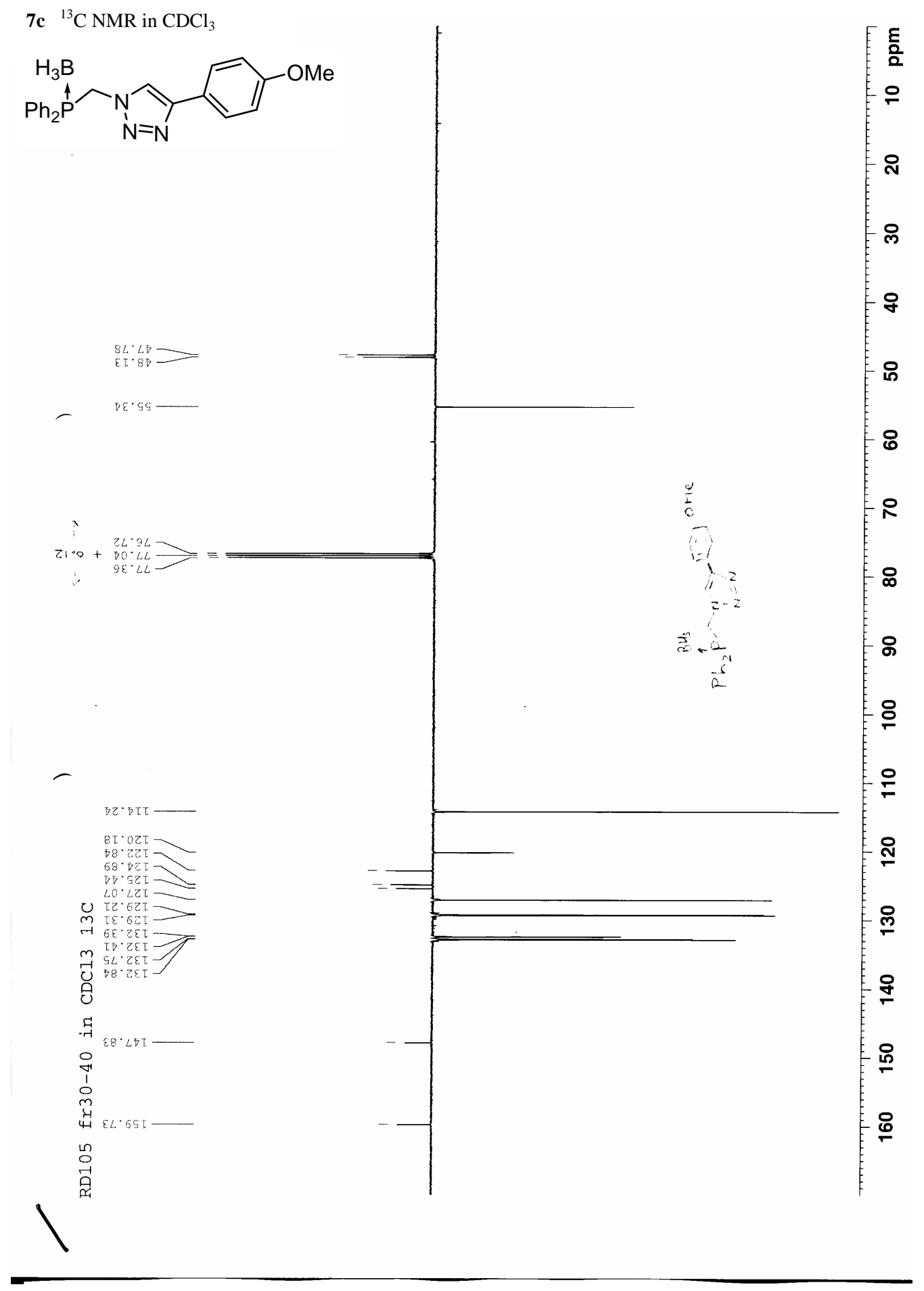




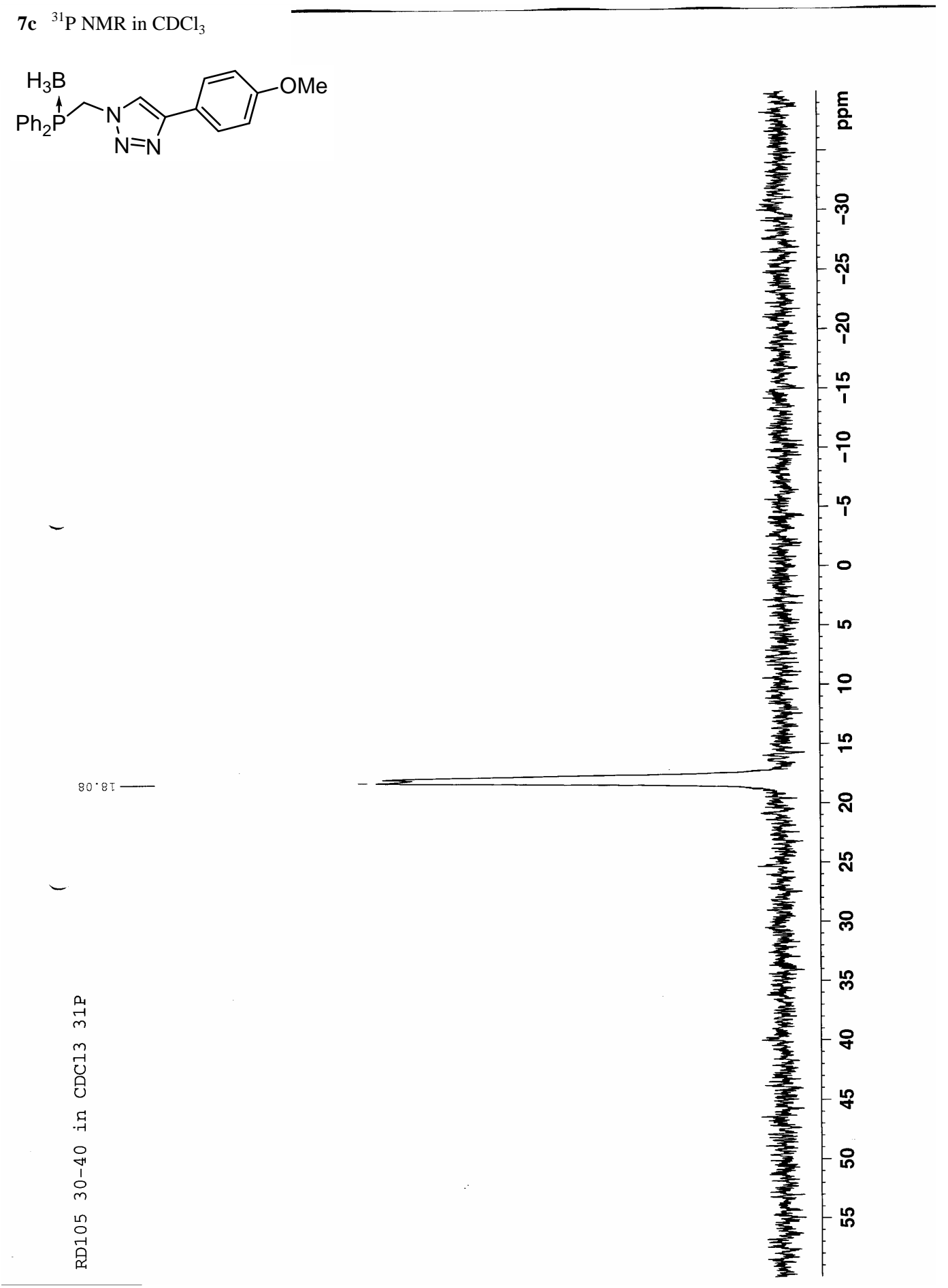


8a ${ }^{1} \mathrm{H} \mathrm{NMR}$ in $\mathrm{CDCl}_{3}$<smiles>FC(F)(F)c1ccc(-c2cn(CPc3ccccc3)nn2)cc1</smiles>

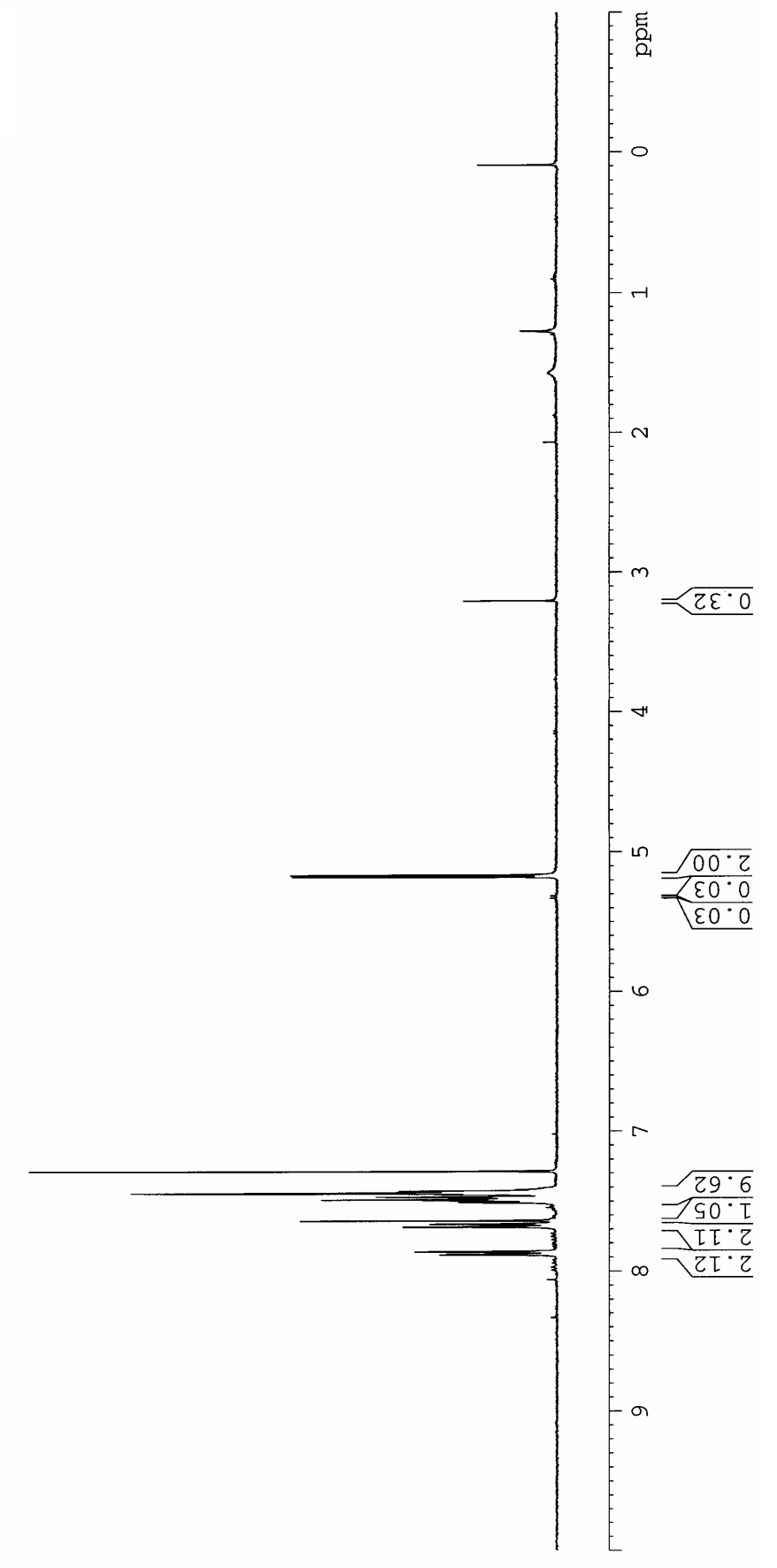

•

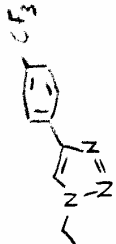

a

$\frac{\hat{a}}{a}$ 


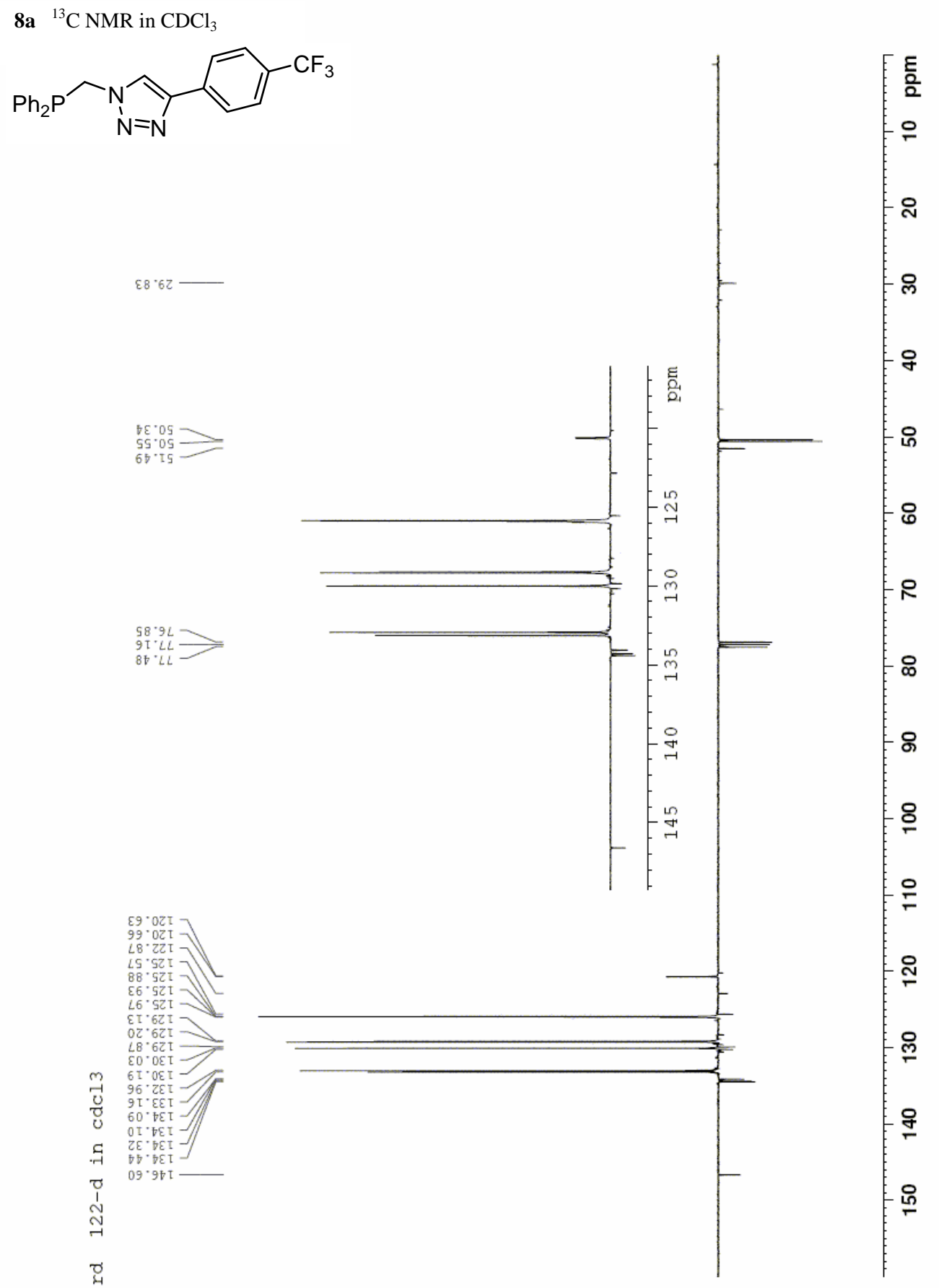


8a ${ }^{31} \mathrm{P}$ NMR in $\mathrm{CDCl}_{3}$

$\mathrm{Ph}_{2} \mathrm{P}$

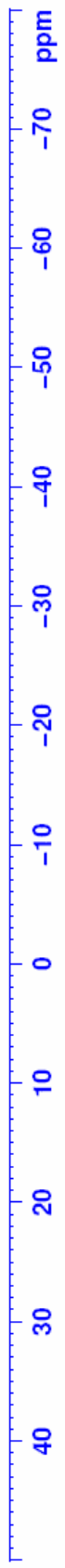


8b ${ }^{1} \mathrm{H} \mathrm{NMR}$ in $\mathrm{CDCl}_{3}$
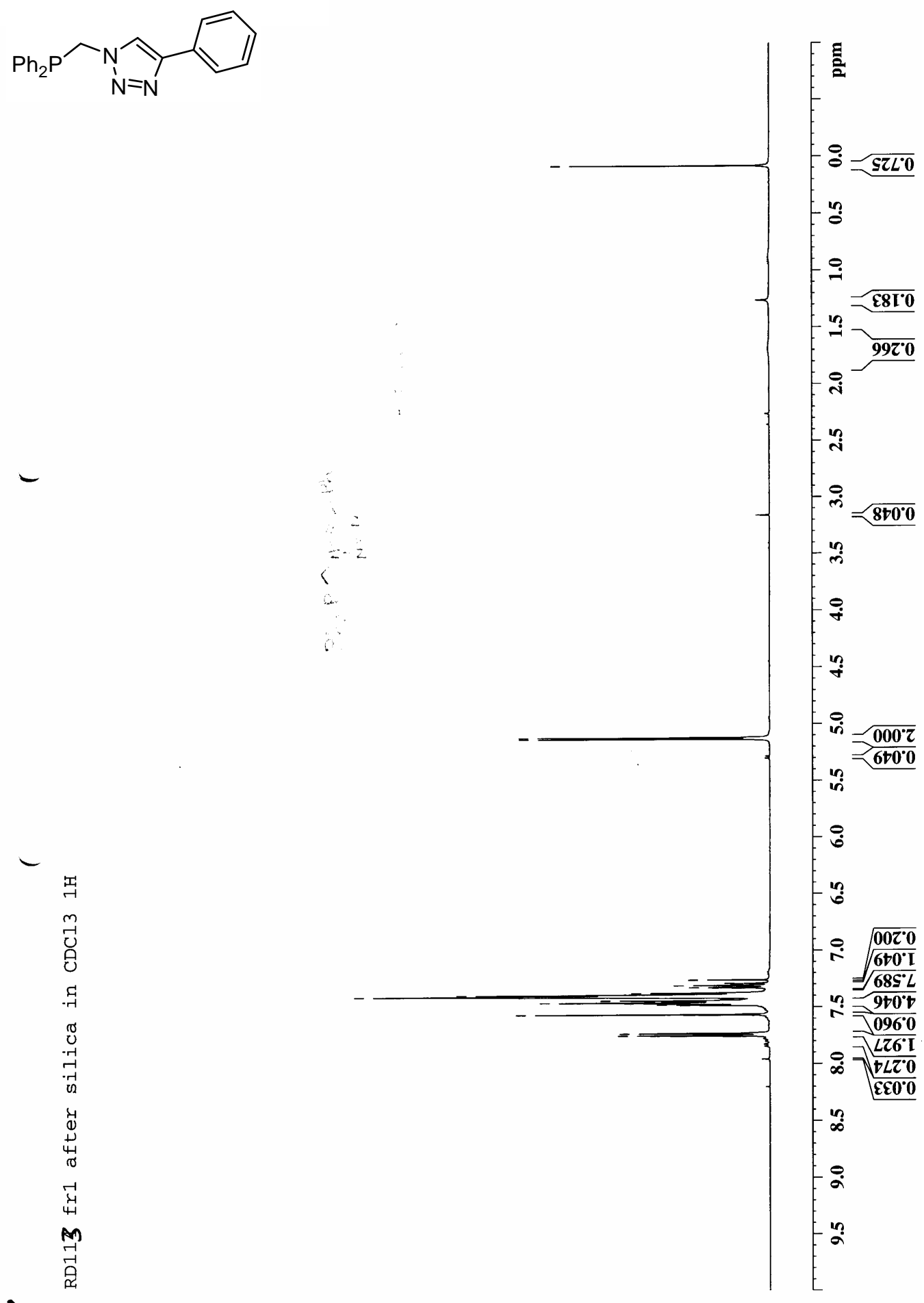

$Y$ 
8b ${ }^{13} \mathrm{C} \mathrm{NMR}$ in $\mathrm{CDCl}_{3}$

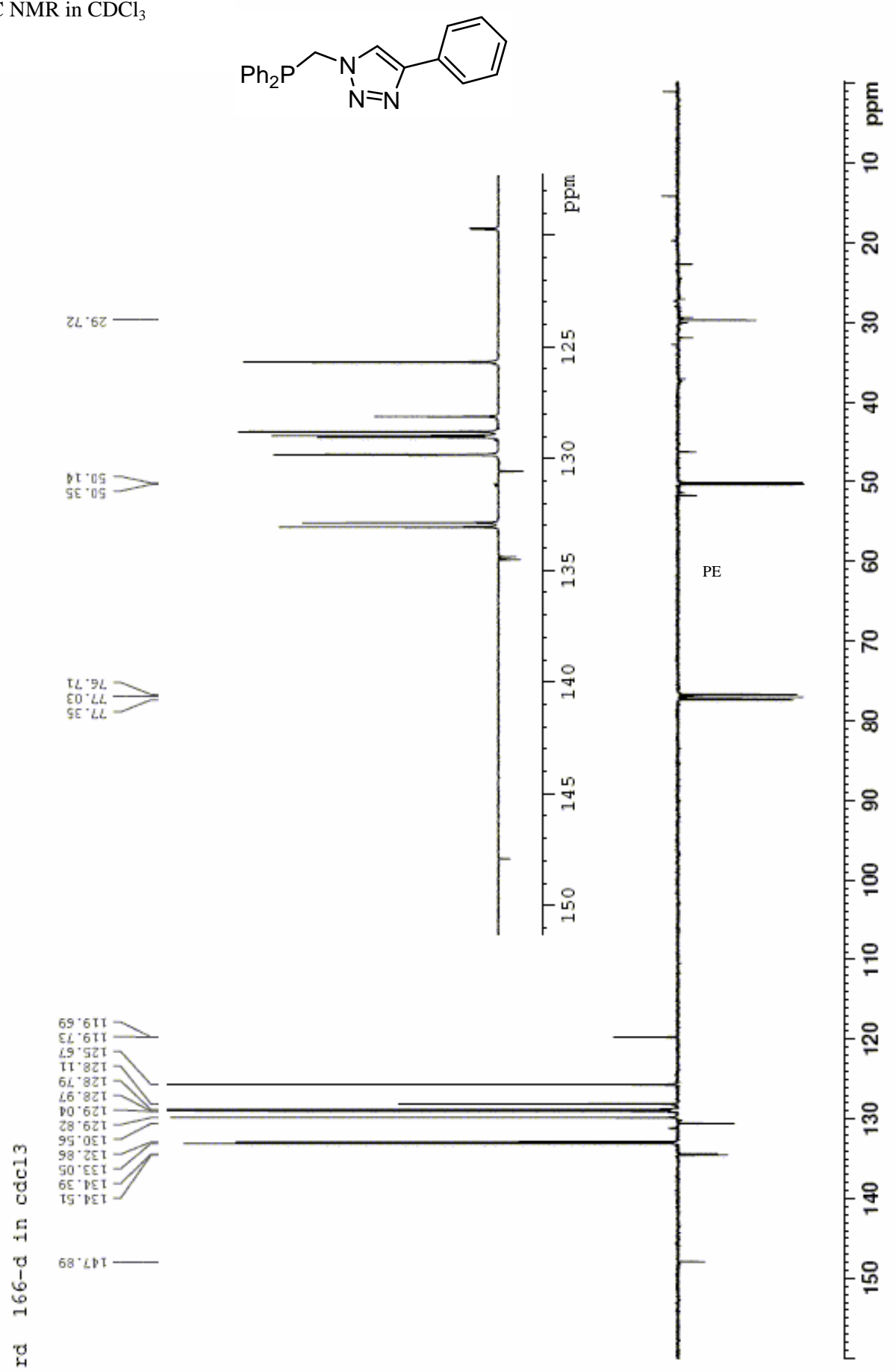




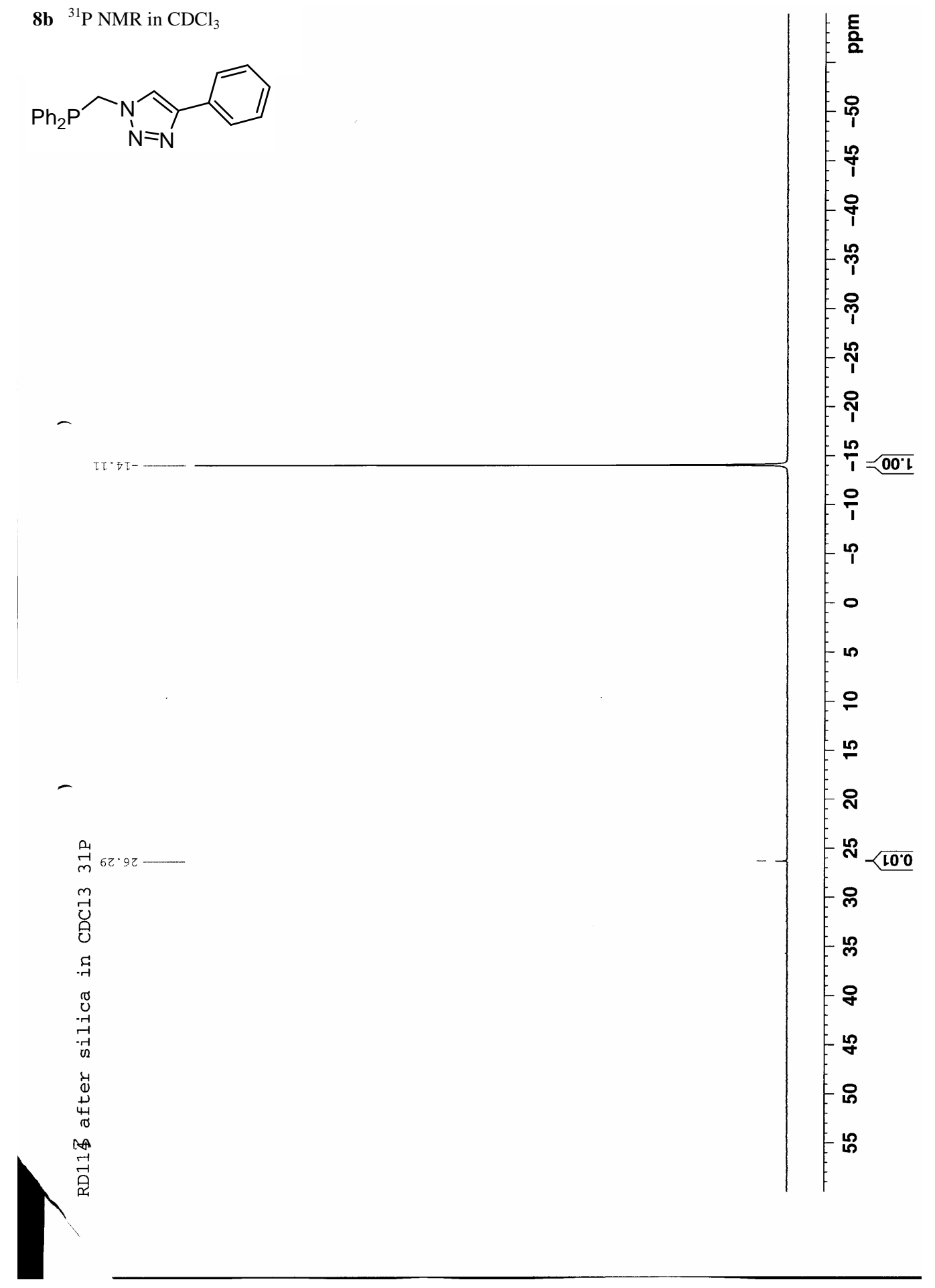


8c ${ }^{1} \mathrm{H}$ NMR in $\mathrm{CDCl}_{3}$

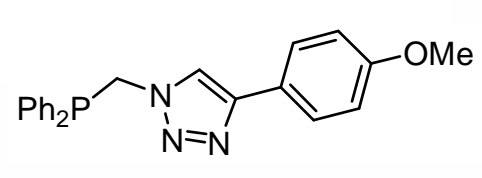

$\checkmark$

出

चे

.

苞

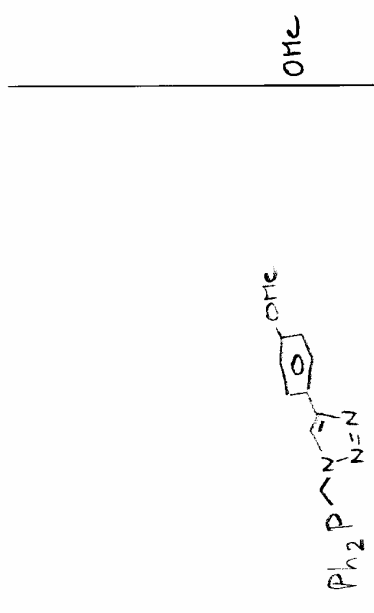

要 


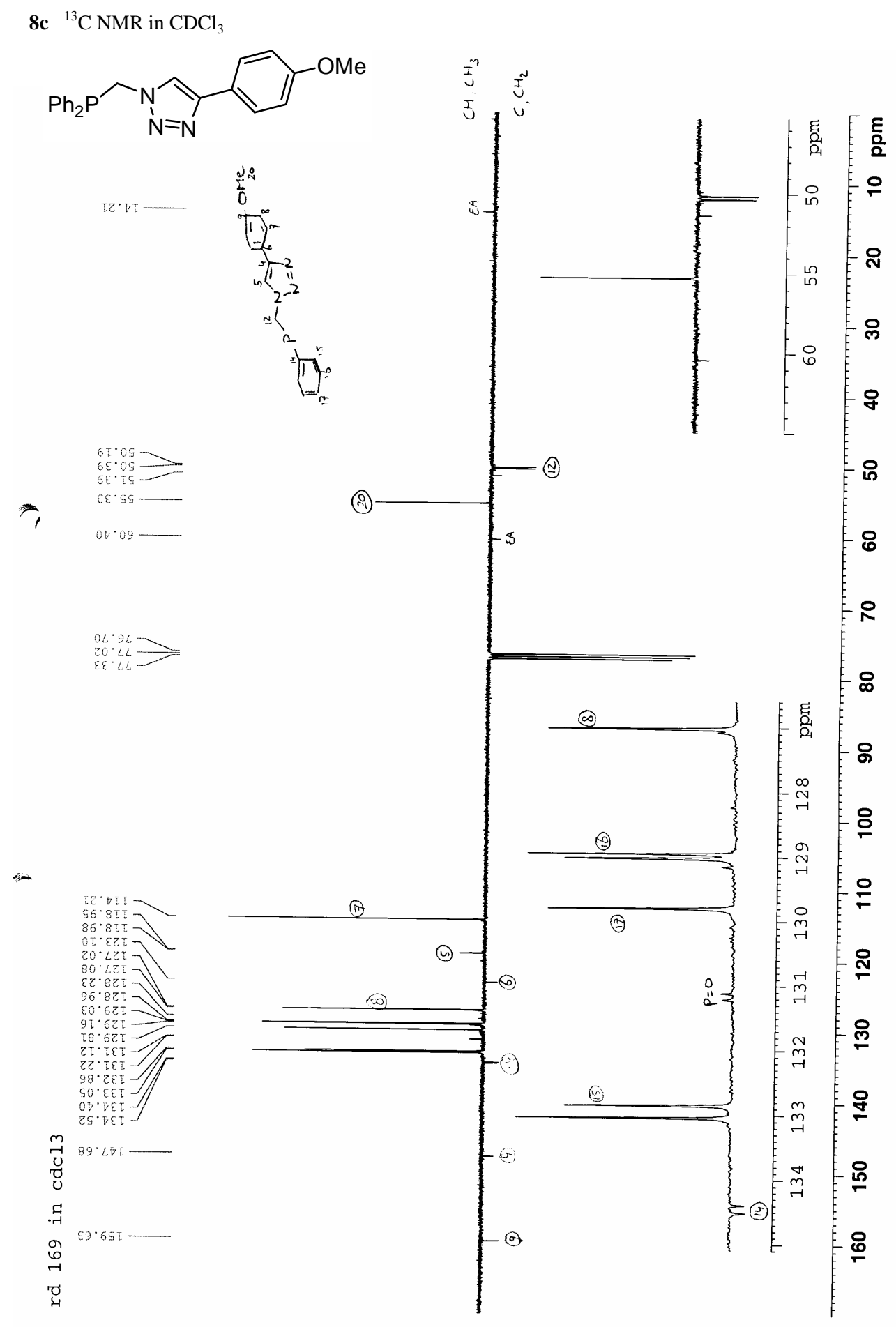




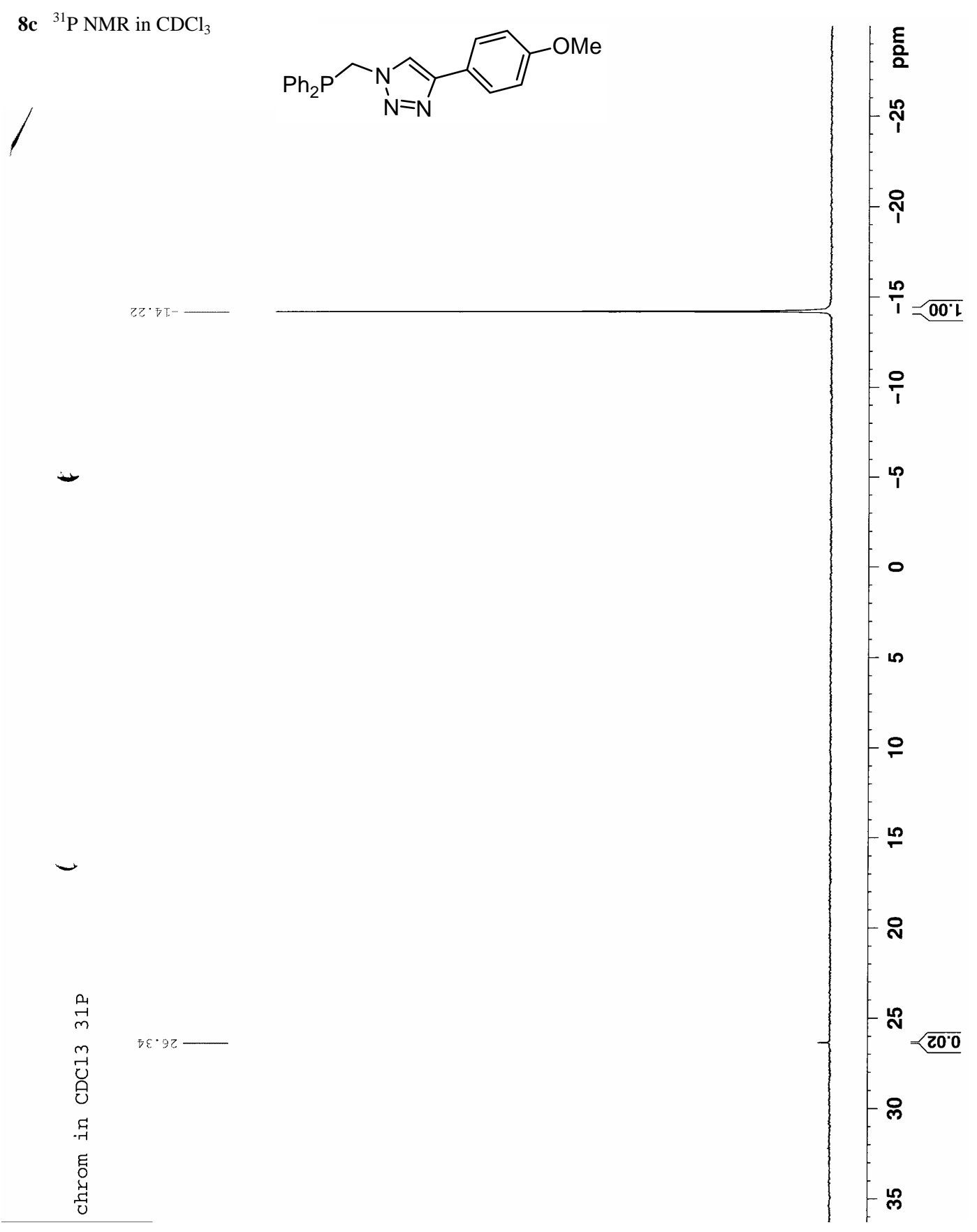




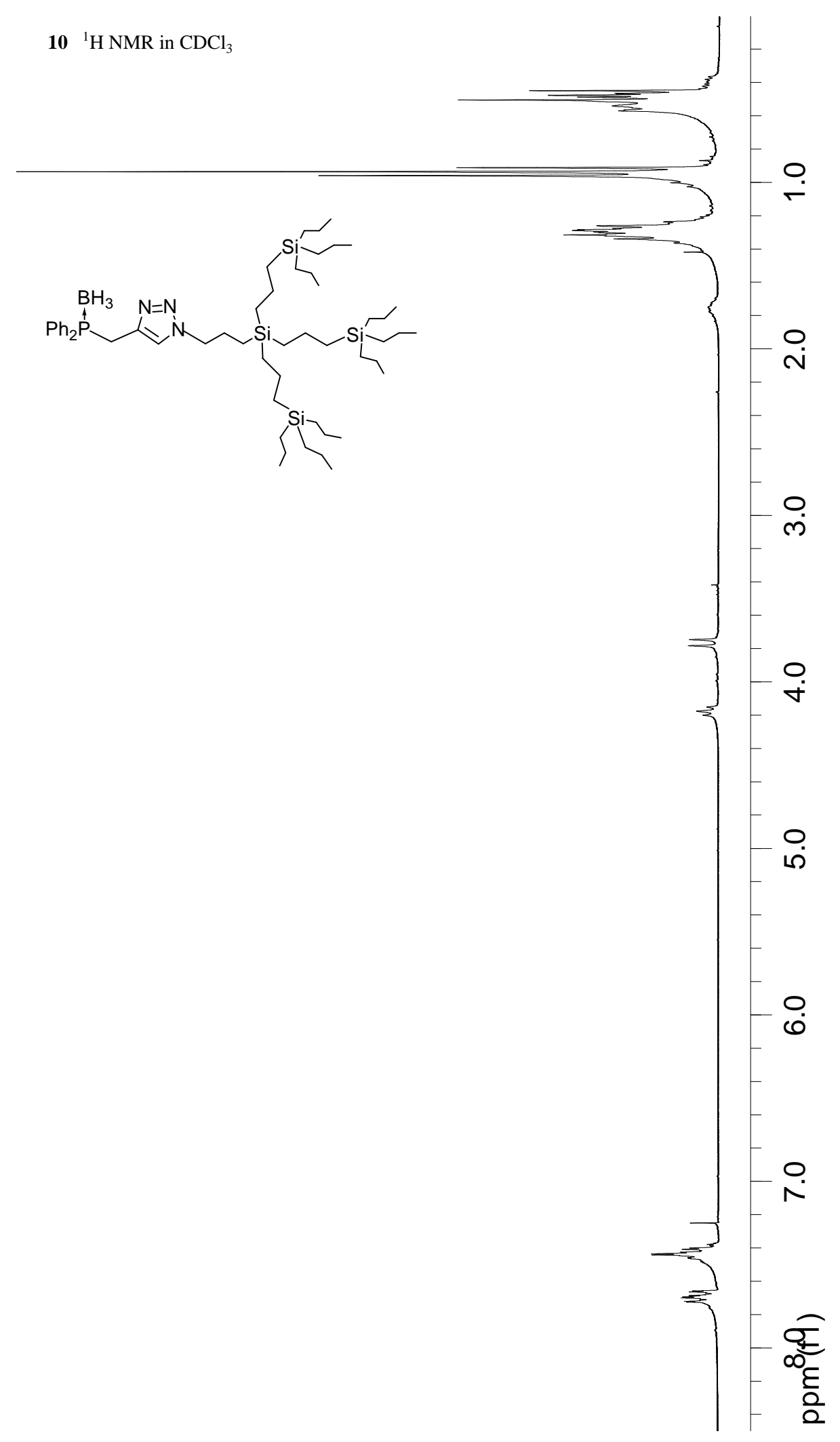




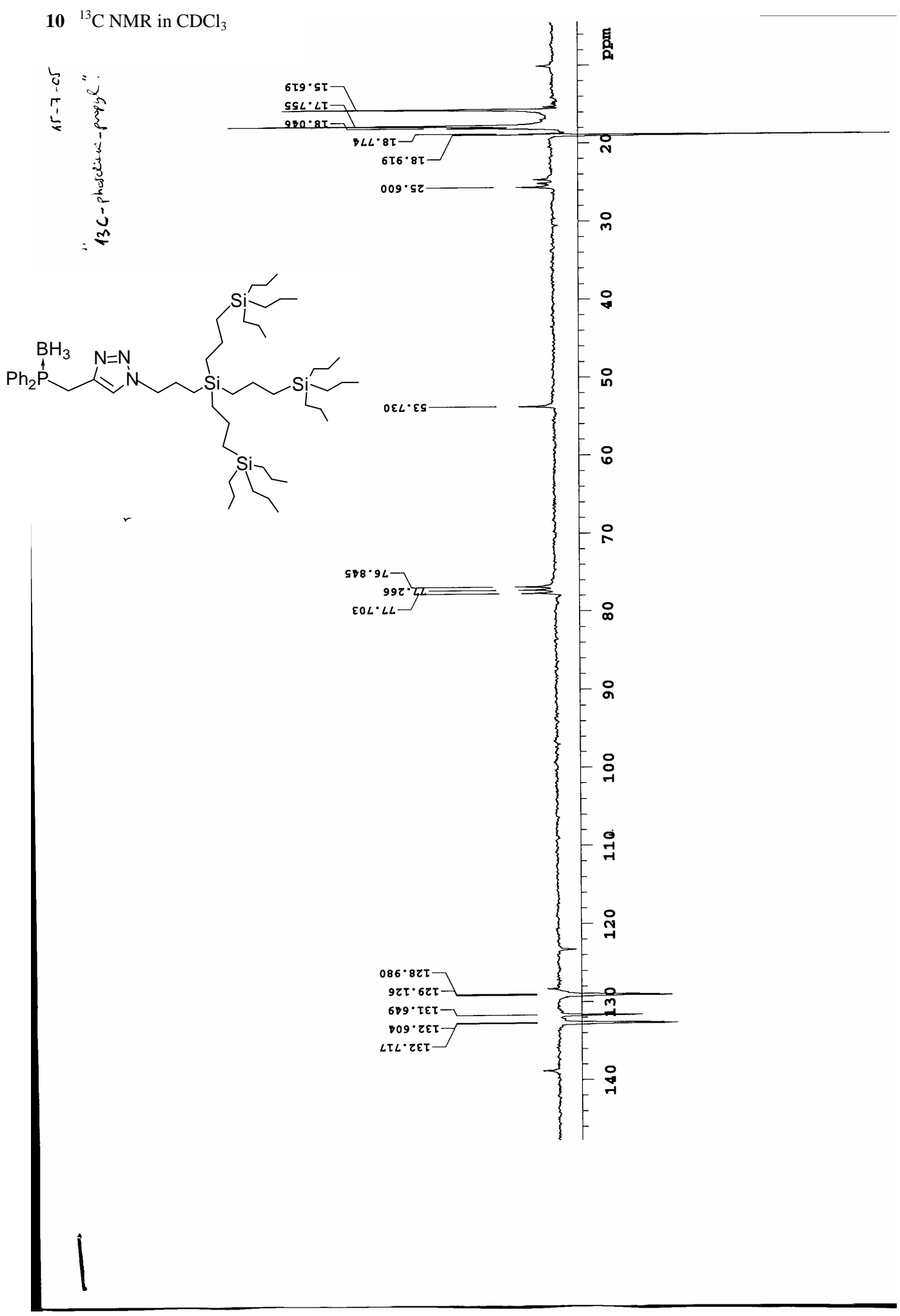




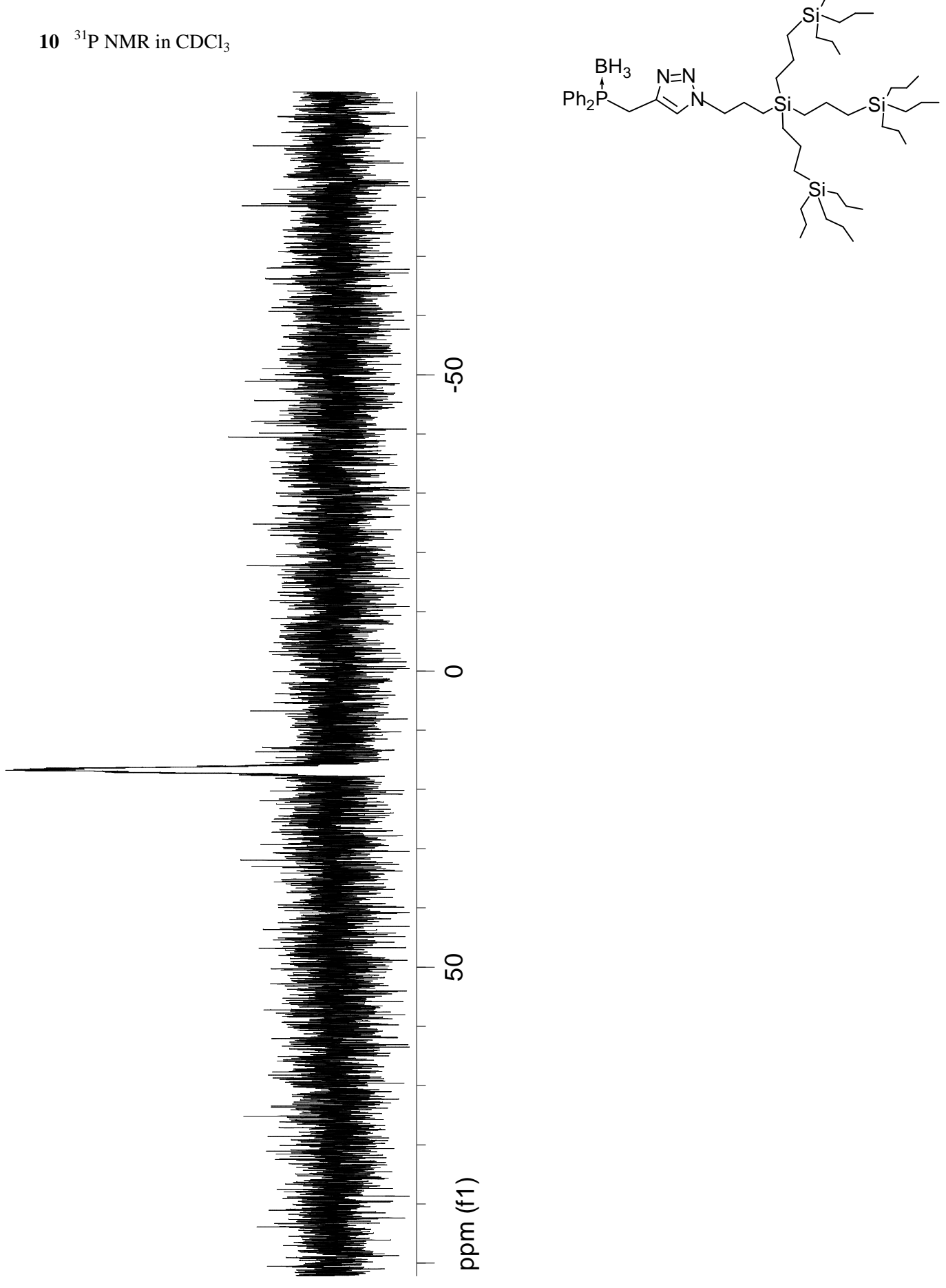




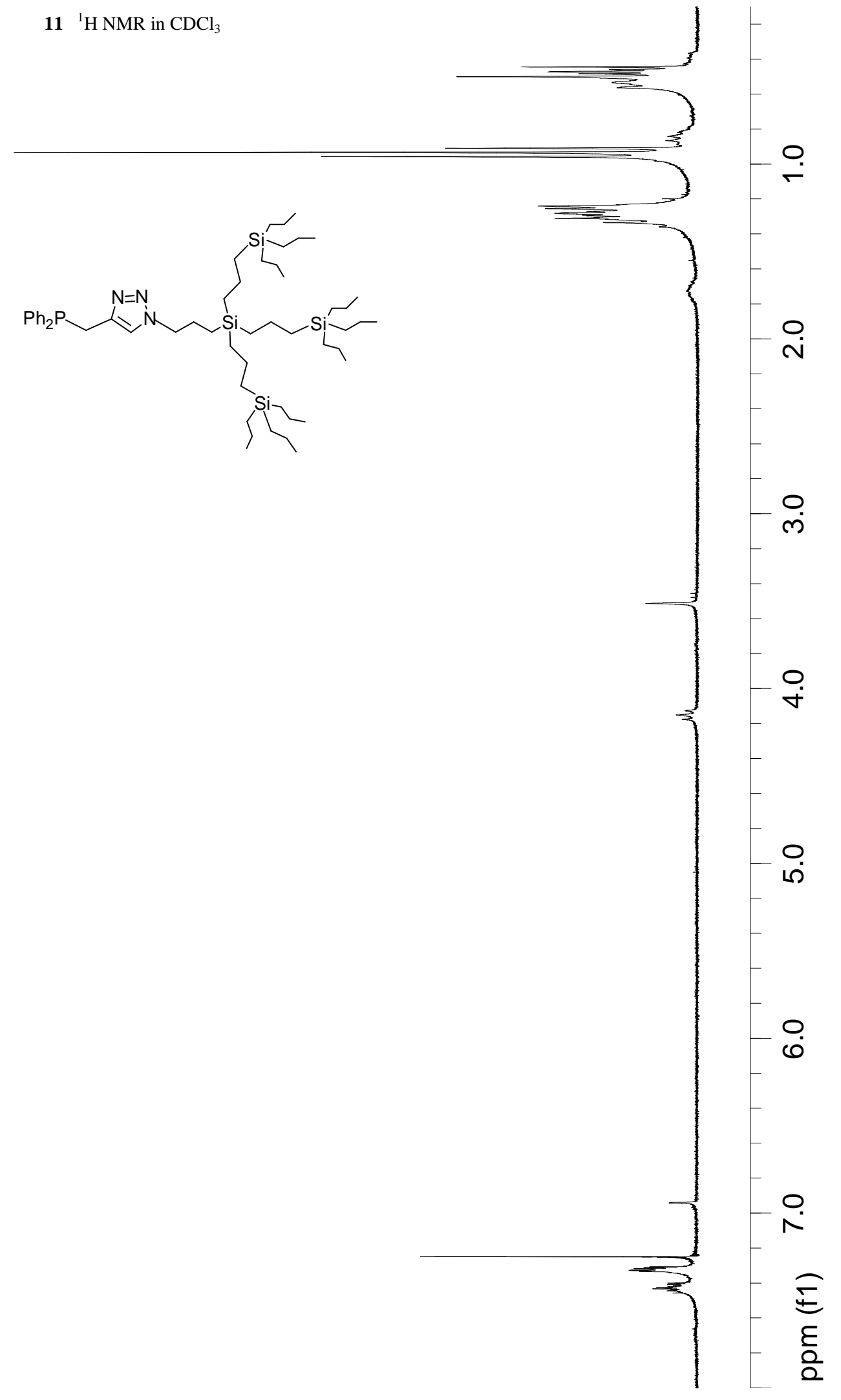


$11{ }^{13} \mathrm{C} \mathrm{NMR}$ in $\mathrm{CDCl}_{3}$

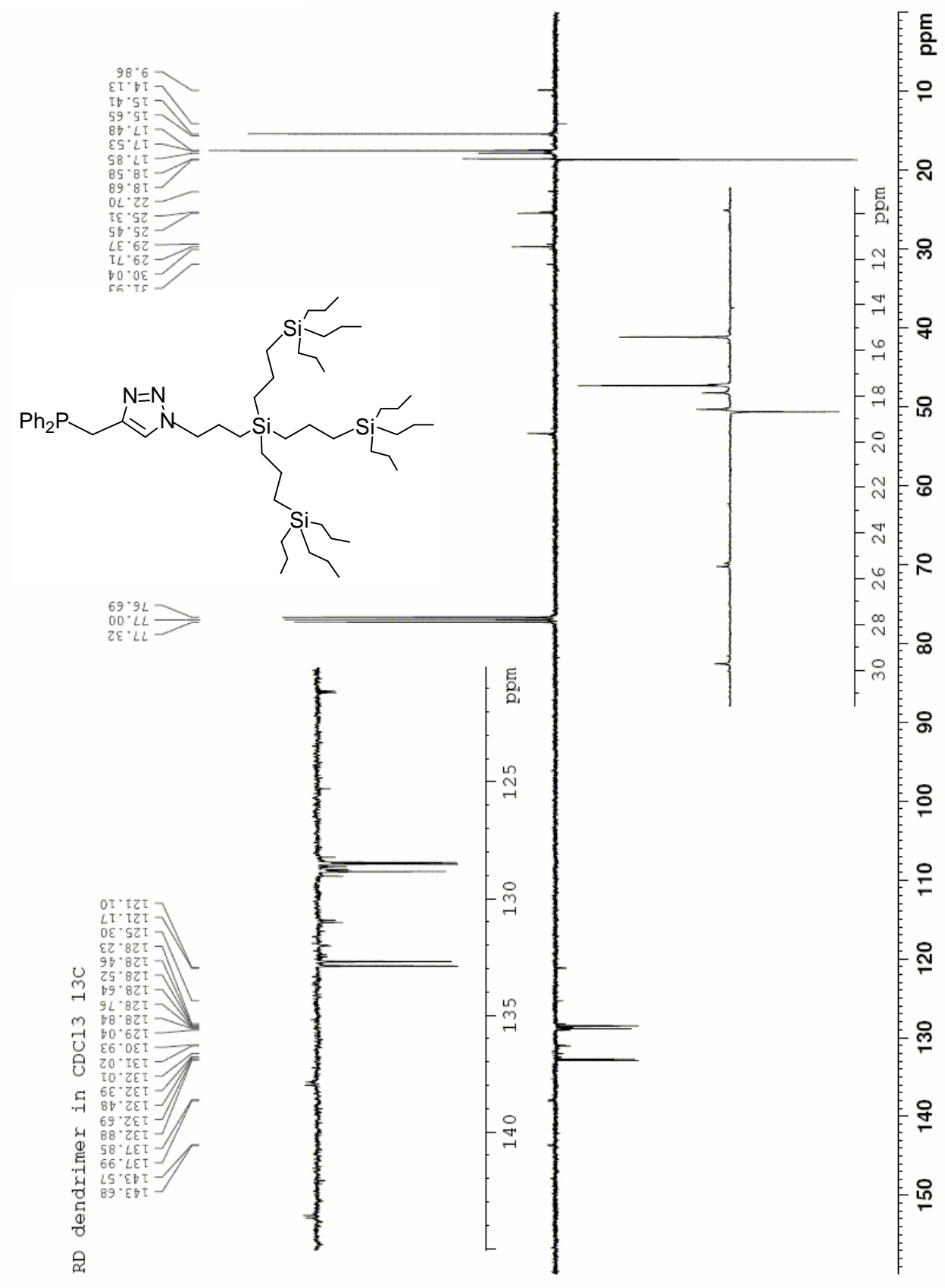









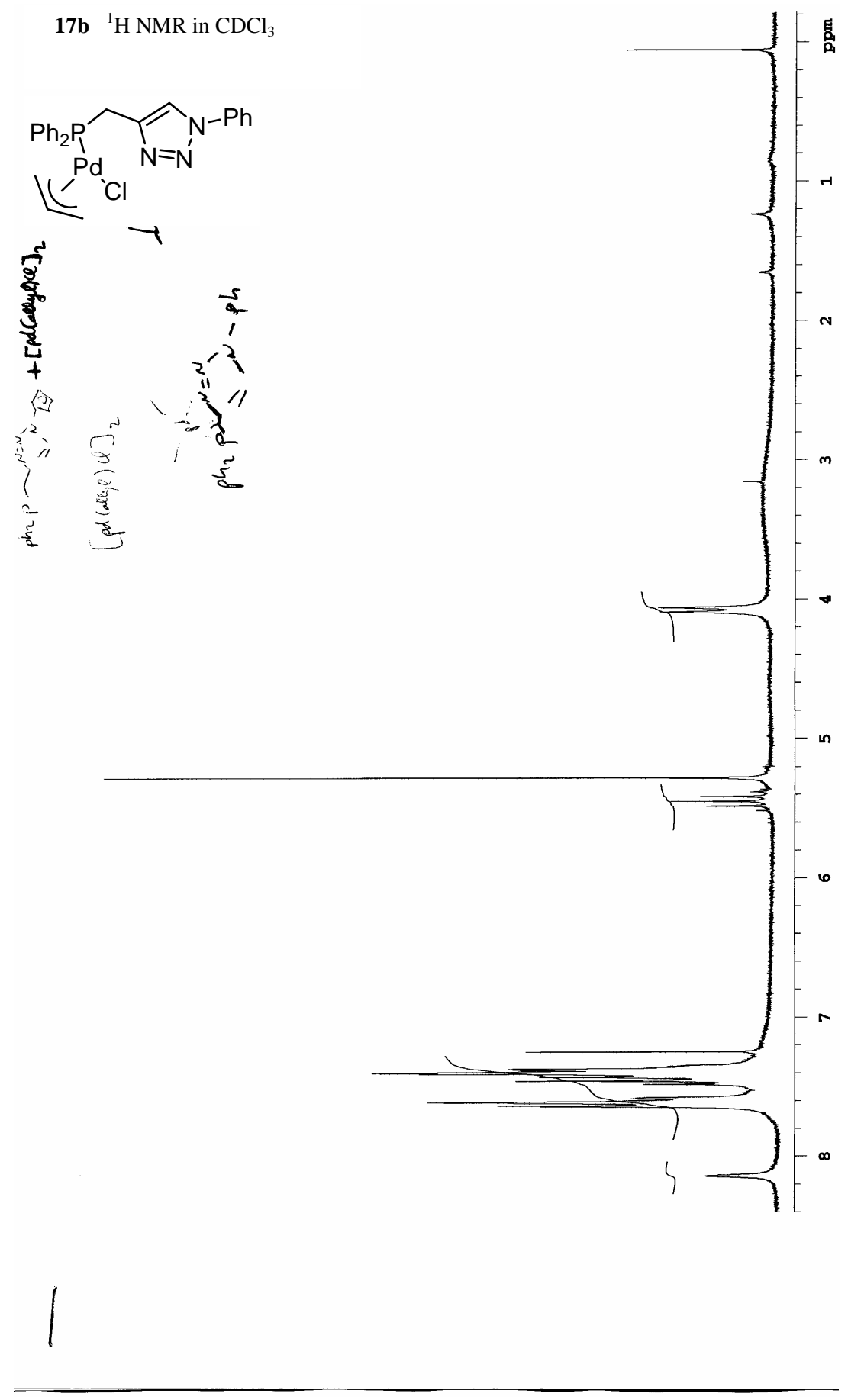




$$
\text { 17b }{ }^{31} \mathrm{P} \mathrm{NMR} \text { in } \mathrm{CDCl}_{3}
$$
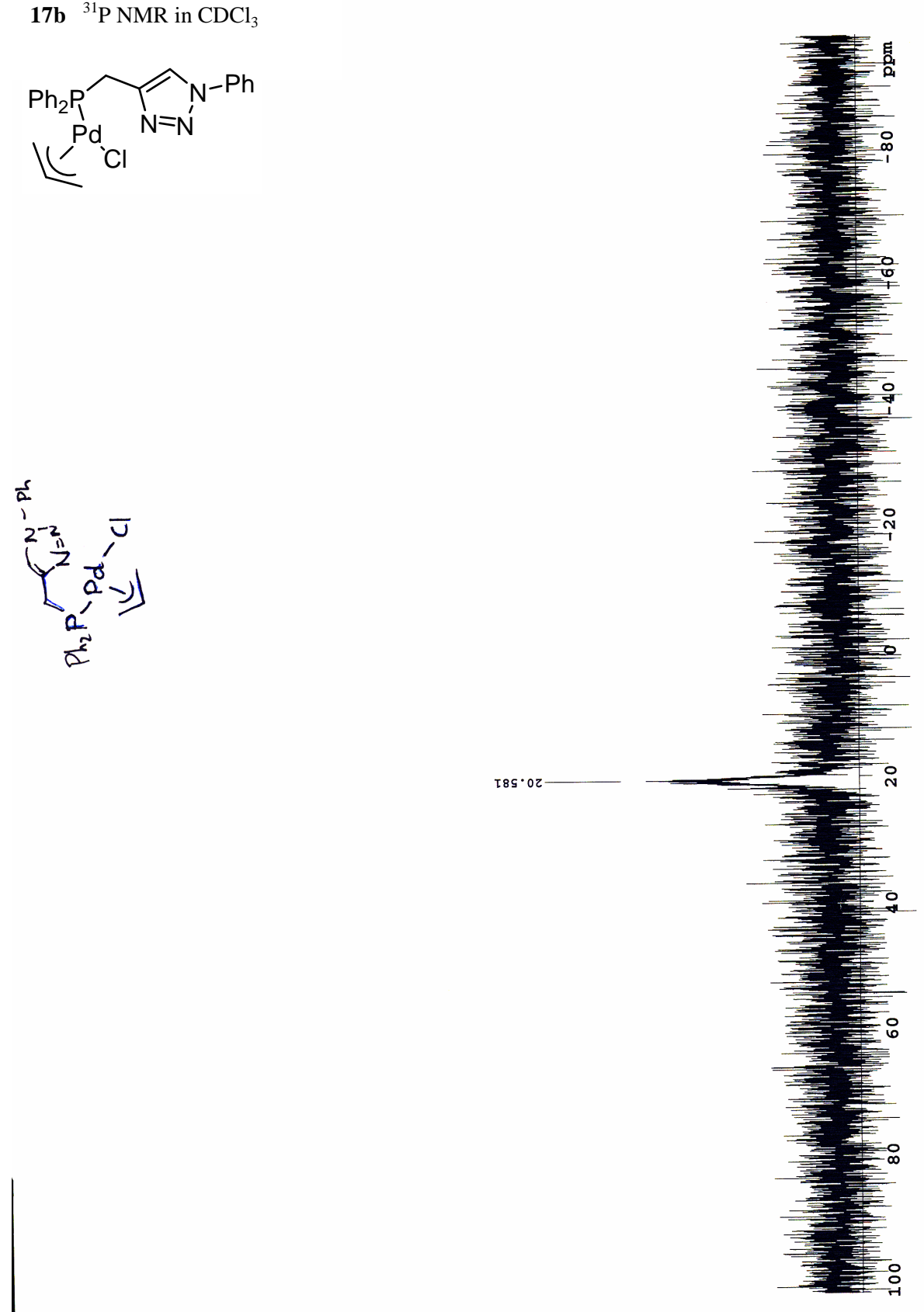


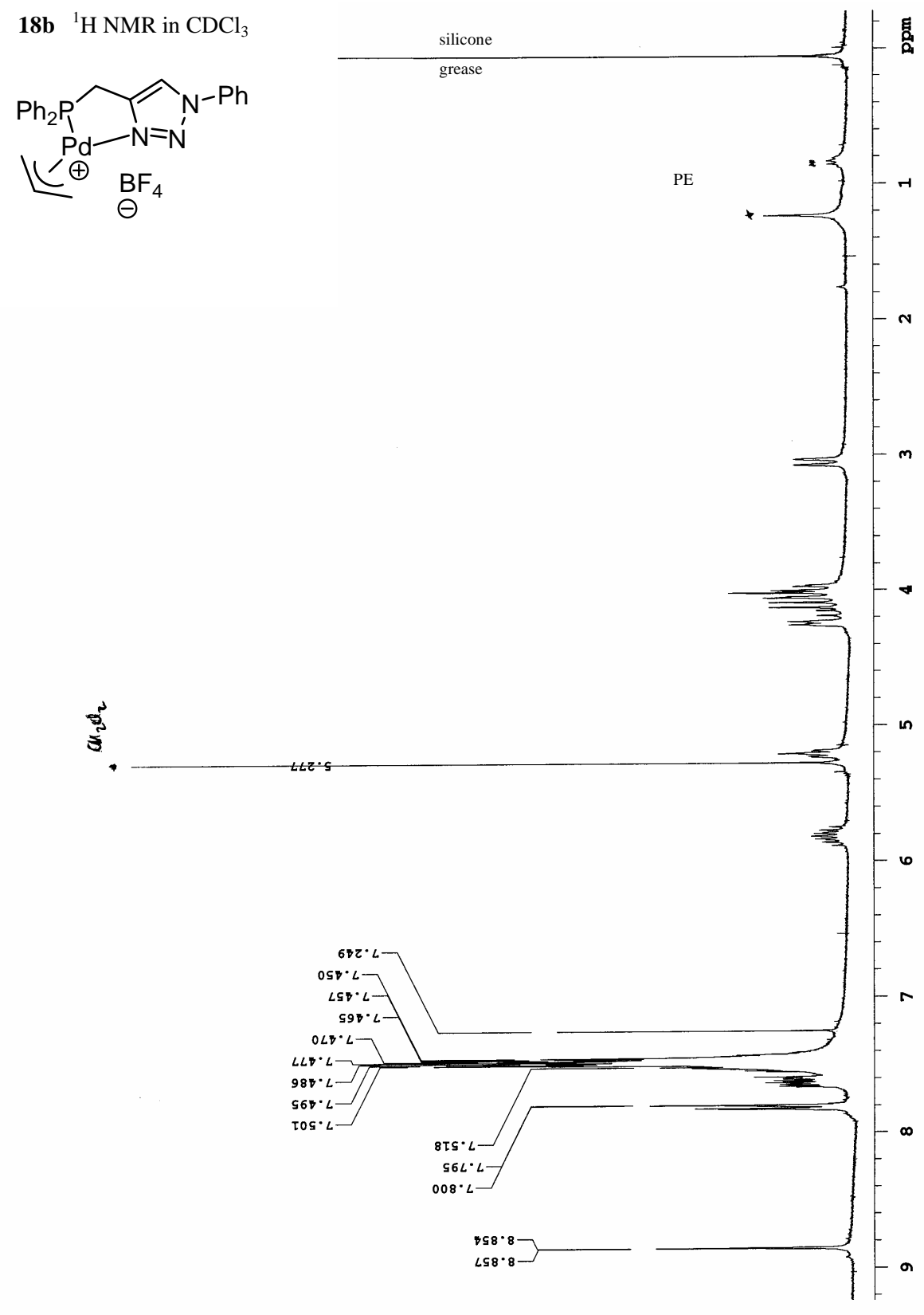



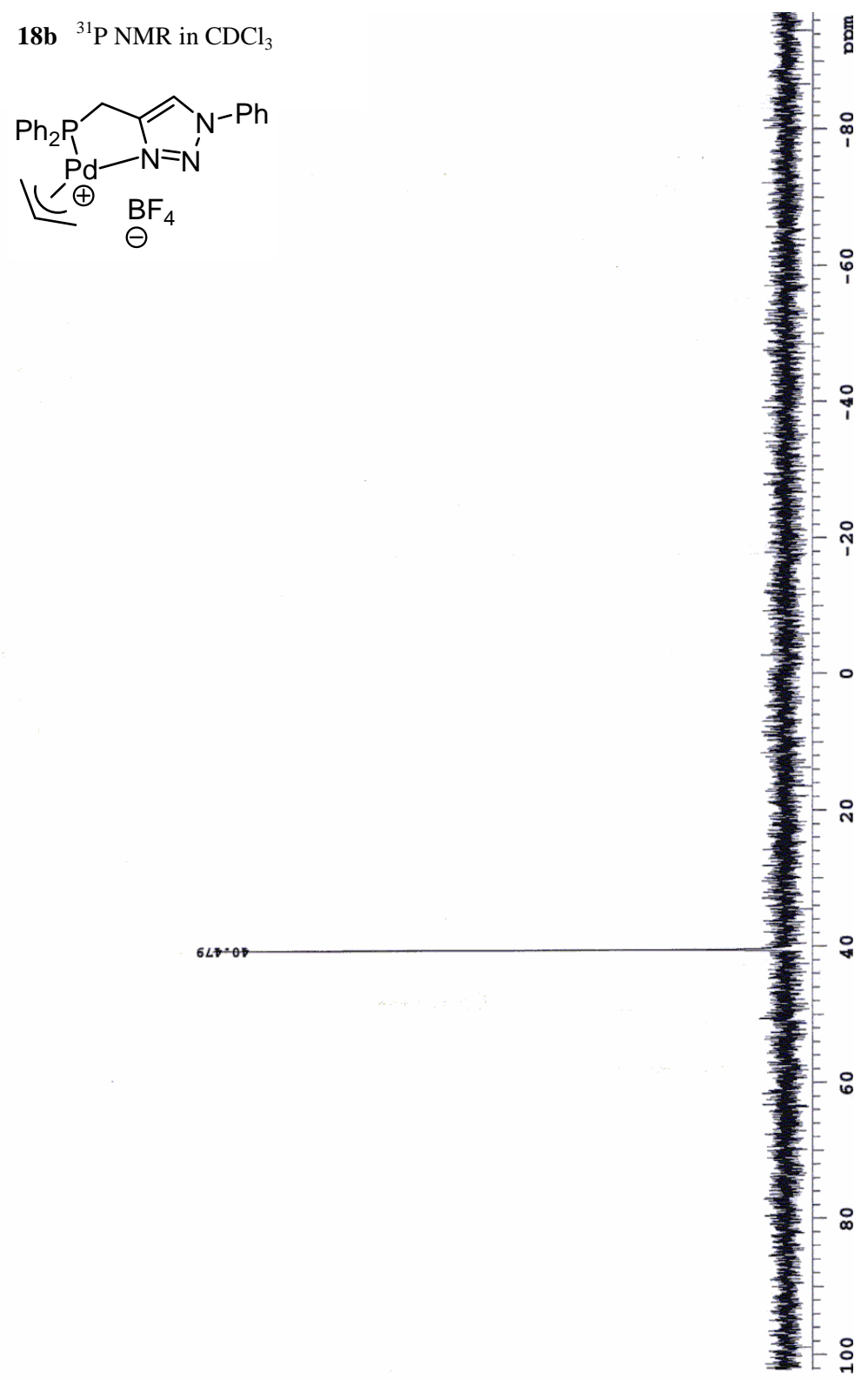

${ }^{1}$ Still,W. C.; Kahn, M.; Mitra, A. J. Org. Chem. 1978, 43, 2923-2925. 